University of Louisville

ThinkIR: The University of Louisville's Institutional Repository

Electronic Theses and Dissertations

8-2014

\title{
Investigation of stabilization mechanisms for colloidal suspension using nanoparticles.
}

Qingwen $\mathrm{He}$

University of Louisville

Follow this and additional works at: https://ir.library.louisville.edu/etd

Part of the Chemical Engineering Commons

\section{Recommended Citation}

He, Qingwen, "Investigation of stabilization mechanisms for colloidal suspension using nanoparticles." (2014). Electronic Theses and Dissertations. Paper 593.

https://doi.org/10.18297/etd/593

This Doctoral Dissertation is brought to you for free and open access by ThinkIR: The University of Louisville's Institutional Repository. It has been accepted for inclusion in Electronic Theses and Dissertations by an authorized administrator of ThinkIR: The University of Louisville's Institutional Repository. This title appears here courtesy of the author, who has retained all other copyrights. For more information, please contact thinkir@louisville.edu. 


\title{
INVESTIGATION OF STABILIZATION MECHANISMS FOR COLLOIDAL SUSPENSION USING NANOPARTICLES
}

\author{
By \\ Qingwen $\mathrm{He}$ \\ B.E., Beijing University of Chemical Tech., 2009
}

A Dissertation Submitted to the J.B. Speed School of Engineering of the University of Louisville in Partial Fulfillment of the Requirements for the Degree of

Doctor of Philosophy

Department of Chemical Engineering

University of Louisville

Louisville, Kentucky

August, 2014 
Copyright 2014 by Qingwen He

All rights reserved 



\title{
INVESTIGATION OF STABILIZATION MECHANISMS FOR COLLOIDAL SUSPENSION USING NANOPARTICLES
}

\author{
By \\ Qingwen $\mathrm{He}$
}

A Dissertation Approved on

July 22, 2014

By the following Dissertation Committee

$\overline{\text { Dr. Gerold A. Willing (Dissertation Director) }}$

Dr. Xiaoan Fu

Dr. Eric Berson

Dr. Cindy K. Harnett 


\section{DEDICATION}

Dedicated to my parents

Mingliang He and Jie Qi

and

Friends 


\section{ACKNOWLEDGEMENTS}

First, I would like to thank my advisor, Professor Gerold A. Willing for helping me pursue my passion in chemical engineering research. He has given freedom at exploring the unknown in research, while providing sufficient guidance to keep me from getting lost. I greatly appreciate his professional training in doing research and academic writing.

In addition to my advisor, I want to express my great gratitude to my dissertation committee members Professor Xiaoan Fu, Professor Eric Berson and Professor Cindy K. Harnett for offering their time and support to help me complete my research project.

Many thanks to Professor Mahendra Sunkara and Thad Druffel for providing me the opportunity of learning the manufacture of dye-sensitized solar cell.

I would also like to thank Ms. Patricia Lumley who has guided me through all the procedures to get my degree.

Lastly, I want to thank my family and friends for being there for me and for having faith in me. 


\title{
ABSTRACT
}

\section{INVESTIGATION OF STABILIZATION MECHANISMS FOR COLLOIDAL SUSPENSION USING}

\section{NANOPARTICLES}

\author{
Qingwen He
}

July 22, 2014

Over the past decade, charged nanoparticles have been found to enhance the stability of colloidal suspensions. One promising explanation of this stabilization mechanism is "Nanoparticle Haloing"- the formation of a non-adsorbing nanoparticle layer surrounding neutral colloids that would induce an effective electrostatic repulsion between them. The objective of this work was to investigate the effect of additional charged nanoparticles on the interaction between neural colloids in nanoparticleregulated complex fluids. Firstly, colloidal probe Atomic Force Microscopy (CP-AFM) is used to directly measure the interaction force in such system with nanoparticle volume fractions varying from $10^{-6}$ to $10^{-2}$. It is found that at a critical low volume fraction $\left(10^{-5}\right)$, the colloidal system is stabilized due to the domination of an electrostatic repulsion between colloidal surfaces induced by the enhanced concentration of nanoparticles in the charged layer. As the nanoparticle concentration is increased, the effective repulsion increases due to a rising charge density built up by the surrounded nanoparticle layer. 
A Debye length fitting model (DLFM) was subsequently developed to theoretically estimate the interaction between colloids in the nanoparticle suspensions. The DLFM suggests: 1) the interaction between microspheres in the presence of nanoparticles is mainly composed of a van der Waals attraction and an electrostatic repulsion; 2) there is a non-zero distance between the nanoparticle layer and the colloidal surface, and the effect of nanoparticle adsorption on the interaction force between colloidal surfaces is negligible at low volume fractions $\left(10^{-6}\right.$ to $\left.10^{-4}\right)$. The follow-up adsorption test and force modeling confirmed that the degree of nanoparticle adsorption is negligible at volume fraction $<0.5 \times 10^{-3}$, but becomes evident as the volume fraction increased to $10^{-3}$, indicating charged nanoparticles are strongly adsorbed onto silica surfaces at relatively high concentrations rather than haloing around them. Thus, we propose that 1) the fundamental mechanism of nanoparticle-regulated stabilization is "nanoparticle haloing" at low nanoparticle concentrations, and becomes "adsorption" at high concentrations; 2) there is a transition region within which the stabilization can be influenced by both nanoparticle haloing and adsorption. This transition was observed around a nanoparticle volume fraction of $10^{-3}$ in our experiments.

Nanoparticle haloing and adsorption two stabilization mechanisms are not mutually exclusive when using charged nanoparticles to regulate the stability of colloidal suspensions; they work continuously over the increasing nanoparticle concentrations. Our study suggests that, when using highly charged nanoparticle to stabilize weakly charged colloidal suspension, the reversibility of stabilization and accessibility of colloidal surfaces can be controlled by simply tuning the nanoparticle concentration. 


\section{TABLE OF CONTENTS}

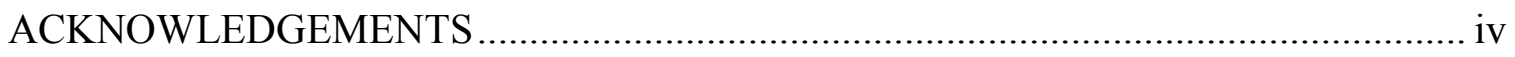

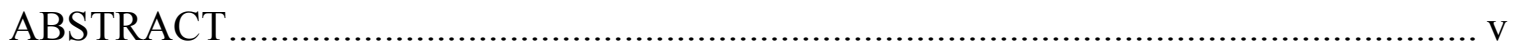

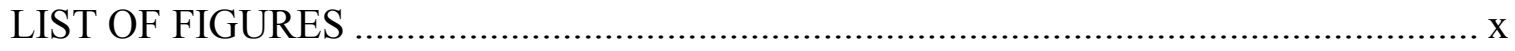

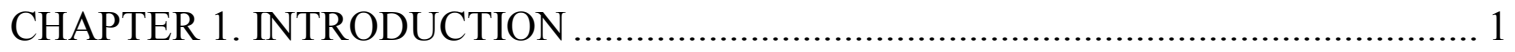

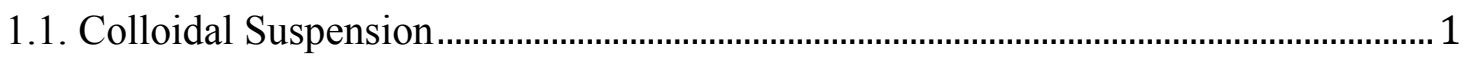

1.2. Interaction Forces between Colloidal Particles ............................................................ 2

1.2.1. van der Waals Forces .......................................................................... 3

1.2.2. Electrical Double Layer Forces ............................................................ 6



1.3. Stabilization of Colloidal Suspensions................................................................... 14

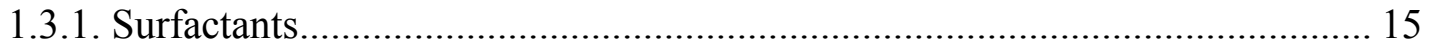

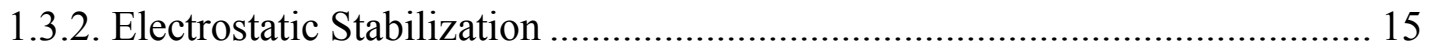

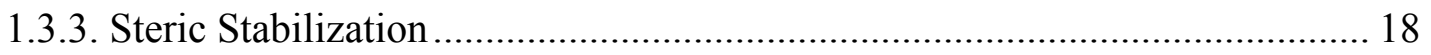

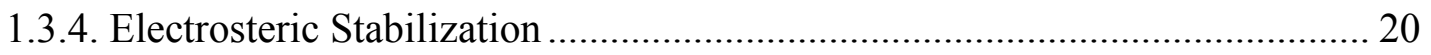

1.3.5. Nanoparticle Haloing - A New Colloidal Stabilization Mechanism ............. 21

1.4. Interaction Measurements among Colloidal Particles .............................................. 24

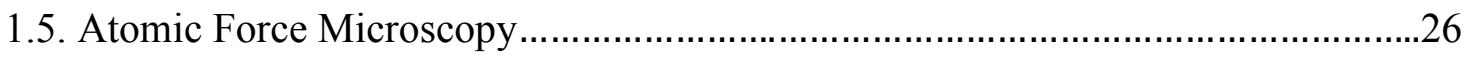


1.5.1. Working mode

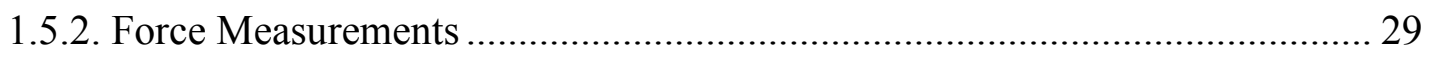

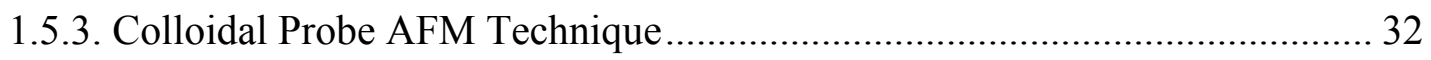

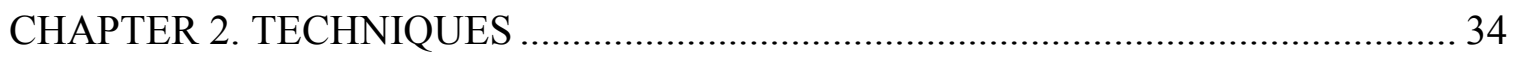

2.1. Force Measurements by Atomic Force Microscopy …............................................... 34

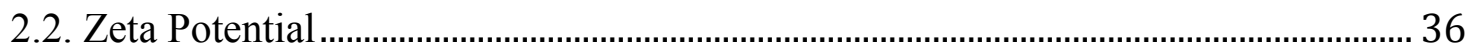



CHAPTER 3. FORCE MEASUREMENTS BETWEEN COLLOIDAL SURFACES IN





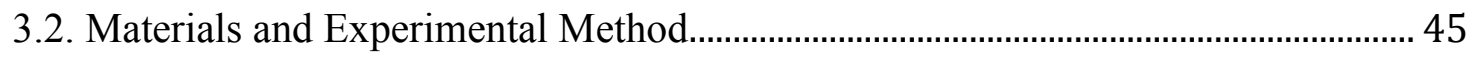

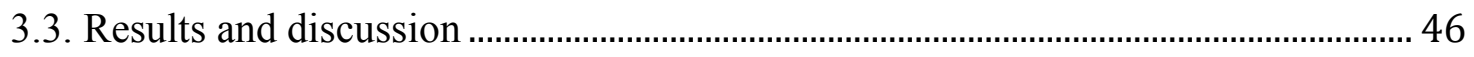

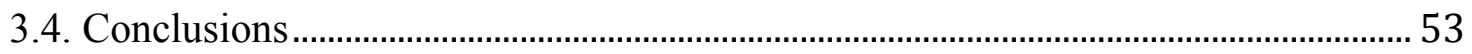

CHAPTER 4. MODELLING THE STABILIZATION MECHANISM OF COLLOIDAL SUSPENSION USING HIGHLY CHARGED NANOPARTICLES .............................. 54

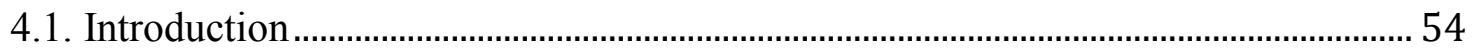

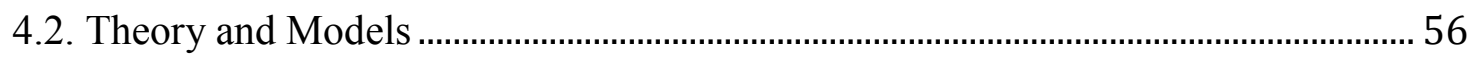

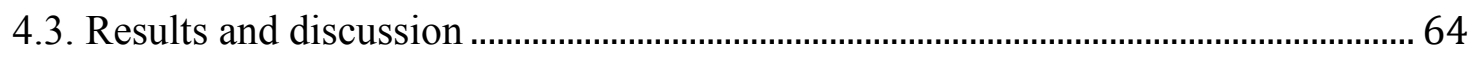

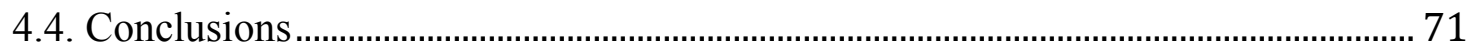


CHAPTER 5. TUNING THE STABILZATION MECHANISM OF NANOPARTICLE-

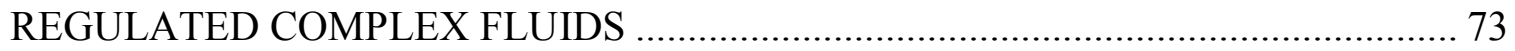

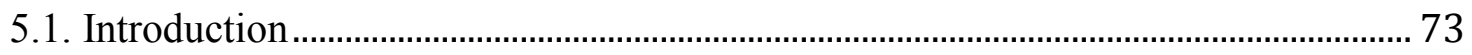

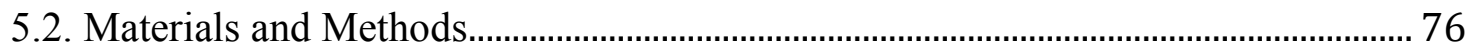

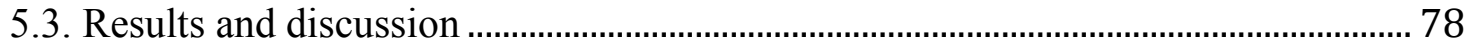

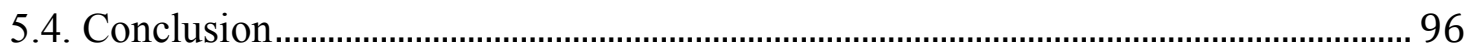

CHAPTER 6. CONCLUSIONS AND FUTURE DIRECTIONS ................................ 98

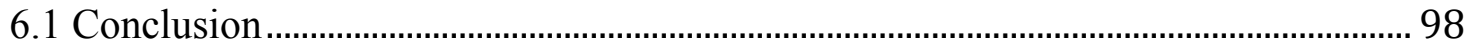

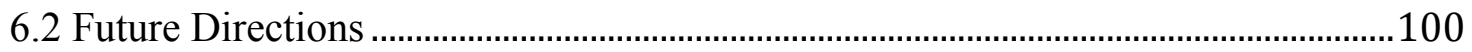

6.2.1 Determine the Critical Conditions of Nanoparticle Halo and Adsorption..... 100

6.2.2 Introduce nanoparticle halo mechanism to industry process ...................... 100

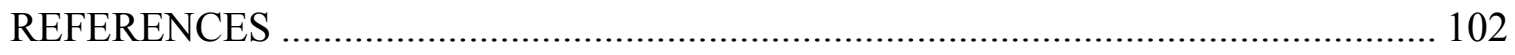

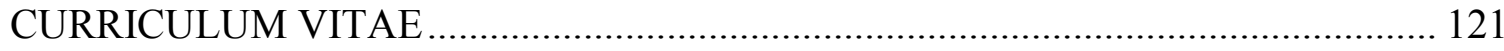




\section{LIST OF FIGURES}

Figure 1.1 a) Gouy-Chapman double layer model. b) Stern model of double layer ........... 8 Figure 1.2 Scheme of the DLVO theory: (a) Surfaces repel strongly, small colloidal particles remain stable; (b) Surfaces are at equilibrium at secondary minimum if it is deep enough, colloids remain kinetically stable; (c) Surfaces come into secondary minimum, colloids coagulate slowly; (d) The critical coagulation concentration, surfaces may remain in secondary minimum or adhere, colloids coagulate rapidly; (e) Surfaces and

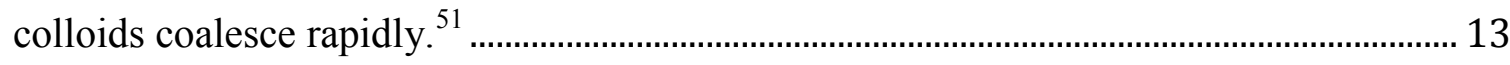

Figure 1.3 Schematic illustration of electrostatic stabilization.......................................... 18

Figure 1.4 Schematic illustration of steric stabilization.................................................... 19

Figure 1.5 Schematic illustration of electrostatic stabilization........................................... 21

Figure 1.6 Schematic illustration of nanoparticle-halo stabilization.................................. 23

Figure 1.7 Schematic of an atomic force microscope....................................................... 26

Figure 1.8 Schematic of a typical cantilever deflection-vs.-z scanner height curve......... 31

Figure 1.9 SEM images of a colloid probe: a silica sphere $(100 \mathrm{~nm})$ attached to the apex

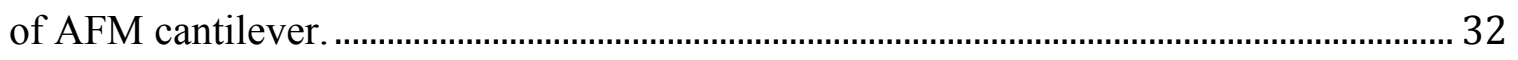

Figure 2.1 A representation of the XE100 AFM used during experiments. ${ }^{141}$.................. 34

Figure 2.2 Schematic description of AFM force measurement for a) sphere-sphere

system, b) sphere-plate system. .35 
Figure 2.3 Schematic of typical Piezo Scanner Deflection-vs.-Piezo Height curve and corresponding force-vs.-distance curve. 36

Figure 2.4 The double layer of a plane. 37

Figure 3.1 Semi-log plot of effective zeta-potential $(\xi)$ of the binary composite sphere as a function of zirconia nanoparticle volume fraction in $\mathrm{pH} 1.5$ solution $\left(\Phi_{\text {silica }}=10^{-}\right.$ ${ }^{2} ; \Phi_{\text {zirconia }}=10^{-6}-10^{-2}$, silica diameter $=1 \mu \mathrm{m}$, nano diameter $\left.=8 \mathrm{~nm}\right)$. 48

Figure 3.2 Experimental force curve between a silica microsphere and plate without nanoparticles at $\mathrm{pH}=1.5$; Theoretical van der Waals force vs. separation between two silica colloidal spheres.

Figure 3.3 Experimental interaction forces between a silica microsphere and plate in different zirconia nanoparticle suspensions at size ratios of A) 100 B) 60 C) 20, and D) 10 52

Figure 4.1 The potential profile between planar double layers 57

Figure 4.2 Comparison of electrostatic interaction between a sphere and flat based on theoretical integration and HHF approximation at $\mathrm{pH} 1.5$ and zeta potential of a) $20 \mathrm{mv}$, b) $30 \mathrm{mv}$, c) $40 \mathrm{mv}$ and d) $50 \mathrm{mv}$. 61

Figure 4.3 Experimental interaction forces between a silica microsphere and plate in different zirconia nanoparticle suspensions at size ratios of A) 100 B) 60 C) 20, and D) 10 65

Figure 4.4 Plots of force vs. separation measured by AFM in nanoparticle suspensions with varying volume fraction (blue circles) at $\mathrm{pH}=1.5$, and the theoretical force curves calculated by the fitting model using an appropriate effective Debye length (red solid lines). Size ratios: A) 100, B) 60, C) 20. 67 
Figure 4.5 Schematic description of the effect of the nanoparticle volume fraction on the gap between silica sphere and zirconia nanoparticle. 70

Figure 5.1 Force profiles between a silica microsphere and plate in varying volume fractions of zirconia nanoparticles at size ratio of (a) 100; (b) 60. 79

Figure 5.2 Experimental force profiles and fitting results by using DLFM at size ratio of (a) 100; (b) 60. The solid lines represent the total interaction force calculated by the DLFM. 82

Figure 5.3 The predicted pair potential between a nanoparticle and microsphere. 84

Figure 5.4 SEM image obtained on silica plate that had been immersed in a volume fraction $10^{-4}$ nanoparticle solution for $30 \mathrm{~min}$ at $\mathrm{pH} 1.5$. Zirconia nanoparticles are highlighted in red by ImageJ as seen in b; nanoparticle number and adsorption fraction is estimated by this program as well. 85

Figure 5.5 Approximate surface coverage fraction $(\theta)$ as the function of nanoparticle concentration at $\mathrm{pH} 1.5$. 86 Figure 5.6 (a) Curve fitting of Volume fraction/ surface courage Vs. Volume fraction to Langmuir isotherm; (b) Original form of Langmuir isotherm. 88

Figure 5.7 Schematic diagram of the interactions between a probe sphere and a flat surface induced by a) nanoparticle halo; b) adsorption. 90

Figure 5.8 Force curves calculated at different thickness of adsorb layer. 91

Figure 5.9 Force curves measured at volume fraction $10^{-2}$ and $10^{-4}$, and fitting results using the modified DLFM at size ratio of a) 100 ; b) 60 . Sold line presents modified DLFM fitting curve; dash line stands for the original DLFM fitting curve. 93 
Figure 5.10 Fitted Debye length as the function of nanoparticle volume fraction at size

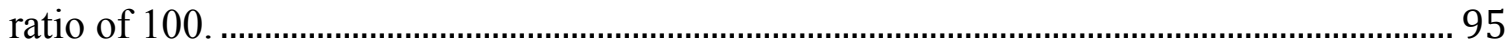




\section{CHAPTER 1}

\section{INTRODUCTION}

\subsection{Colloidal Suspension}

A colloid is defined as a microscopic material dispersed throughout a continuous medium with a dispersed-phase diameter of between approximately 1 and $1000 \mathrm{~nm}$. Colloidal dispersions are very common in daily life, as seen in such forms as foams (e.g. whipped cream), emulsions (e.g. milk), and sols (e.g. pigmented ink). Depending upon the state of the dispersed phase and the dispersion medium, eight different types of colloidal dispersions can be identified (Table 1.1).

Table 1.1. Different types of colloidal dispersions

\begin{tabular}{|c|c|c|c|}
\hline \multirow{2}{*}{ Dispersed phase } & Solid & Liquid & Gas \\
\cline { 2 - 4 } & Solid sol or solid & Sol or colloidal \\
suspension & suspension & Solid aerosol \\
\hline \multirow{2}{*}{ Solid } & Gel & Emulsion & Liquid aerosol \\
\hline Liquid & Solid foam & Foam & \\
\hline
\end{tabular}

A key feature of colloidal systems is that the contact area between particles and

dispersing medium is large, leading to the suspension behavior being significantly affected by interparticle interactions. Thus, the control of these interparticle interactions 
in colloids gives rise to rich phase behaviors which can be widely applied to numerous application areas, including drug delivery, ceramic processing, and coatings. ${ }^{1-10}$

Colloidal processing provides the potential to reliably produce microstructure materials (i.e. ceramic films) through careful control of initial suspension conditions and their evolution during fabrication. ${ }^{11-13}$ This approach has five basic steps: (1) powder synthesis, (2) suspension preparation, (3) consolidation into the desired component shape, (4) removal of the solvent phase, and (5) densification to obtain the final microstructure required for optimal performance. Due to the persistence of defects introduced in any stage of the fabrication process there is a continual drive toward better understanding of colloidal stability and assembly to achieve the desired distribution of phases.

Interaction forces between colloidal particles in all suspensions play an important role in determining the properties of the materials, such as the shelf life, stability, and rheology, the behavior of a number of industrial processes (e.g. mixings, membrane filtrations). This arises due to the dependence of the behavior of the suspension on the magnitude and range of the surface interactions. ${ }^{14}$ For instance, the surface charge properties, the dispersing medium and the subsequent collision efficiency between particles have been shown to significantly influence the stability and the rheology of particulate suspension. ${ }^{15}$ There have been well-developed theories that describe the interparticle interactions in colloidal suspensions, most of which can be resolved either analytically or numerically in terms of the underlying fundamentals.

\subsection{Interaction Forces between Colloidal Particles}

Through careful control of interparticle forces, colloidal suspensions can be prepared in the dispersed, weakly flocculated or strongly flocculated states. In the 1940s, 
Derjaguin-Landau and Verwey-Overbeek had developed a theory to account for the rates at which colloidal particles in aqueous solution can be expected to undergo sticking collisions, forming agglomerates, and eventually precipitating out of suspension. This socalled DLVO theory provides a basic framework for study of colloidal interactions.

The DLVO theory ${ }^{16,17}$ is built on the assumption that the forces between two surfaces in a liquid can be regarded as the sum of two contributions. These are the London-van der Waals forces and the electric double layer forces due to the electromagnetic effects of the molecules within the particles and the overlapping of the electrical double layers of two neighboring particles. For two identical particles the former is always attractive and the latter is always repulsive. The fact that colloidal particles in a liquid medium tend to form persistent aggregates through collisions caused by Brownian motion implies an inter-particle attractive force (van der Waals force). Three distinct types of forces contribute to the total long-range attractive interaction between polar molecules: these are the induction force, the orientation force and the dispersion force. Cases in which van der Waals forces alone determine the total interaction are limited to "simple systems", for instance, to interactions in a vacuum, nonpolar wetting films on surfaces, and interaction of particles in a non-polar media (oils). In aqueous electrolyte solutions long-range electrical double layer forces also occurred. The interplay between these two interactions has many important consequences. For instance, clay particles and silt carried by rivers coagulate upon coming across the high salt concentration of the sea to form extensive deltas. Electrostatic forces are also crucial in the behavior of biological systems.

\subsection{1. van der Waals Forces}


Many methods have been reported in the literature to calculate the London-van der Waals interaction energy ${ }^{18-21}$ In general these can be separated into two separate approaches to calculate the van der Waals forces between surfaces: the microscopic and the macroscopic.

In the microscopic approach, London ${ }^{22}$ and $\mathrm{Wang}^{23}$ gave a quantum-mechanical analysis of the force between a pair of non-polar molecules in that the perturbation theory was used to solve the Schrödinger equation for two hydrogen atoms at large separation, including the interactions between the electrons and protons of the two atoms. Afterwards, a more detailed analysis of the interactions had been done by taking higher moments into account $^{24}$, and the effect of retardation when the distance of separation between the molecules exceeds the characteristic wavelength of radiation emitted due to dipolar transitions $^{25}$. Subsequently, Hamaker ${ }^{26}$ and de $\mathrm{Boer}^{27}$ investigated theoretically the dispersion forces acting between colloidal objects. They considered spherical bodies, assumed pairwise additivity of interatomic dispersion energies, and demonstrated the essential results that although the range of atomic forces was of the order of atomic dimensions, the sum of the dispersion energies resulted in an interaction range for colloidal bodies of the order of their dimensions. Like most simple theories the Hamaker approach to interactions has the advantage of not only ease in understanding, but also in application over a wide range.

For two spheres of equal radius, $a$, at a surface to surface separation distance, $D$, apart along the center to center axis, the total interaction energy, $V_{\mathrm{A}}$, is given by

$$
V_{A}(D)=-\frac{A}{6}\left[\frac{2 a^{2}}{D^{2}+4 a D}+\frac{2 a^{2}}{(D+2 a)^{2}}+\ln \left(1-\frac{4 a^{2}}{(D+2 a)^{2}}\right)\right.
$$


The quantity $A$ is called the Hamaker constant. If the Hamaker constant, $A$, is known, it is possible to calculate the interaction energy between the particles provided that the particle radius, $a$, and interparticle distance, $D$, are known.

In the case of the interaction between a sphere and a plane, the total energy can be obtained by letting one of the radii go to infinity. The result is

$$
V_{A}(D)=-\frac{A}{6}\left[\frac{a}{D}+\frac{a}{D+2 a}+\ln \left(\frac{D}{D+a}\right)\right]
$$

where $a$ is the sphere's radius, and $D$ the distance from the sphere surface to the plane. The above formulae for the interaction energy between colloidal bodies are based on the assumption that the interaction is pairwise additive; the influence of neighboring atoms on the interaction between any pair of atoms is ignored. In gaseous media these effects are small, and the assumptions of pairwise additivity can hold, but this is not the case for condensed media such as liquid. Furthermore, the additivity approach cannot be readily extended to bodies interacting in a medium.

In the macroscopic approach, the problem of additivity is completely avoided in the Lifshitz theory ${ }^{28}$ where atomic structure is neglected and large bodies are treated as continuous media and forces are derived in terms of the bulk properties such as dielectric constants and refractive indices. However, it should be pointed out that all the equations mentioned above for the interaction energies remain valid even within the framework of continuum theories. Only the Hamaker constant is to be calculated in a different way. To calculate the Hamaker constant, the knowledge of the dielectric spectra over the entire frequency range for all of the individual materials comprising the system is required. ${ }^{29-33}$ The attractive force between two colloidal objects can thus be calculated using the interaction energy expression as 


$$
F_{A}=-\frac{d V_{A}}{d D}
$$

\subsubsection{Electrical Double Layer Forces}

As noted before the van der Waals force between the same particles in a liquid is always attractive, if this is the only operating force, all dispersed particles may aggregate together and precipitate out of suspension as a solid cake. Fortunately this is not the case as particles in water or any liquid of high dielectric constant are usually charged. Aggregation can be prevented from occurring as the long-range repulsive forces will prevail over the van der Waals attractive forces.

\section{Electrical Double Layer Around Particle}

It can be concluded according to what was observed in colloidal systems that particles dispersed in water and any liquid of high dielectric constant usually develop a surface charge. The charging of a surface in a liquid can be brought about in two charging mechanisms ${ }^{14}$ :

(1) By the ionization or dissociation of surface groups, which leaves behind a charged surface (e.g., the dissociation of protons from carboxylic groups, which leaves behind a negatively charged surface) and

(2) By the adsorption (binding) of ions from solution onto a previously uncharged surface. The adsorption of ions from solution can also occur onto oppositely charged sites, also known as ion exchange.

Since the system as a whole is electrically neutral, the dispersing medium must contain an equivalent charge of the opposite sign. These charges are carried by ions, i.e., by an excess of ions of one sign on the particle surface and an excess of ions of the opposite sign in the solution. Hence, if we consider an individual particle immersed in the 
liquid, it is surrounded by an electric double layer. One of this double layer is formed by the charge on the surface of the particles. Another layer of the electrical double layer is formed by the excess of oppositely charged ions in the solution. As a result of their thermal motion the electric charge carried by this layer extends over a certain distance from the particle surface, and dies out gradually with increasing distance (diffuse layer) into the bulk liquid phase.

\section{Distribution of Electrical Charge and Potential in Double Layer}

The first approximate theory for the electrical double layer was given by Gouy, Chapman, and Debye and Hückel ${ }^{17}$. In this theory the average charge distribution and the corresponding electrical potential function have been related on the basis of the PoissonBoltzmann equation $(\mathrm{PBE})^{34}$ :

$$
\nabla^{2} \psi=\frac{-1}{\varepsilon_{0} \varepsilon} \sum_{i} n_{i}^{0} z_{i} e \exp \left(\frac{-z_{i} e \psi}{k T}\right)
$$

where $\psi$ is the electrical potential, $n_{i}^{0}$ the number density of ions of valency $z_{i}, k$ the Boltzmann constant, $T$ the absolute temperature, $\varepsilon_{0}$ the permittivity of vacuum, $\varepsilon$ the dielectric constant of component $i$ and $e$ the elementary charge.

The above PBE has been deduced using a number of simplifying assumptions such as the electrolyte is an ideal solution with uniform dielectric properties, the ions are point charges, and the potential of the mean force and the average electrostatic potential are identical. Besides, the PBE is only applicable to the system with a symmetrical electrolyte or a mixture of electrolytes of the same valency type. According to this theory, the average charge density at a given point can be calculated from the average value of the electrical potential at the same point with Boltzmann's theorem. The electrical potential distribution can be related to the charge density with the aid of Poisson's 
equation. As a matter of fact, the Gouy-Chapman theory has a rather serious defect, which is mainly a consequence of neglecting the finite dimensions of the ions. In dilute solutions, where the extension of the diffuse layer is considerable, this neglect is to some degree permissible; but in more concentrated electrolyte solutions the picture in terms of the Gouy-Chapman model becomes incorrect in essential details.
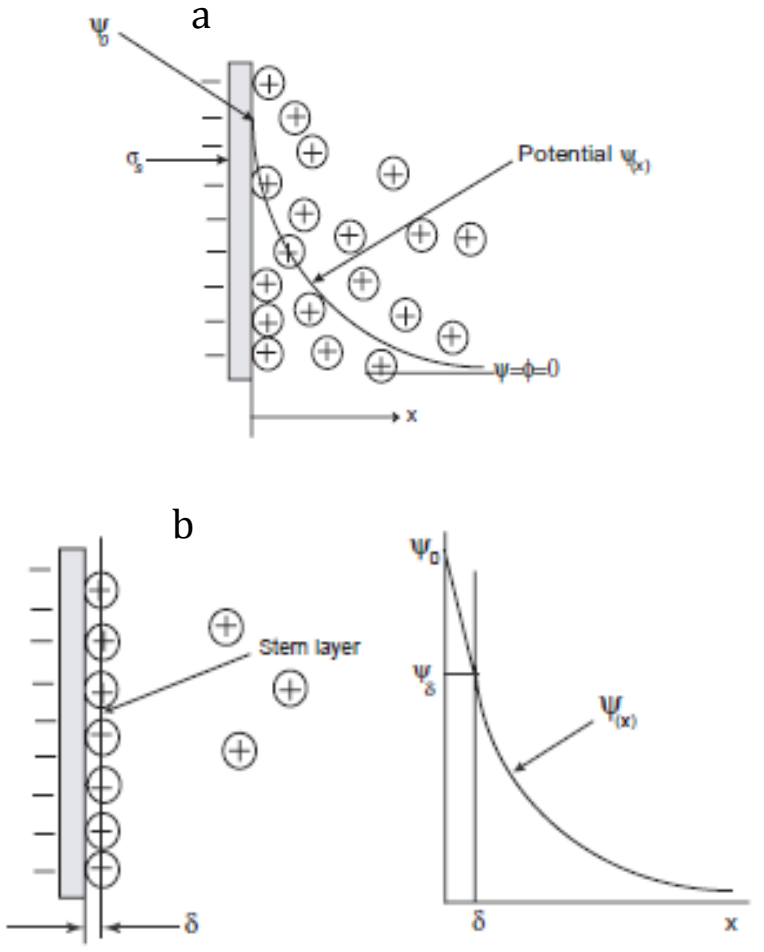

Figure 1.1 a) Gouy-Chapman double layer model. b) Stern model of double layer Stern ${ }^{35}$ has modified the Gouy-Chapman model by taking into consideration the finite size of real ions, underlying the double layer theory for a solid wall by dividing the charges within liquid into two parts (Figure 1.1b). One part is considered as a layer of ions adsorbed to the wall, and is represented in the theory by a surface charge concentrated in a plane at a small distance $\delta$ from the surface charge on the wall, also known as the outer Helmholtz plane (OHP).The second part of the liquid charge is then 
taken to be a diffuse space charge, as in the old theory, extending from the OHP at $x=\delta$ to infinity where the PBE can apply.

The non-linear PBE is used to calculate the potential distribution inside the diffusive part of the electric double layer between two surfaces ${ }^{15,34}$. According to the non-linear PBE the aqueous solution is defined by its static dielectric constant only. The surface charge is usually taken as averaged over the surface and the discrete nature of ions is not considered.

In order to calculate the potential distribution around a particle, not only is the PBE needed but the boundary conditions have to be specified. A choice of boundary conditions is available at the particle surface. It is important to choose physically meaningful conditions at the particle surfaces, which depend on the colloidal material being considered. For metal sols in a solution, a constant surface potential boundary condition is appropriate; whereas a constant surface charge boundary condition may be appropriate when the surface charge is caused by crystal lattice defects, such as in clay minerals. In the case of biomaterials and oxide surfaces, the charge can be generated by surface dissociation reaction that is influenced by the solution conditions. This can be described by a boundary condition known as charge regulation ${ }^{14}$.

\section{Interaction Force between Double Layers}

When two like-charged particles approach each other, their electrical double layers will start to overlap, resulting in a repulsive force that opposes further approach. For dilute systems where just two particles can be considered in the interaction, it is possible to obtain analytical expressions for the calculation of the repulsive interaction energy between two spherical particles on the basis of the interaction energy equations 
derived for infinite flat plates of the same material with either the Derjaguin approximation ${ }^{36}$ or the linear superposition approximation (LSA) ${ }^{37}$ as below:

$$
V_{R}=\frac{128 \pi a_{1} a_{1} n_{\infty} k T}{\left(a_{1}+a_{2}\right) \kappa^{2}} \gamma_{1} \gamma_{1} \exp (-\kappa h)
$$

where $h$ is the surface-surface separation between the particles, $a$ the particle radius of different sizes, $\kappa$ the Debye-Hückel reciprocal length, $n_{\infty}$ the bulk density of ions and $\gamma$ the reduced surface potential expressed as

$$
\gamma=\tanh \left(\frac{z e \psi}{4 k T}\right)
$$

The above equation is only valid when both the conditions $\kappa a>5$ and $h \ll a$ are satisfied. Debye length is the measure of a charge carrier's net electrostatic effect in solution, and how far those electrostatic effects persist. There are many other expressions available based on various assumptions for sphere-sphere double layer interaction energy ${ }^{37-32}$. In general, the LSA method yields the correct interaction at large separations for all surface potentials and particle sizes; Derjaguin's integration gives accurate results for large particles at short distances; and the McCartney and Levine formulation ${ }^{43}$ is a good approximation at all separations but small potentials. It should be noted that although the first two methods themselves place no restriction on the potentials, the resulting expressions often do because of the difficulty in solving the PBE. Therefore, care must be taken in choosing the right expression.

In the case of concentrated colloidal dispersions, however, interaction energy between particles (as in a gel layer) is multiparticle in nature so modification of the two body interaction has to be made in order to allow for multiparticle interactions. A method by which the multiparticle nature of such interactions can be taken into account is to use a cell model ${ }^{44}$ combined with a numerical solution of the non-linear PBE in spherical co- 
ordinates $^{45-49}$. This cell model is based on the Wigner and Seitz cell model ${ }^{50}$ that approximated the free electron energy of a crystal lattice by calculating the energy of a single crystal since it had the same symmetry as the lattice.

The concentrated colloidal dispersion can now be considered as being divided into spherical cells so that each cell contains a single particle and a concentric spherical shell of an electrolyte solution, having an outer radius of certain magnitude such that the particle cell volume ratio in the unit cell is equal to the particle volume fraction throughout the entire suspension, and the overall charge density within the cell is zero (electro-neutral). This kind of approach gives a mean field approximation that accounts for multiparticle interactions to yield the configurational electrostatic free energy per particle $^{47}$. By equating the configurational free energy with the pairwise summation of forces in hexagonal arrays, an expression for the repulsive force between two particles can be obtained which implicitly takes into account the multiparticle effect ${ }^{45}$

$$
F_{R}(D)=\frac{1}{3} S_{\beta}(D) n^{0} k T\left(\cosh \left(\frac{z e \psi_{\beta}(D)}{k T}\right)-1\right)
$$

where $S_{\beta}(D)$ is the surface area of the spherical cell around the particle, $n^{0}$ the ion number concentration, $k$ the Boltzmann's constant, $T$ the absolute temperature, $z$ the valence of the ions, $e$ the elementary electronic charge and $\psi_{\beta}(D)$ the potential at the surface of the spherical cell.

In order to evaluate the above equation the size of the cell and the potential at the cell surface need to be known. The radius of the fluid shell can be determined with the volume fraction approach ${ }^{47}$. The potential at the outer boundary of the cell may be determined by solving the non-linear PBE in spherical co-ordinates numerically.

\subsubsection{DLVO Theory}


The DLVO theory is named after Derjaguin and Landau ${ }^{16}$, Verwey and Overbeek $^{17}$ who developed it in the 1940s. The theory describes the force between charged surfaces interacting through a liquid medium. It combines the effects of the London-van der Waals attraction and the electrostatic repulsion due to the overlap of the double layer of counterions. The central concept of the DLVO theory is that the total interaction energy of two surfaces or particles is given by the summation of the attractive and repulsive contributions. This can be written as

$$
V_{\text {total }}=V_{A}+V_{R}
$$

where the total interaction energy $V_{\text {total }}$ is expressed in terms of the repulsive double layer interaction energy, $V_{\mathrm{R}}$, and the attractive London-van der Waals energy, $V_{\mathrm{A}}$. Contrary to the double layer interaction, the van der Waals interaction energy is mostly insensitive to variations in electrolyte strength and $\mathrm{pH}$. Additionally, the van der Waals attraction must always be greater than the double layer repulsion at extremely small distances since the interaction energy satisfies a power-law, whereas the double layer interaction energy remains finite or increases far more slowly within the same separation range.

According to DLVO theory, the phase behavior of a colloidal system is determined by competition between the van der Waals attraction and electrostatic repulsion. Different DLVOinteractions are summarized in Figure 1.2. Depending on the surface charge density and electrolyte concentration, different phase behaviors may occur: 


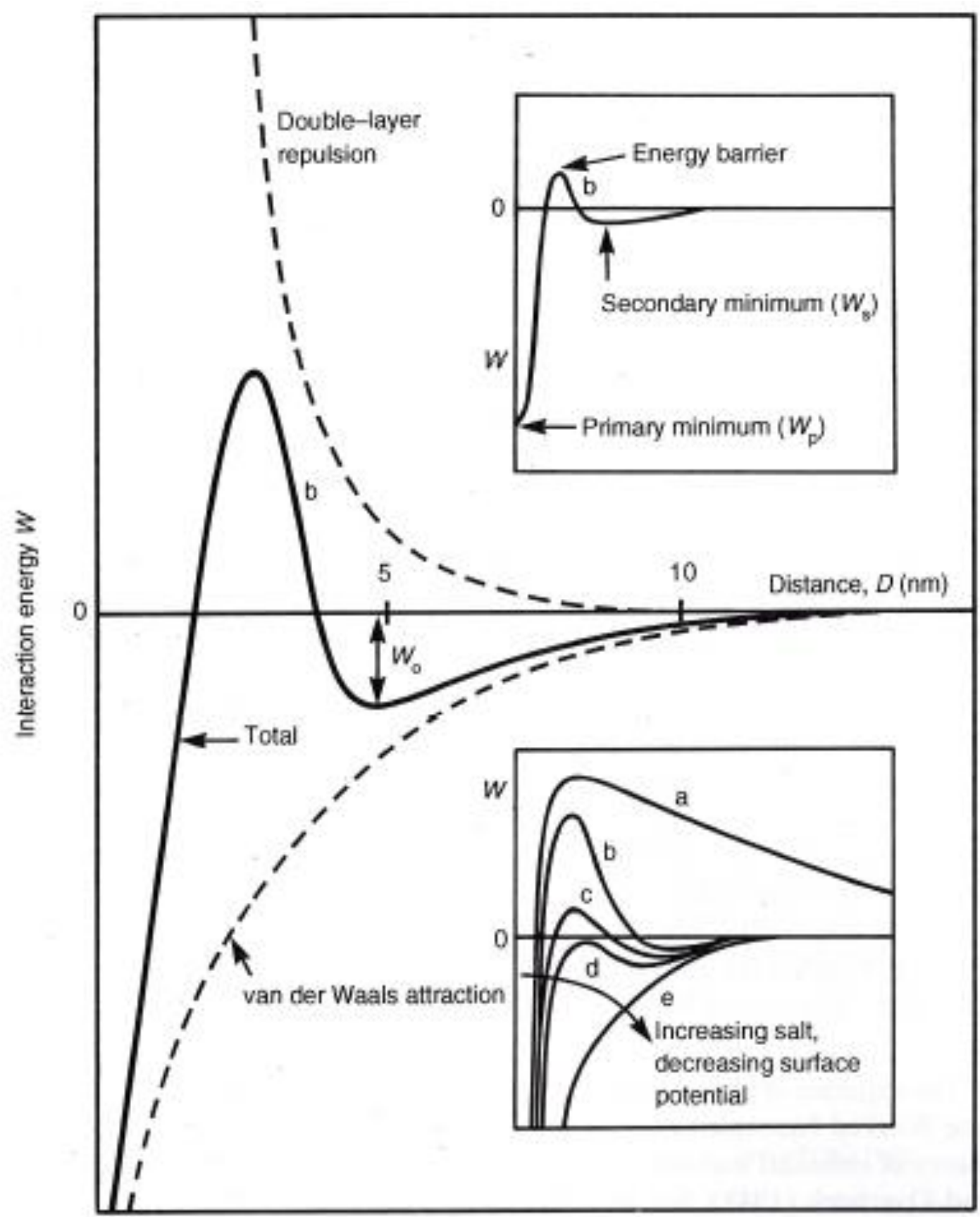

Figure 1.2 Scheme of the DLVO theory: (a) Surfaces repel strongly, small colloidal particles remain stable; (b) Surfaces are at equilibrium at secondary minimum if it is deep enough, colloids remain kinetically stable; (c) Surfaces come into secondary minimum, colloids coagulate slowly; (d) The critical coagulation concentration, surfaces may 
remain in secondary minimum or adhere, colloids coagulate rapidly; (e) Surfaces and colloids coalesce rapidly. ${ }^{51}$

(1) For highly charged surfaces in dilute electrolyte, a strong repulsion is obtained so that the colloidal particles remain stable in this condition (curve a). (2) In higher electrolyte solutions, a secondary minimum appears at curve b. Even though the thermodynamic equilibrium state may be with the particles in contact in the deep primary minimum, the energy barrier may be too high for the particles to overcome during any reasonable time period. As a result, the particles will either come to the weaker secondary minimum well or stay totally dispersed in solution (curve b). (3) At low surface charge density or potential, the energy barrier is relatively low, leading to a slow aggregation among colloidal particles (curve c). Once the energy barrier falls below zero, the particles coagulate rapidly (curve d). The colloidal system becomes unstable. (4) In the absent of a surface charge or potential, the interaction is dominated by the van der Waals attraction. Two surfaces strongly attract each other at all separations (curve e). ${ }^{51}$

\subsection{Stabilization of Colloidal Suspensions}

Particles in suspension tend to aggregate especially for large sizes due to the effect of gravity. Stability refers to the condition in which the colloidal particles do not aggregate at a significant rate. The DLVO theory suggests that the stability of a colloidal suspension is determined by the sum of the van der Waals attraction and repulsion between colloidal particles as they approach each other driven by the Brownian motion. When the van der Waals attraction is stronger than the repulsion, colloidal particles will aggregate, and the state of the suspension is unstable. If the repulsion is sufficient high to overcome the van der Waals force, the system will achieve stability. Thus, in order to 
enhance the stability of a colloidal suspension, the repulsion between the particles needs to be strengthened. According to the types of repulsion, the typical fundamental mechanisms of colloidal stability are divided into two kinds: steric repulsion, and electrostatic repulsion. In practice, the most popular way to achieve this is to add an additional component like a surfactant or polymer that adsorbs on the colloidal particles and changes their surface properties. ${ }^{52}$

\subsubsection{Surfactants}

When added to an aqueous solution, surfactants adsorb readily onto the surface of a hydrophobic particle as well as hydrophilic surfaces in an apolar medium. They selfassemble on the surface to form a monomolecular film which typically eliminates the charges on the particle. The repulsive force between colloidal particles is mainly determined by the interaction between the surfactant monolayers. In an apolar solution, the apolar chains of the surfactant are exposed to the solution and keep colloidal particles apart like a short brush; for charged surfactants, the long-range electrostatic repulsion will operate effectively due to the reduction of the van der Waals attraction. ${ }^{49}$

Although surfactants provide an efficient way to enhance the stability of colloidal suspensions, they has some disadvantages. For example, the surfactant may contaminate the heat transfer media. Surfactants may produce foams upon heating, when heating and cooling are routine processes in heat exchange systems. The attached surfactants on colloidal surfaces may also change the thermal properties of the colloidal particles and solvent. $^{49}$

\subsubsection{Electrostatic Stabilization}


Another effective way to counterbalance the van der Waals attraction between colloidal particles in polar liquids is to shell the particles with a Coulombic repulsion. In liquid dispersion media, ionic groups can adsorb to the surface of a colloidal particle through different mechanisms to form a charge layer. To maintain electro-neutrality, an equal number of counterions with the opposite charge will surround the colloidal particles and give rise to overall charge-neutral double layers. In charge stabilization, it is the mutual repulsion of these double layers surrounding particles that provides stability. Thus, if the electric potential associated with the double layer is sufficiently high, the electrostatic repulsion between the particles prevents their aggregation (Figure 1.3). In an electrostatic-regulated system, the degree of dispersion is controlled by adjusting either ionic strength or $\mathrm{pH}$ of the electrolyte solution. There has been several analytical approximations or numerical solutions for calculation of the electrostatic potential energy. ${ }^{36-47}$ For spherical particles of equal size that approach one another under conditions of constant potential, the electrostatic double-layer interactions $V_{\text {electro }}$ can be estimated by means of the well-known Hogg-Healy-Fuerstenau (HHF) formula ${ }^{39}$, given by

$$
V_{\text {electro }}=2 \pi R \varepsilon_{0} \varepsilon_{r} \psi_{0}^{2} \ln [1+\exp (-\kappa D)]
$$

provided $\kappa a$ is sufficiently large $(>10)$. In contrast, when the double layer around each particle is extensive $(\kappa a<5), V_{\text {electron }}$ is given by ${ }^{15,52}$

$$
V_{\text {electro }}=\pi R \varepsilon_{0} \varepsilon_{r} \psi_{0}^{2} \exp (-\kappa D)
$$

where $\varepsilon_{0}$ is the vacuum permittivity, $\varepsilon_{\mathrm{r}}$ is the dielectric constant, a is the radius of the particle, $D$ is the separation distance, $\psi_{0}$ is surface potential, $\kappa$ is reciprocal of the Debye length. Debye length is given by ${ }^{34}$ 


$$
\kappa^{-1}=\sqrt{\frac{\varepsilon_{0} \varepsilon_{r} k T}{2 N_{A} e^{2} I}}
$$

where $I$ is the ionic strength of the electrolyte, $k$ is the Boltzmann constant, $T$ is the absolute temperature, $N_{A}$ is the Avogadro number, and $e$ is the elementary charge. Debye length is the measure of a charge carrier's net electrostatic effect in solution, and how far those electrostatic effects persist. A Debye sphere is a volume whose radius is the Debye length, in which there is a sphere of influence, and outside of which charges are electrically screened. The notion of Debye length plays an important role in colloids.

The thickness of the double layer depends on the ionic strength of the dispersion medium. For 1:1 electrolytes, the ionic strength is proportional to the concentration $c$. At low ionic strengths (electrolyte $c=10^{-3}$ ), the thickness of the double layer is about 5$10 \mathrm{~nm}$, which is of the same order as the van der Waals attraction. This explains the observation of charge stabilization in dispersion media of low ionic strength. The thickness of the double layer is reduced significantly with increasing the ionic strength. At ionic strengths for electrolyte $c>10^{-1} \mathrm{M}$, the thickness of the double layer is less than 1 $\mathrm{nm}$. In that case, the range of double layer electrostatic repulsion is usually insufficient to counterbalance the van der Waals attraction. This accounts for the fact that most chargestabilized dispersions coagulate when increasing the ionic strength of the dispersion medium. Hence, one great disadvantage of charge stabilization of particles is its great sensitivity to the ionic strength of the dispersion medium. In addition it only works in polar liquids which can dissolve electrolytes. However, due to the advantages in simplicity and cost, charge stabilization is still widely used in stabilizing dispersions in aqueous media. 


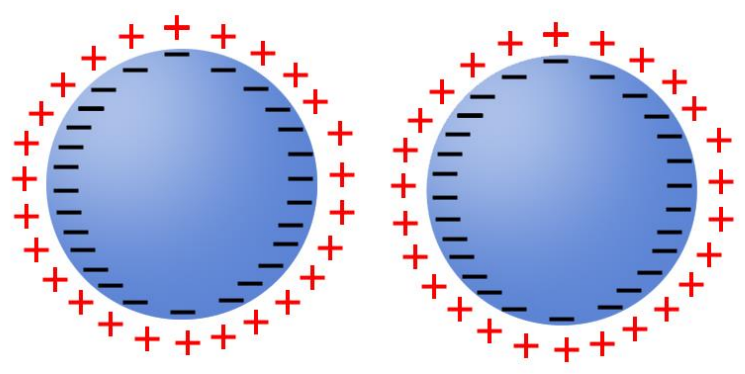

Figure 1.3 Schematic illustration of electrostatic stabilization.

\subsubsection{Steric Stabilization}

Steric stabilization provides an alternate route of controlling colloidal stability that can be used in aqueous and non-aqueous systems. In this approach, absorbed polymeric molecules are used to create steric repulsion. When two particles with adsorbed polymer layers approach each other at a distance of less than twice the thickness of the adsorbed layer, an interaction between the two layers takes place (Figure 1.4). The degree of stabilization can be defined quantitatively in terms of the energy change occurring upon the interaction of the adsorbed layers. The Gibbs free energy change $\Delta G$ of the overlap interaction of the adsorbed layers is expressed as $\Delta G=\Delta H-T \Delta S$. If $\Delta G$ is negative upon the overlap of the adsorbed layers, flocculation or coagulation will result, and if $\Delta G$ is positive, stabilization will result. Under isothermal conditions, the stability is then a function of the enthalpy change, $\Delta H$ and the entropy change, $\Delta S$. In one entropic stabilization theory, it is assumed that a second surface approaching the adsorbed layer is impenetrable. Thus, the adsorbed layer is compressed and the polymer segments present in the interaction region lose configurational entropy. That is, the polymer segments occupy fewer possible configurations in the compressed state than in the uncompressed 
state. This reduction in entropy increases $\Delta G$, producing the net effect of repulsion between the particles and thus preventing the particles from flocculating. ${ }^{51}$

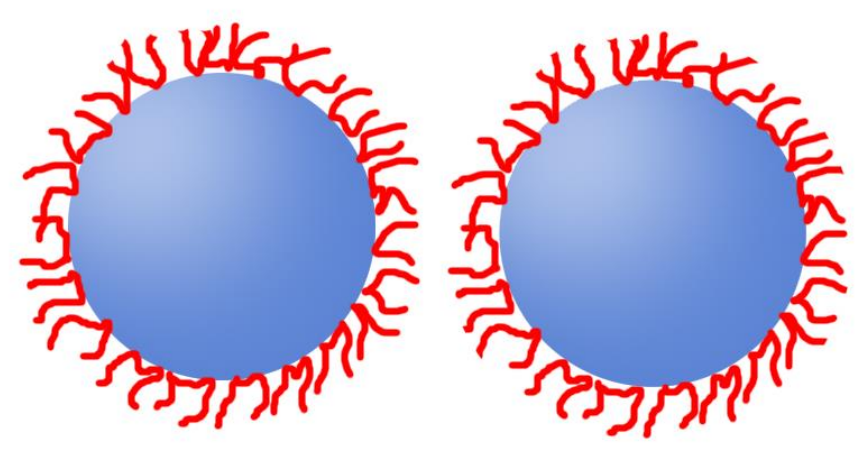

Figure 1.4 Schematic illustration of steric stabilization.

Steric stabilization has several distinct advantages over electrostatic stabilization:

(1) Relative insensitivity to the presence of electrolytes. For instance, for 1:1 electrolytes, a charge-stabilized dispersion will not be stable and coagulate when the concentration of electrolytes exceeds the $10-{ }^{1} \mathrm{M}$ limit. The dimensions of polymer chains display no such dramatic sensitivity and sterically stabilized dispersions are relatively insensitive to the presence of electrolyte.

(2) Equal efficacy in both aqueous and nonaqueous dispersion media. Charge stabilization is less effective in nonaqueous dispersion media than it is in aqueous media. This is primarily due to the low relative dielectric constant $(<10)$ of most nonaqueous media. In contrast, steric stabilization is effective in both nonaqueous media and aqueous media. This explains why steric stabilization is usually preferred for nonaqueous dispersion media.

(3) Equal efficacy at both high and low solids content. In charge stabilization in nonaqueous media, the thickness of the double layers can be so large, (due to the low 
dielectric constant of the dispersion medium), that the mere preparation of high solids dispersions forces the particles too close together which then leads to coagulation. In aqueous dispersion media, the preparation of charge-stabilized particles at high solids dispersions is often difficult because of the gel formation induced by the interactions between the double layers surrounding each particle.

(4) Reversibility of flocculation. The coagulation of charge-stabilized particles (induced by the addition of electrolyte) is usually irreversible by subsequent dilution. In contrast, flocculation of sterically stabilized dispersions (induced by the addition of a nonsolvent for the stabilizing moieties) can usually be reversed spontaneously by mere dilution of the nonsolvent concentration to a suitably low value. This difference is due to the fact that sterically stabilized dispersions may be thermodynamically stable while charge stabilized dispersions are only thermodynamically metastable. As a consequence, for charge stabilized dispersions, the coagulated state represents a lower energy state and the coagulation can be reversed only after input of work into the system. Another important consequence of the thermodynamic stability of sterically stabilized dispersions is that they can re-disperse spontaneously after drying.

\subsubsection{Electrosteric Stabilization}

Polyelectrolyte species are widely used additives that can impart electrostatic and steric stabilization to a given colloidal dispersion. ${ }^{54}$ Such systems are often referred to as electrosterically stabilized (Figure 1.5). Polyelectrolytes contain at least one type of ionizable group (e.g., carboxylic or sulfonic acid groups), with molecular architectures that range from homopolymers, such as poly (acrylic acid), to block copolymers with one or more ionizable segments. Polyelectrolyte adsorption is strongly influenced by the 
chemical and physical properties of the solid surfaces and solvent medium. ${ }^{55}$ For example, adsorption is strongly favored when polyelectrolyte species and the colloid surfaces of interest carry opposite charges ${ }^{56}$ At small adsorbed amounts, such species can promote flocculation either via surface charge neutralization or bridging mechanisms. At higher adsorbed amounts, particle stability increases because of long-range repulsive forces resulting from electrosteric interactions. ${ }^{57}$ For a given system, the adsorption behavior and conformation of polyelectrolyte species can be modulated by tailoring solvent conditions (e.g., $\mathrm{pH}$ and ionic strength). ${ }^{58,59}$
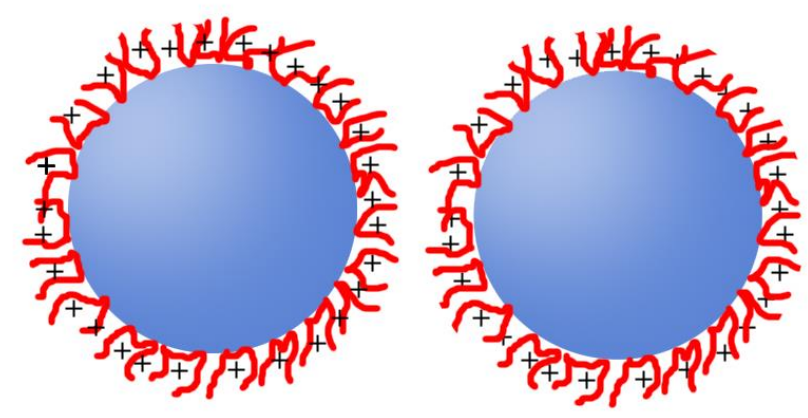

Figure 1.5 Schematic illustration of electrostatic stabilization.

\subsubsection{Nanoparticle Haloing - A New Colloidal Stabilization Mechanism}

As summarized above, traditional methods to stabilize a suspension typically involve tuning of the effective interactions through charged groups or through grafting short polymer chains onto the colloidal surface. These mechanisms, however, pose serious problems in certain situations, such as the fabrication of close-packed colloidal crystals, where they cause an increase in the lattice spacing of the sedimented colloids and thus lead to cracking of the crystal upon drying. The attached surfactants on colloidal surfaces may also change the thermal properties of the colloidal particles and solvent. 
Since 2001, a novel method called "Nanoparticle Haloing" proposed by Tohver et al. has provided a new perspective on the stabilization of colloidal suspensions. ${ }^{98-100}$ They observed that negligibly charged silica suspensions can be stabilized by adding small amounts of highly charged $\mathrm{ZrO}_{2}$ nanoparticles due to the formation of a non-adsorbing layer of highly charged nanoparticles around weakly charged colloidal microspheres (Figure 1.6). Subsequently, this new stabilization method through nanoparticles has been successfully applied to several other colloidal-nanoparticle systems, such as silicapolystyrene and silica-alumina. ${ }^{101-103}$ Further studies have shown that the nanoparticle halo is not confined to mixtures of weakly charged colloids and highly charged nanoparticles, but can also be observed in mutually charged microparticle/nanoparticle dispersions. ${ }^{104}$ By means of ultrasmall-angle X-ray scattering, Zhang et al. quantified that, at zirconia volume fraction of $10^{-3}$, the self-organized nanoparticle layer is approximately $2 \mathrm{~nm}$ away from the colloidal surface at $\mathrm{pH} 1.5$ which is nearly equal to the Debye length. ${ }^{105}$

In order to understand the mechanism of the nanoparticle-regulated stabilization, recent works have been focused on investigation of the interactions between colloidal surfaces in the presence of nanoparticles through both simulation and experimental methods. Different Monte Carlo simulation approaches and a modified Poisson-Boltzmann equation have been introduced to investigate critical conditions for the formation of nanoparticle halos. ${ }^{106-111}$ By using Atomic Force Microscopy (AFM), ${ }^{112}$ we are the first group that directly measured the interaction force between colloidal surfaces in charged nanoparticle suspensions, providing insight into understanding the fundamental mechanism of nanoparticle haloing. Direct force measurements will be 
detailed in the next sections $(1.4 \& 1.5)$. The experimental interaction force curves demonstrate that an electrostatic repulsion between weakly charged colloidal surfaces is induced by the addition of highly charged nanoparticles. Once the effective repulsion is sufficient to overcome van der Waals attraction above a critical nanoparticle concentration, the colloidal microsphere-plate system would be prevented from aggregation. Moreover, a force barrier at $\sim 2 \mathrm{~nm}$ in the force curve has been measured at volume fraction of $10^{-5}$ which suggests the distance between nanoparticle halo and colloidal surfaces is approximately the system's Debye length. This dissertation is a continuation of that previous study. The effect of nanoparticle properties such as size and concentration on the formation of the nanoparticle halo has been studied. A theoretical model of estimating interactions in the nanoparticle haloing system has been developed as well.

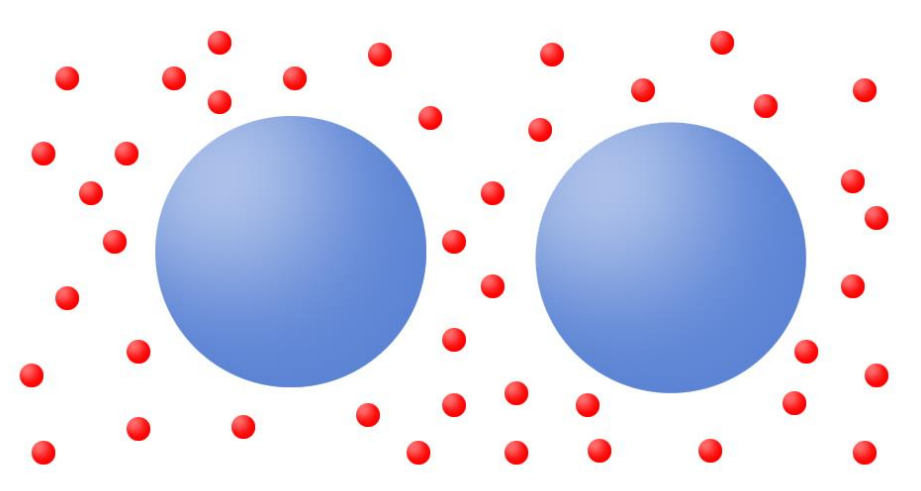

Figure 1.6 Schematic illustration of nanoparticle-halo stabilization.

The stabilization mechanism of nanoparticle haloing may be of particularly high value in applications where steric stabilization using adsorbed species may unfavorably alter the particle size or may interfere with reactivity or availability of the surface, such as in ceramics processing, chemical-mechanical planarization, colloidal surface functionalization, or catalysis. ${ }^{113}$ 


\subsection{Interaction Measurements among Colloidal Particles}

As mentioned above, there have been well-developed theories that describe the inter-particle interactions in colloidal suspensions, most of which can be resolved either analytically or numerically in terms of the underlying fundamentals. Moreover, the past several decades have seen the advent of accurate direct measurements of the forces acting between particles as a function of surface separation in liquids. These have facilitated the validation of the inter-particle interaction theories and the further insight into more complex phenomena.

The first direct measurement of inter-particle forces was conducted by Derjaguin et al. who measured the attractive van der Waals forces between a convex lens and a flat glass surface in vacuum. ${ }^{114,115}$ An electro-balance was used to measure the forces and an optical technique to detect the distance between two glass surfaces. The distance is in the range of $100-1000 \mathrm{~nm}$, and the results fell within $50 \%$ of theoretical predicted van der Waals forces. Derjaguin et al.'s work paved the way for the highly accurate techniques that are employed nowadays for measuring the interactions between surfaces in vapors and liquids. Ever since the first direct measurements of forces between surfaces, various techniques have been developed, which allow for the full force laws to be measured at the angstrom level. The first accurate, direct measurements of forces between macroscopic solid surfaces immersed in aqueous electrolytes were reported in 1978 by Israelachvili and Adams ${ }^{116}$ using an instrument referred to as a surface forces apparatus (SFA), which is based on the use of muscovite mica, a material originally suggested by Debye. The separation distance between these molecularly smooth crystals could be accurately measured using interferometry and the force obtained by measurement of the deflection 
of a spring. Although problems were encountered, the forces in some cases ${ }^{117}$ were found to be in complete agreement with the DLVO theory.

Although the SFA technique has been successfully applied to the detailed study of surface interactions, it is limited by the requirements that the substrates are: (1) composed of thin (micrometre) sheets, (2) molecularly smooth on both faces over a relatively large area of several square centimeters, and (3) semitransparent. ${ }^{118,119}$ So far, mica, due to its molecularly smooth surface and ease of handling, has been the primary surface material used in SFA studies.

Another important, although less direct, technique for measuring forces between macromolecules or lipid bilayers is the osmotic stress method. ${ }^{120,122}$ In the osmotic stress method a dispersion of vesicles or macromolecules is equilibrated with a reservoir solution containing water and other small solutes, which can freely exchanged with the dispersion phase. The reservoir also contains a polymer which cannot diffuse into the dispersion. The polymer concentration determines the osmotic stress acting on the dispersion. The spacing between the macromolecules or vesicles is measured by X-ray diffraction. In this way one obtains pressure-versus-distance curves.

During the last $10-15$ years a new technique called total internal reflection microscopy (TIRM) was developed. ${ }^{123}$ Using TIRM, the distance between a single microsphere immersed in a liquid and a transparent plate can be monitored with typically $1 \mathrm{~nm}$ resolution. The distance is calculated from the intensity of light scattered by the sphere when illuminated by an evanescent wave through the plate. From the equilibrium distribution of distances sampled by Brownian motions the potential energy-versus- 
distance can be determined. TIRM complements force measurements with the SFA because it covers a lower force range.

These techniques have allowed accurate measurement of surface and inter-particle forces and led to improved understanding in this field. However, only a limited number of systems could be investigated because of restrictions to the material properties and the complexity of the equipment. In contrast, the Atomic Force Microscopy (AFM) is relatively easy to use.

\subsection{Atomic Force Microscopy}

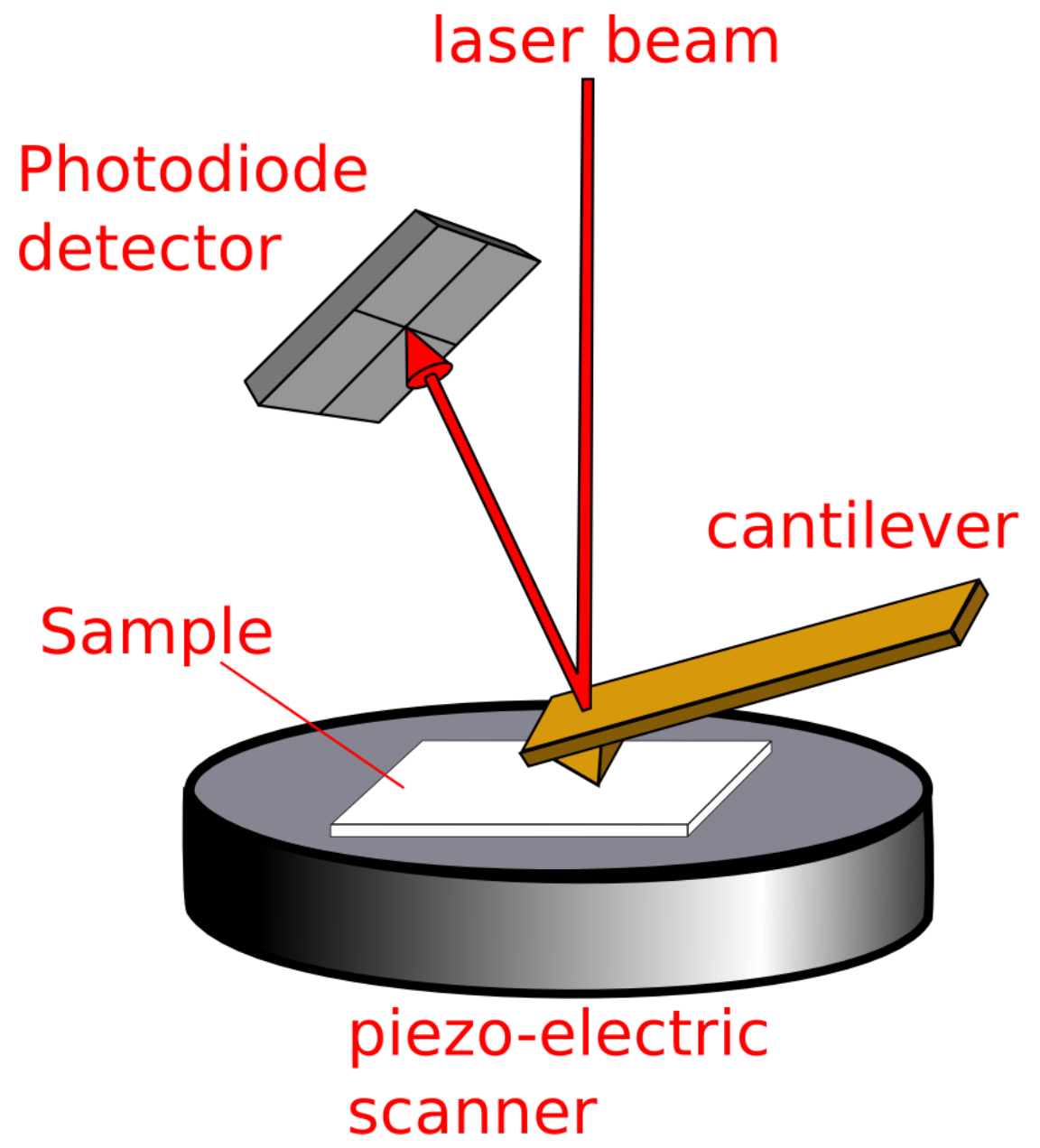

Figure 1.7 Schematic of an atomic force microscope. 
The Atomic Force Microscopy (AFM) was developed following the dramatic appearance of the scanning tunneling microscope, and both owe their development to the availability of improved piezoelectric devices, digital signal processing, and extended PC storage. ${ }^{124}$ The AFM uses a light lever to detect the deflection of a fine cantilever spring as it interacts with the substrate surface beneath it using a piezoelectric transducer. A laser light is focused onto the back of the cantilever spring. The reflected light is directed onto a split photodiode detector, which produces a current signal proportional to the cantilever deflection. The approach speed and relative particle-surface position are accurately controlled by application of a voltage across the piezoelectric ceramics. In the force measurements, motion in the $x$ and $y$ directions is disabled and the piezoelectric tube is used to move the probe in the $z$ direction and the cantilever deflection is continuously measured. The deflection of the cantilever can be converted to a force using Hooke's law and the known spring constant of the cantilever.

The AFM device has also the advantage of being able to image non-conducting surfaces to high resolution in air or even in liquid, which enables the study of a wide range of solid - liquid interfaces under real conditions. A topographic image of the surface is obtained by monitoring the vertical movement of a piezoelectric crystal required to maintain a constant spring deflection, as the tip of the spring is scanned across the surface also by the piezo. ${ }^{125}$ This information is stored on the computer with the relative position and then used to generate a three-dimensional image of the surface.

In 1991, a commercial AFM device was adapted to detect the spring deflection resulting from the interaction of a fine colloidal particle attached to the top of a cantilever with a flat substrate of the same material, immersed in a range of aqueous electrolyte 
solutions. ${ }^{126}$ Using this technique, colloidal forces were measured directly for the first time. The results obtained using a silica glass colloid and flat substrate were found to be in good agreement with the DLVO theory down to surface separations of about 3-4 nm. The change in decay lengths with added electrolyte also agreed with theory. ${ }^{127}$ In addition, the surface electrical potentials extracted from the DLVO theoretical fits are consistent with values obtained using other techniques, such as microelectrophoresis. ${ }^{128}$

\subsubsection{Working mode}

AFM has three primary modes of operation: contact mode, non-contact mode, and tapping mode or intermittent.

(1) Contact Mode. Contact mode is the most direct AFM imaging mode. Here, the force on the cantilever is kept constant and the distance between the tip and the sample surface is sufficiently small to allow a core repulsion effect. As the probe is moved over the sample surface, the topography changes cause variations in the tip-sample interaction. The force incident on the cantilever tip is altered and the equilibrium between the elastic force of the deflected cantilever and applied force changes. The deflection is detected by photodiode deflection of a light beam, which is fed back to the piezo controller, which adjusts the $Z$-position of the sample to restore the set point force and deflection.

Constant height mode involves the cantilever in a fixed position with respect to the piezo. The constant force mode however, involves a total force between the cantilever tip and sample is held constant by means of a feedback loop. The scanner moves up and

down to keep the cantilever deflection constant as the topography changes. ${ }^{129}$ This method is capable of generating accurate topographies but is strongly influenced by tip geometry and would potentially damage the sample surface or tip. 
(2) Non-Contact Mode. In this mode the AFM cantilever is vibrated near the sample surface. The distance between the tip and the surface maintains that the attractive van der Waals force is dominant. The cantilever is held at a $5-10 \mathrm{~nm}$ distance away from the surface and oscillated at a resonant frequency.

The force between the tip and sample is several orders of magnitude lower than in the contact mode. Soft surfaces can be analyzed and samples are not damaged or contaminated through contact. It has been shown that this mode is more effective for imaging biology membranes. ${ }^{130-132}$ However, this technique is only applicable for use in air, where the van der Waals forces dominate. In liquids there are frequently additional repulsive forces, which mitigate the Van der Waals interaction.

(3) Intermittent-Contact/Tapping Mode. This is a hybrid of the preceding methods where the cantilever being vibrated is held at a distance closer to that of contact imaging. A resonant frequency is reached and then contact is made with the sample. With this mode, an image is obtained by monitoring the changes in the cantilever oscillation amplitude as the tip to sample distance changes with sample topography. It combines qualities of both the contact and noncontact modes by collecting sample data and oscillating the cantilever tip at or near its natural resonance frequency while allowing the cantilever tip to impact the target sample for a minimal amount of time. The advantage here is that the lateral forces are reduced and the energy loss from tip-sample contact naturally leads to an amplitude loss, which is measured to identify surface features. ${ }^{133}$

\subsubsection{Force Measurements}

Image contrast arises because the force between the tip and sample is a function of both tip-sample separation and the material properties of the tip and sample. To date, 
in most applications image contrast is obtained from the very short-range repulsion, which occurs when the electron orbitals of tip and sample overlap (Born repulsion). However, further interactions between tip and sample can be used to investigate properties of the sample, the tip, or the medium in between. These measurements are usually known as "force measurements". In an AFM force measurement the tip attached to a cantilever spring is moved towards the sample in normal direction. Vertical position of the tip and deflection of the cantilever are recorded and converted to force-versusdistance curves, briefly called "force curves".

In a force measurement, the AFM cantilever is moved up and down in the vertical direction by applying a voltage to the piezoelectric translator, while measuring the cantilever deflection. After attaching one microsphere to the cantilever tip and a second microsphere to the surface of the support, it is possible to measure to the force of interaction between colloidal particles. According to Hooke's law $(F=k d$, where $F$ corresponds to the driving force, $k$ is the spring constant of the cantilever, $d$ is the cantilever deflection), the force is directly proportional to the cantilever deflection. 


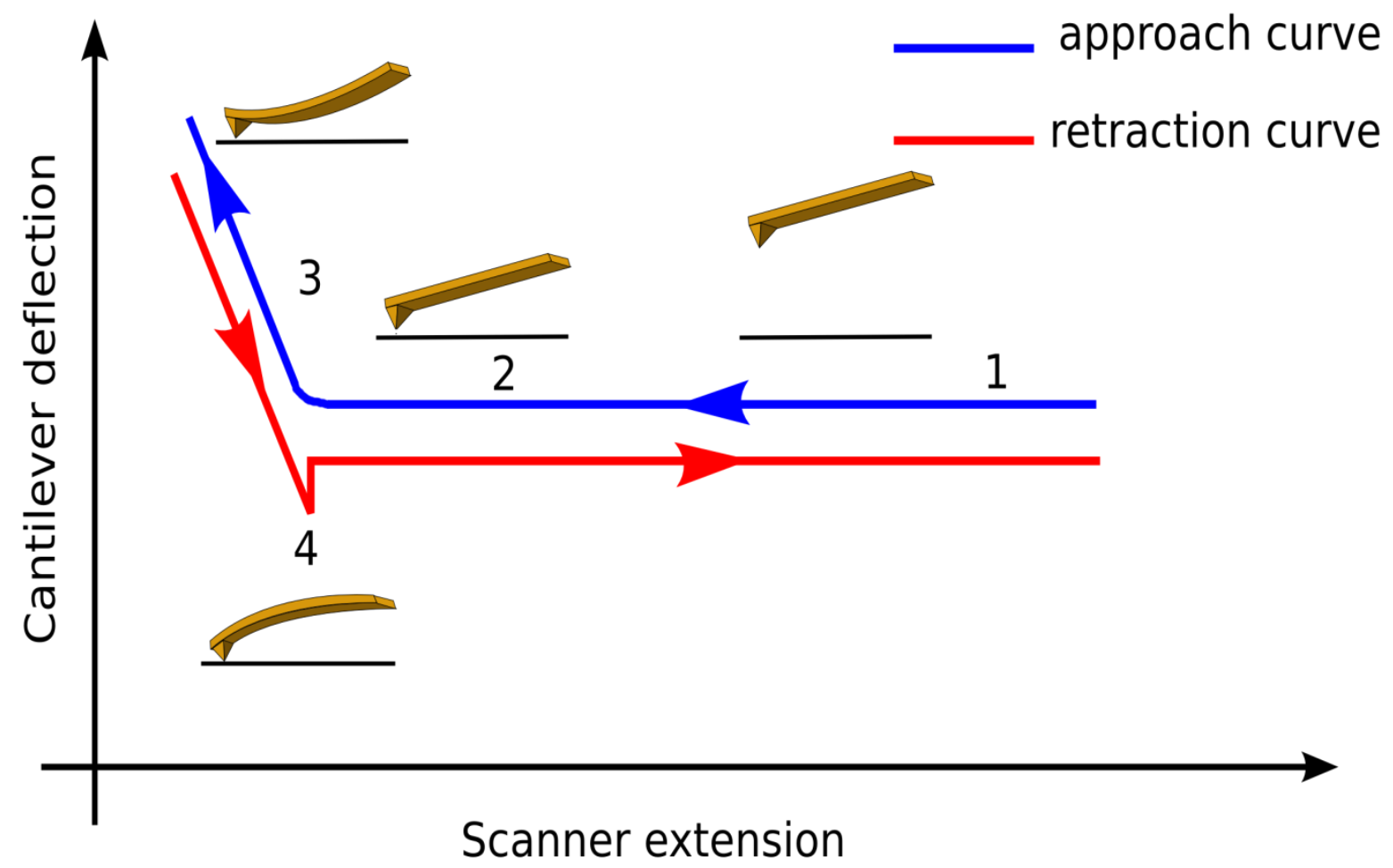

Figure 1.8 Schematic of a typical cantilever deflection-vs.-z scanner height curve.

In force measurement mode the AFM tip approaches and is pushed into the sample until a predefined force is reached; at this point the tip is retracted again. During this complete cycle the position of the tip as well as the force exerted on the cantilever are accurately monitored, resulting in a force curve (Figure 1.8). At the beginning (Fig.1.8_1), the distance between the probe and the surface is large and probe-surface interactions are absent. As the probe approaches the surface, if the tip experiences repulsion, the cantilever deflects upward (Fig.1.8_2); if the tip detects attraction, the cantilever deflects downward (Fig.1.8_4). As the tip is brought closer to the surface, it comes in a contact with the surface when attractive forces are greater than the stiffness of the cantilever and the repulsive forces (Fig.1.8_3). Once the tip is in contact with the surface, a nearly linear (constant) compliance region appears. After the cantilever force 
reaches the desired force set-point value, the process is then reversed while the cantilever is retracted.

\subsubsection{Colloidal Probe AFM Technique}

The colloidal probe technique was introduced in 1991 . Ducker et al. ${ }^{126}$ glued silica spheres onto cantilevers while Butt used glass spheres ${ }^{124}$. The colloid probes are usually prepared by attaching a sphere to a standard AFM cantilever with resin or glue. During the attachment procedure, the cantilever is mounted into the head of the AFM, as seen in Figure 1.9. The sphere and a thin layer of the glue are placed next to each other on a glass surface. A micromanipulator is used to coat the end of the cantilever with glue and then pick up a single sphere. Great care should be taken not to coat the lower surface of the sphere with the glue.

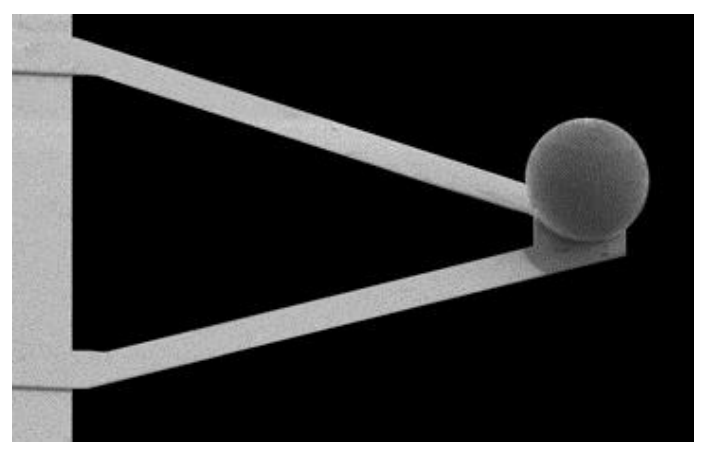

Figure 1.9 SEM images of a colloid probe: a silica sphere $(1 \mu \mathrm{m})$ attached to the apex of AFM cantilever.

With the development of colloidal probe technique, it is possible to measure directly the DLVO forces between several materials which are of special interest in colloidal science, e.g. silica, and silicon nitride; copper and nickel; zinc and lead sulphide; titanium oxide; zirconia; and alumina. In addition the spheres held on the end of 
the cantilever can be coated with different polymers in order to investigate the interaction of the polymer with relevant surface. ${ }^{135-137}$

We are the first group that has directly measured the interaction forces between a silica microsphere and plate in highly charged nanoparticle solutions, which provides a new perspective on understanding the fundamental mechanism of nanoparticle haloing system. In this work, AFM force measurement is continually employed to investigate the interaction between colloidal surfaces in zirconia nanoparticle suspensions with varying concentrations and sizes, in order to elucidate the fundamental mechanism of nanoparticle haloing. 


\section{CHAPTER 2}

\section{TECHNIQUES}

\subsection{Force Measurements by Atomic Force Microscopy}

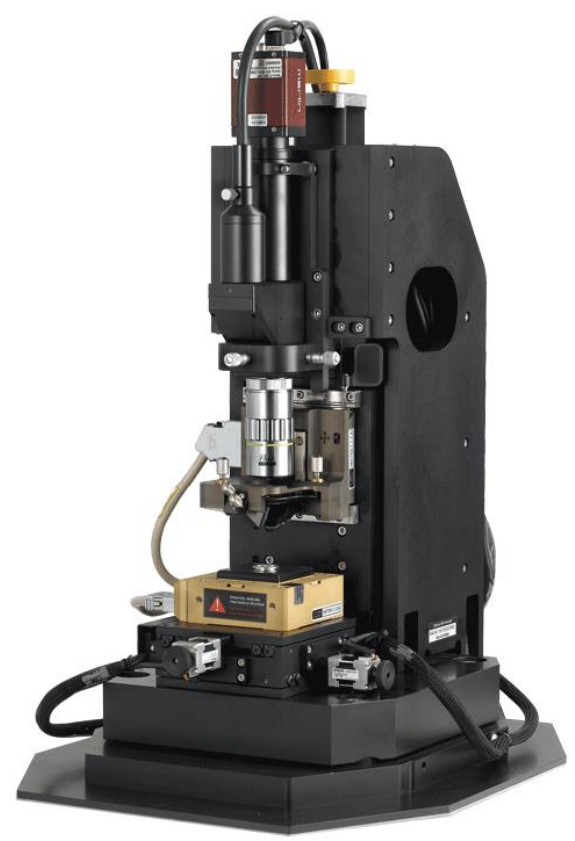

Figure 2.1 A representation of the XE100 AFM used during experiments. ${ }^{141}$

As detailed in the introduction, CP-AFM provides a relatively easy and accurate method for direct force measurement between colloidal microspheres. Therefore, in this work an XE100 AFM (Figure 2.1) was used to perform the scanning and the force measurement experiments in order to investigate the fundamental mechanism of colloidal stabilization through nanoparticles. This study is of particular interest in measuring interactions between colloidal particles in the medium of a nanoparticle 
suspension. By pre-attaching a colloidal particle onto an AFM cantilever and another particle of the same material on the sample stage, it's possible to measure the interaction between colloidal particles while the tip is approaching the surface. In practice, however, it is of great difficulty to align two microspheres $(\sim 1 \mu \mathrm{m})$ coaxially. Thus the experimental interaction measurements were simplified from a microsphere-microsphere system (Fig 2.2a) to microsphere-plate (Fig 2.2b) by using a colloidal probe to approach a plate substrate of the same material, which can be taken as interaction between colloidal particles with dramatically different diameters.

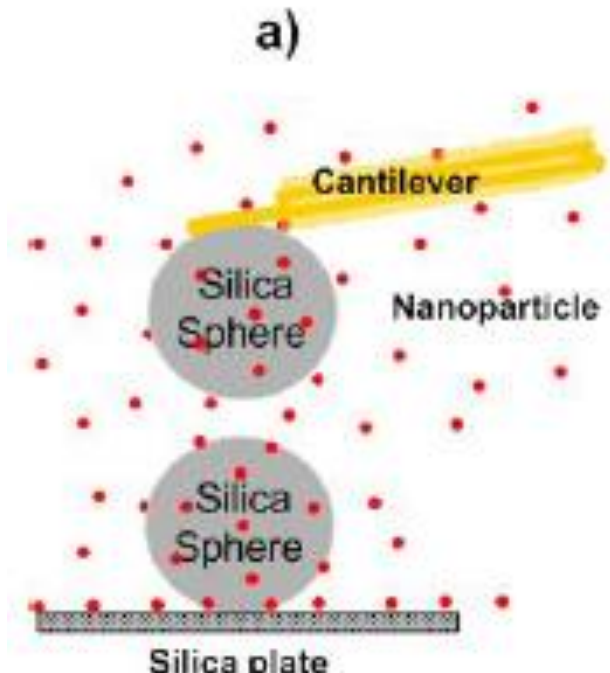

Silica plate

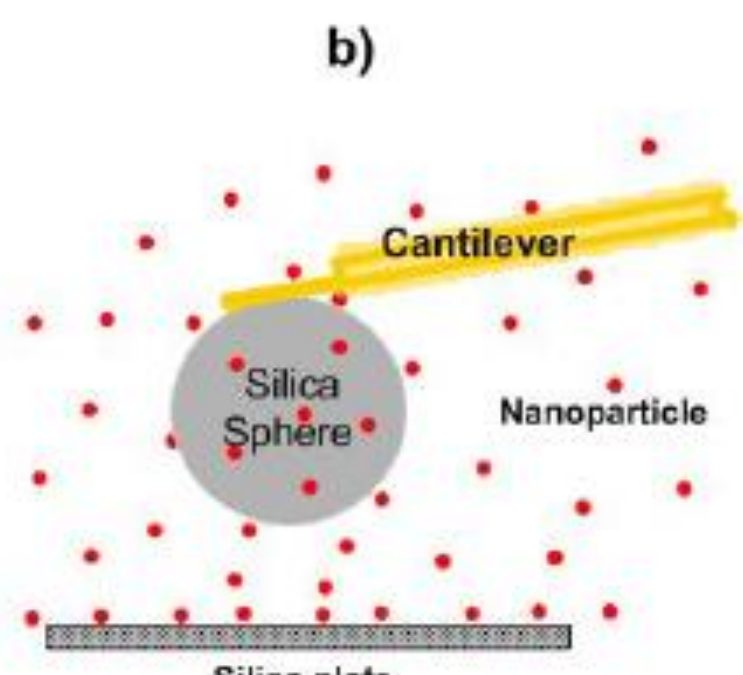

Silica plate

Figure 2.2 Schematic description of AFM force measurement for a) sphere-sphere system, b) sphere-plate system.

The direct result of a force measurement is a measure of deflection of the cantilever as a function of the scanner displacement. These data can directly be converted to force vs. distance profiles by defining the zero points for both the force and the separation distance (Figure 2.3) ${ }^{142}$ The zero separation distance was chosen where 
cantilever deflection was linear with sample displacement at high force, and the zero force occurs when deflection maintained a constant value at large separation distance. ${ }^{143-}$ 148

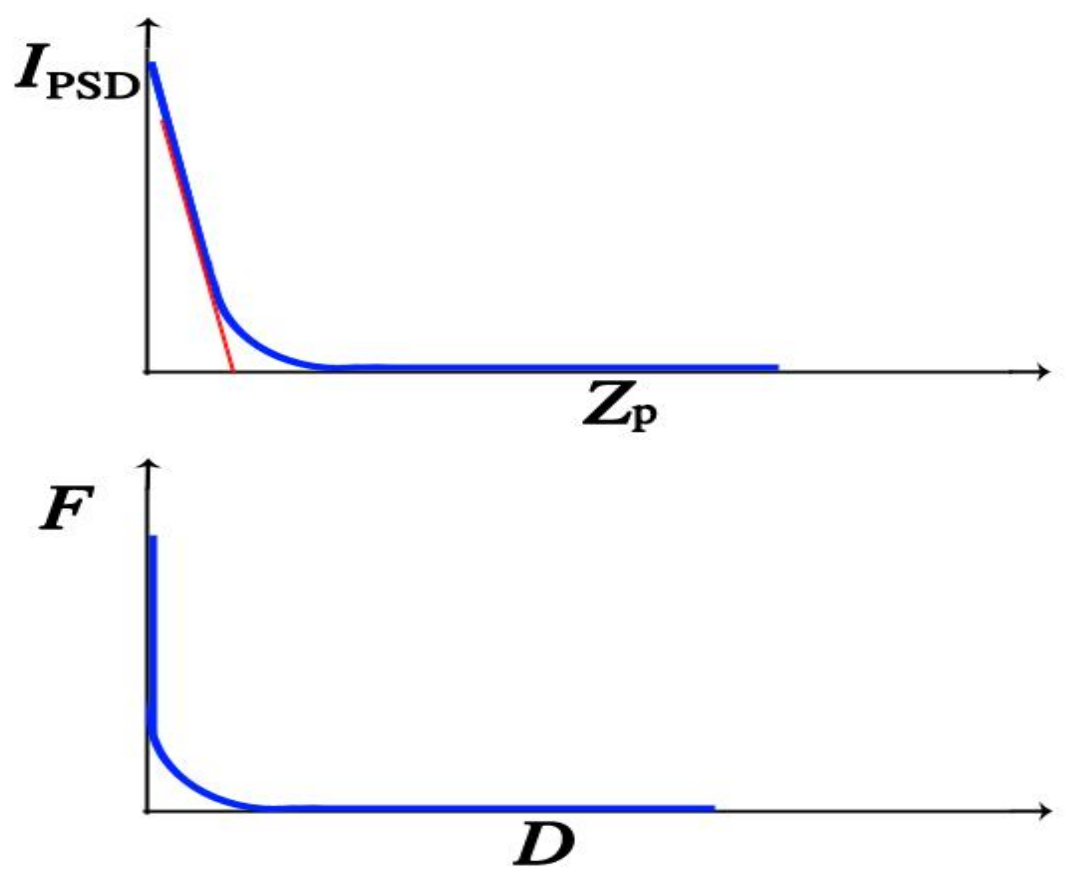

Figure 2.3 Schematic of typical Piezo Scanner Deflection-vs.-Piezo Height curve and corresponding force-vs.-distance curve.

\subsection{Zeta Potential}

Zeta potential is a scientific term for electro-kinetic potential in colloidal systems. When charged particles are present in a medium, an electric double layer will be developed. The double layer consists of ions, which are firmly bound to the surface (Stern layer) and ions, which are loosely bound (diffuse layer) to the surface (see figure 2.4). From a theoretical viewpoint, the zeta potential is the electric potential in the interfacial double layer (DL) at the location of the slipping plane versus a point in the 
bulk fluid away from the interface. In other words, zeta potential is the potential difference between the dispersion medium and the stationary layer of fluid attached to the dispersed particle. A value of $25 \mathrm{mV}$ (positive or negative) can be taken as the arbitrary value that separates low-charged surfaces from highly charged surfaces. The significance of zeta potential is that its value can be related to the stability of colloidal dispersions. The zeta potential indicates the degree of repulsion between adjacent, similarly charged particles in dispersion. For molecules and particles that are small enough, a high zeta potential will provide stability, i.e., the solution or dispersion will resist aggregation. When the potential is low, attraction exceeds repulsion and the dispersion will break and flocculate. So, colloids with high zeta potential (negative or positive) are electrically stabilized while colloids with low zeta potentials tend to coagulate or flocculate. ${ }^{149-150}$

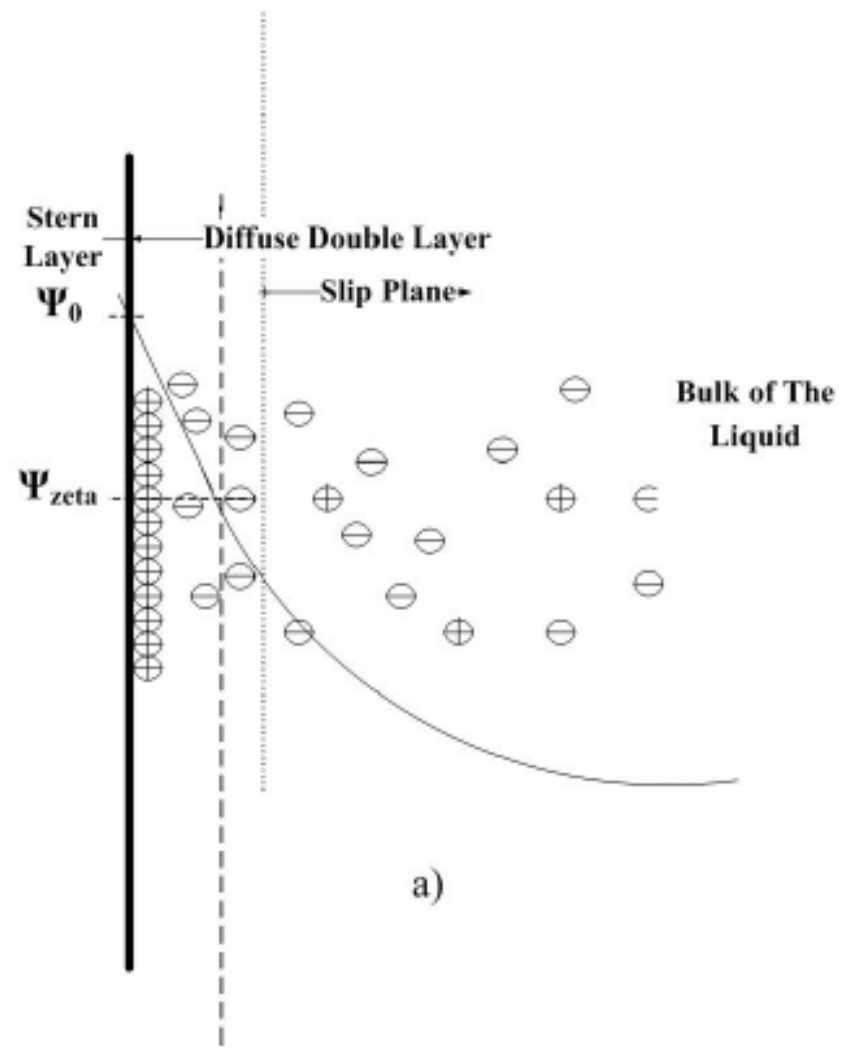

Figure 2.4 The double layer of a plane. 
In summary, zeta potential is a measure of the magnitude of the electrostatic repulsion between colloidal particles, and is a fundamental parameter known to affect stability. Its measurement provides the causes of dispersion, aggregation or flocculation, which can be applied to improve the formulation of dispersions, emulsions and suspensions. In this work, a 90 Plus-Zeta particle size analyzer (Brookhaven Instruments, Holtsville, NY) was used to measure zeta potentials of binary mixtures of colloidal microspheres and nanoparticles of different compositions. These measurements bring detailed insight into the effect of additional nanoparticles on the stability of colloidal suspensions.

The particle analyzer was used to measure sizes of colloidal particles as well. To determine the size of the particles, it is necessary to measure the Brownian motion of the particles in the sample using Dynamic Light Scattering (DLS), also known as Photocorrelation Spectroscopy (PCS). Small particles will move quickly and larger particles will move slower. The particles are illuminated with a laser and the intensity fluctuations of the scattered light are analyzed. If a small particle is hit by a light source the particle will scatter the light in all directions. If many particles are present in the system a speckle pattern will be formed which consists of bright and dark areas. The bright areas are regions where the light scattered by the particles has the same phase and interferes constructively to form a bright patch. The dark areas are regions where the phase additions are mutually destructive and cancel each other out. The Stokes-Einstein equation relates the size of the particle with its speed due to Brownian motion. Since the particles move, the intensity appears to fluctuate. The instrument measures the rate of the intensity fluctuation and from there calculates the size of the particles. 


\subsection{Nanoparticle Adsorption}

In order to investigate the degree of nanoparticle deposition on colloidal surfaces at different nanoparticle concentrations, a scanning electron microscope (SEM) (FEI Nova 600) was used to obtain images of the nanoparticles adsorbed on silica glass. The accelerating voltage was $20 \mathrm{kV}$ and the beam current was $5.15 \mathrm{pA}$. The image mode was a secondary electron image. An SEM is a type of electron microscope that produces images of a sample by scanning it with a focused beam of electrons. The electrons interact with atoms in the sample, producing various signals that can be detected and that contain information about the sample's surface topography and composition. ${ }^{151}$ The electrons coming from an electron gun have a typical energy of 2-40 kV. The electron beam is demagnified into a probe of electrons. ${ }^{152}$ The probe of electrons with a diameter of $1-10 \mathrm{~nm}$ carrying a current of $10^{-9}-10^{-12} \mathrm{~A}$ is focused onto the surface and moved across the surface in parallel lines. ${ }^{151,153}$ The interaction of the electrons with the surface produces several phenomena, among them the emission of secondary electrons with an energy of 2-5 eV, and high energy backscattered electrons.

The limit between secondary electrons and backscattered electrons is drawn at 50 $\mathrm{eV}$. The secondary electrons are emitted from the sample and generated by inelastic collisions to high energy levels, so that the excited electrons can overcome the work

function before a deceleration to the Fermi level occurs. ${ }^{151}$ The backscattered electrons are electrons from the incident beam, which interact with atoms in the sample and are backscattered again. The intensity of both emissions, secondary and backscattered electrons, is sensitive to the angle at which the incident beam contacts the surface. The emissions are collected by the detectors and amplified. The resulting signal is used to 
control the brightness in a cathode ray tube (CRT) ${ }^{135}$ The CRT scan is synchronized with the beam scan, which allows the signals to be transferred point to point and a map of the scanned area can be displayed. The scanning electron microscopy image is a magnification of the topography of the sample, secondary or backscattered images can be obtained. The contrast of a backscattered SEM image depends on the intensity of the emitted backscattered electrons. When heavy atoms are present in the sample, more backscattered electrons will be produced and a brighter contrast is obtained. Therefore, local variations in average atomic number vary the contrast of the image. ${ }^{153}$ The interaction of the electrons with the sample produces other emissions: $\mathrm{X}$ ray photons, Auger electrons, and perhaps light. ${ }^{152}$ The spectrum of the x-radiation can be used for quantitative chemical microanalysis. Auger electrons are emitted from atomic layer close to the surface and give information about the surface chemistry. 


\section{CHAPTER 3}

\section{FORCE MEASUREMENTS BETWEEN COLLOIDAL SURFACES IN NANOPARTICLE SOLUSIONS BY USING CP-AFM}

\subsection{Introduction}

Tuning the dispersion behavior of colloidal microspheres is important in several industrially relevant products such as coatings, drug carriers and ceramics. ${ }^{1-10,}$ 154-161 Traditional colloidal suspension stabilizations involve controlling the effective interactions through charged groups or deposition of polymer chains onto the colloidal surface. ${ }^{162-164}$ However, these mechanisms have serious disadvantages, such as changing the thermal properties of the colloidal particles or contaminating the heat transfer media. $^{49}$

Since 2001, a novel method called "Nanoparticle Halo" proposed by Tohver et al.

has provided a new perspective on the stabilization of colloidal suspensions. ${ }^{98-100}$ They observed that negligibly charged silica suspensions can be stabilized by adding small amounts of highly charged $\mathrm{ZrO}_{2}$ nanoparticles due to the formation of a non-adsorbing layer of highly charged nanoparticles around weakly charged colloidal microsphere. Subsequently, this new stabilization method via nanoparticles has been successfully applied to several other colloidal-nanoparticle systems, such as silica-polystyrene and 
silica-alumina. ${ }^{101-103}$ Further studies have shown that the nanoaprticle halo is not confined to mixtures of weakly charged colloids and highly charged nanoparticles, but can also be observed in mutually charged microparticle/nanoparticle dispersions. ${ }^{104}$

With the new colloidal suspension stabilization regulated by nanoparticles successively developed experimentally, there is an obvious interest in the fundamental mechanisms responsible for this stabilization. In order to understand the mechanism of the nanoparticle-regulated stabilization, recent works have focused on the investigation of interactions between colloidal surfaces in the presence of nanoparticles through both simulation and experimental methods. Different Monte Carlo simulation approaches and a modified Poisson-Boltzmann equation have been introduced to investigate critical conditions for the formation of nanoparticle halos. ${ }^{106-111}$ Besides, Scheer and co-workers systematically investigated the effect of the variation of size ratio, volume fraction and charge on nanoparticle haloing by integral equation theory ${ }^{165}$. Quite recently, by means of ultrasmall-angle X-ray scattering, Zhang et al. quantified that, at zirconia volume fraction of $10^{-3}$, the self-organized nanoparticle layer is approximately $2 \mathrm{~nm}$ away from the colloidal surface at $\mathrm{pH} 1.5$ which is nearly equal to the Debye length. ${ }^{105}$ Unfortunately, there has yet been no direct quantitative measurement of the colloidal interparticle forces within this stabilization system.

For colloidal particles, the total interaction force is the summation of a number of surface forces such as the attractive van der Waals force, the repulsive electrostatic force, structural forces ${ }^{166}$, depletion forces, ${ }^{167-169}$ hydration forces ${ }^{170,}$ 171, and hydrophobic forces $^{172}$. There are three techniques that are primarily employed to measure the 
interaction force between microscopic surfaces: Surface Force Apparatus (SFA), Total Internal Reflectance Microscopy (TIRM), and Atomic Force Microscopy (AFM).

Surface Force Apparatus (SFA) was first used to directly measurement the surface forces between macroscopic surfaces in $1978 .{ }^{116}$ The SFA is a versatile instrument that can be used in liquid, ambient, and vacuum environments. SFA has the advantage of obtaining direct measurements of separation and refractive index of the intervening medium simultaneously. Limitations include having to use macroscopic $(\mathrm{cm})$, transparent smooth surfaces which limits direct application of the results to a few macroscopic materials, and makes the direct force measurement for colloid particles impossible. Finally, it is extremely difficult to conduct a contaminant free experiment because there is such a large surface area under study.

$\mathrm{TIRM}^{123}$ has a primary advantage that the particle is not attached to a spring which leads to very sensitive detection of the force between a freely rotating particle and surface $(0.01 \mathrm{pN})$. However, it is also the primary drawback. In order to generate the potential energy profile, a stable position between the particle and surface is required for TRIM, which means the force must be measured in solution. If an attractive force between the surfaces is sufficiently strong, the force cannot be measured due to the fact that the particle simply falls into the surface which limits applications of TIRM to cases where a sufficient van der Waals or hydrophobic attraction exists between the surface and particle. Moreover, interaction forces cannot be measured in vacuum or air by TRIM.

The development of colloid probe AFM (CP-AFM) where a particle of interest was attached to the end of the cantilever has made it possible to directly measure and quantify the total interaction force as a function of separation distance between a 
colloidal particle and a microscopic flat surface in an electrolyte solution. ${ }^{126}$ It has been successfully applied to measure interactions between two similarly charged surfaces ${ }^{173}$ as well as between dissimilarly charged surfaces ${ }^{174,}{ }^{175}$. The CP-AFM has been further extended to measure the surface interaction between two colloidal particles with diameters up to $2 \mu \mathrm{m} .{ }^{176}$ The majority of the CP-AFM force measurements involved the measurement of double layer repulsion between two charged surfaces in an electrolyte solution. Explanation of the interaction force between two surfaces in a colloidal suspension is of even more interest due to its importance in industrial and medical applications. Recently, the surface force measurement for a zirconia sphere/flat system in a dispersant suspension has been utilized to explain the steric stabilization of nanozirconia dispersions. ${ }^{177}$ The interaction forces between two hard surfaces in sodium dodecyl sulfate containing aqueous systems have also been studied by CP-AFM. ${ }^{178}$ Subsequently, Drelich and co-workers have measured the colloidal surface forces between different surfaces in alumina/silica nanoparticle suspensions. ${ }^{179,}{ }^{180}$ Because the CP-AFM technique provides an easy and accurate method to qualify the interaction force between colloidal surfaces in both electrolyte and nanoparticle solutions, it is used to investigate the nanoparticle-haloing system in our work.

In this chapter, a comprehensive experimental study has been made to investigate the interaction force between a neutral silica microsphere and plate in presence of highly charged nanoparticles as a function of the nanoparticle concentration. Despite direct measurement of the interaction force between two silica spherical particles is obviously more practical for investigating nanoparticle haloing mechanisms, it is currently difficult to align a sphere coaxially with another sphere between smaller spherical particles $(\sim 1$ 
$\mu \mathrm{m})$, so that the initial measurements discussed in this work were conducted between a pre-attached microsphere and a flat substrate. This study focuses on the investigation of the interaction forces between a silica sphere and a silica flat surface in zirconia nanoparticle suspensions with a variable volume fraction at $\mathrm{pH} 1.5$. Zirconia nanoparticle haloing around silica spheres was observed at such $\mathrm{pH}$ value as reported in prior work. ${ }^{98}$ Different nanoparticle concentrations and sizes have been studied. The direct observation of interaction forces in a nanoparticle haloing system will provide a significant understanding of the phase behavior of colloidal suspension in the presence of charged nanoparticles.

\subsection{Materials and Experimental Method}

Materials

The silica microspheres used in force measurement are pre-attached on V-shaped silicon nitride cantilevers (NOVASCAN, Ames, IA, spring constant $\approx 0.15$ ), with diameters of $600 \mathrm{~nm}$ and $1 \mu \mathrm{m}$. A silica circular plate with root mean square surface roughness of $<2 \mathrm{~nm}$ (height of 1/16 in., diameter of 1/2 in., Quartz Scientific, Fairport Harbor, $\mathrm{OH}$ ) served as the flat substrate. This silica plate has a purity of $99.90 \%$. The zirconia nanoparticles (Nyacol Nano Technologies Inc., Ashland, MA) were supplied in suspension $(\mathrm{pH} \approx 3.5)$, with diameters of $10 \mathrm{~nm}, 50 \mathrm{~nm}$, and $100 \mathrm{~nm}$ respectively. By adding an appropriate amount of DI water, the volume fractions of zirconia suspensions were prepared to be $10^{-3}, 10^{-4}, 10^{-5}$, and $10^{-6}$ for each size. The $\mathrm{pH}$ of the suspensions was adjusted to 1.5 by adding nitric acid (Fisher Scientific, Pittsburgh, PA). No additional salt was involved in the zirconia suspension that would potentially impact the force 
measurement. The nanoparticle suspensions were then dispersed using an ultrasonic dismembrator (Fisher Scientific, Pittsburgh, PA) for about $1 \mathrm{hr}$ before use.

Methods

Zeta potentials of the silica-zirconia mixtures were measured using a 90 Plus-Zeta particle size analyzer (Brookhaven Instruments, Holtsville, NY). The binary suspensions for zeta potential measurements were prepared by adding an appropriate amount of silica microspheres (Bangs Laboratories, Inc. Fisher, IN) to the zirconia suspensions that have been prepared as described above. The $\mathrm{pH}$ value of the mixture was adjusted to 1.5 by adding nitric acid and then was sonicated for an hour. The silica microspheres had average diameters of $600 \mathrm{~nm}$ and $1 \mu \mathrm{m}$ respectively.

Force measurements between silica surfaces were made using an XE-100 AFM

(Park Systems, Santa Clara, CA) operating at a scan rate of $100 \mathrm{~nm} / \mathrm{s}$ in zirconia nanoparticle suspensions which were contained in a Petri dish. The silica plate was initially sonic cleaned for ten minutes, and then cleaned alternatively by deionized water and anhydrous ethanol three times. After that, the surface was dried in a laminar flow hood before each experiment. Four different microsphere-nanoparticle size ratios were investigated: $100(1 \mu \mathrm{m} / 10 \mathrm{~nm}), 60(600 \mathrm{~nm} / 10 \mathrm{~nm}), 20(1 \mu \mathrm{m} / 50 \mathrm{~nm})$ and $10(1 \mu \mathrm{m} / 100 \mathrm{~nm})$. For each size ratio, the direct force measurements were conducted in zirconia nanoparticle suspensions with volume fractions of $10^{-3}, 10^{-4}, 10^{-5}$, and $10^{-6}$.

\subsection{Results and discussion}

The effect of zeta potential on the stabilization was firstly investigated in this study. At a $\mathrm{pH}$ of 1.5 , the silica suspension has a negligible surface potential of $+1 \mathrm{mV}^{98}$ so that the electrostatic repulsion between the colloids could be ignored, while zirconia 
nanoparticles are highly charged at this $\mathrm{pH}$ value, and have a consistent zeta potential of $70 \mathrm{mV}$ with zirconia volume fraction varying from $10^{-6}$ to $10^{-3}$ as reported in literature ${ }^{98}$. The zeta potentials measurements were conducted in silica suspensions (volume fraction of $10^{-2}$ ) with nanoparticle volume fraction vary from $10^{-6}$ to $10^{-2}$ at $\mathrm{pH} 1.5$. The Zeta potential data is presented in Figure 3.1, showing that the effective zeta potential of the binary mixture increases with the nanoparticle concentration. When the nanoparticle concentration is as low as $10^{-6}$, the effective zeta potential is less than $20 \mathrm{mV}$, indicating an unstable state of colloidal suspension. As the nanoparticle volume fraction grows to $10^{-2}$, the zeta potential increases to $70 \mathrm{mV}$, suggesting a good stability. This zeta potential result is accordant with Tohver's original observations, which illustrated that the colloidal suspension can be stabilized by nanoparticles upon a critical nanoparticle concentration. ${ }^{98-100}$

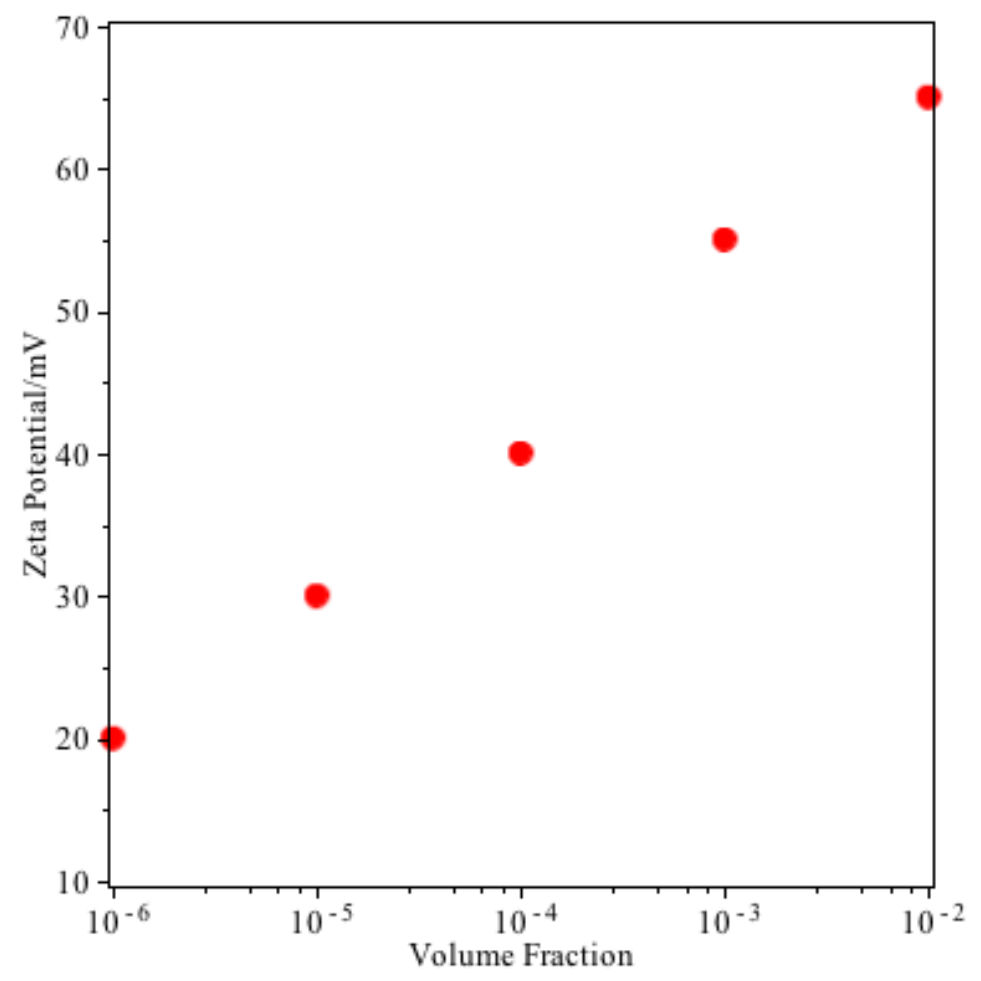


Figure 3.1 Semi-log plot of effective zeta-potential $(\xi)$ of the binary composite solution as a function of zirconia nanoparticle volume fraction in $\mathrm{pH} 1.5$ solution $\left(\Phi_{\text {silica }}=10^{-2} ; \Phi_{\text {zirconia }}=10^{-6}\right.$ $10^{-2}$, silica diameter $=1 \mu \mathrm{m}$, nano diameter $=10 \mathrm{~nm}$ ).

As seen in Figure 3.2, the interaction force between silica surfaces without the presence of nanoparticles at $\mathrm{pH} 1.5$ is dominated by attractive force at small separation (6 $\mathrm{nm})$, and it fits the theoretical van der Waals force very well. The theoretical van der Waals force is calculated using the simplified expression of Hamaker when the colloidal sizes are sufficiently large compared to the distance between them, given as: ${ }^{26}$

$$
F_{v d W}(D)=-\frac{A R}{6 D^{2}}
$$

where $A$ is the Hamaker constant of $0.8 \times 10^{-20} \mathrm{~J}$, and $R$ is the radius of the microsphere. This result confirms that the interaction force between silica surfaces at $\mathrm{pH} 1.5$ is dominated by the van der Waals attraction, and the electrostatic repulsion between them can be ignored. 


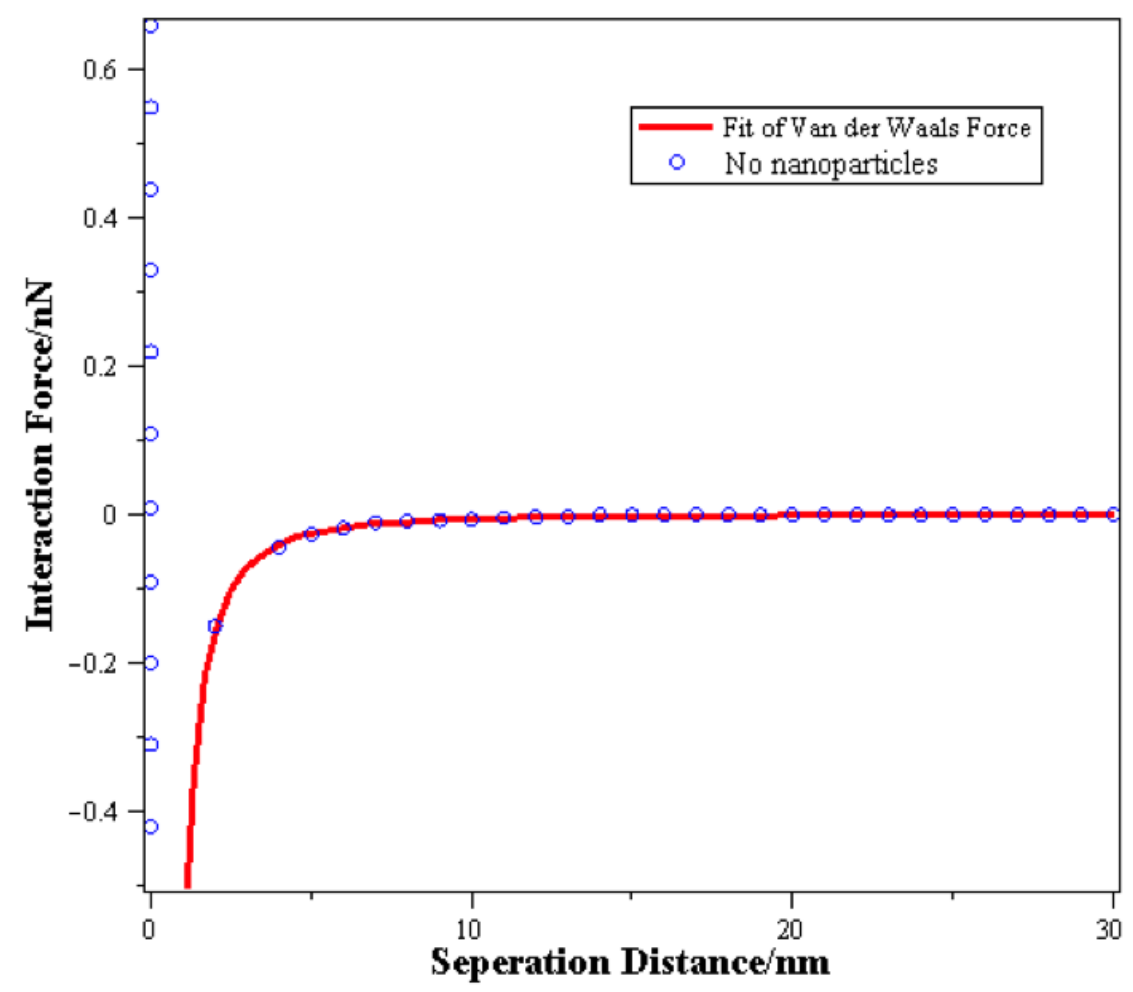

Figure 3.2 Experimental and theoretical force curves between a silica microsphere and plate without nanoparticles at $\mathrm{pH}=1.5$.

The interaction force curves between a weakly charged silica microsphere and plate in highly charged zirconia nanoparticle suspensions at different size ratios are shown in Figure 3.3. Each force curve is the average of 20 repeated curves obtained under the same conditions. It can be clearly seen that at a size ratio of 100 (Figure 3.3A), interaction force between the colloidal silica surfaces in nanoparticle suspension of $\mathrm{pH}=$ 1.5 is highly dependent on the nanoparticle volume fraction. Only the van der Waals attraction is observed when zirconia volume fraction is as low as $10^{-6}$, suggesting the state of colloidal system remains unstable. When the volume fraction increases to $10^{-5}$ the interaction force becomes repulsive, and the repulsion increases with the growing concentration. This result clearly suggests that in the presence of highly charged nanoparticles, a nanoparticle layer would form around the weakly charged silica surfaces, 
inducing a charge layer surrounding them. At low nanoparticle concentrations, the effective electrostatic repulsion is inadequate to mitigate the van der Waals attraction due to low charge density provided by nanoparticles, thus the interaction between silica surfaces is dominated by van der Waals attraction. Once the effective electrostatic repulsion is sufficient to overcome the attraction at higher nanoparticle concentrations, the colloidal system is stabilized as illustrated at volume fractions $10^{-5}-10^{-3}$. Similar results are observed at other size ratios (Figure 3.3 B, C, D). Our results are accordance with AFM force measurements made by Walz et al. in both silica-zirconia and silicapolystyrene systems. However, with a minimum nanoparticle volume fraction of $10^{-3}$, no van der Waals attraction was reported in their work.$^{97}$ 

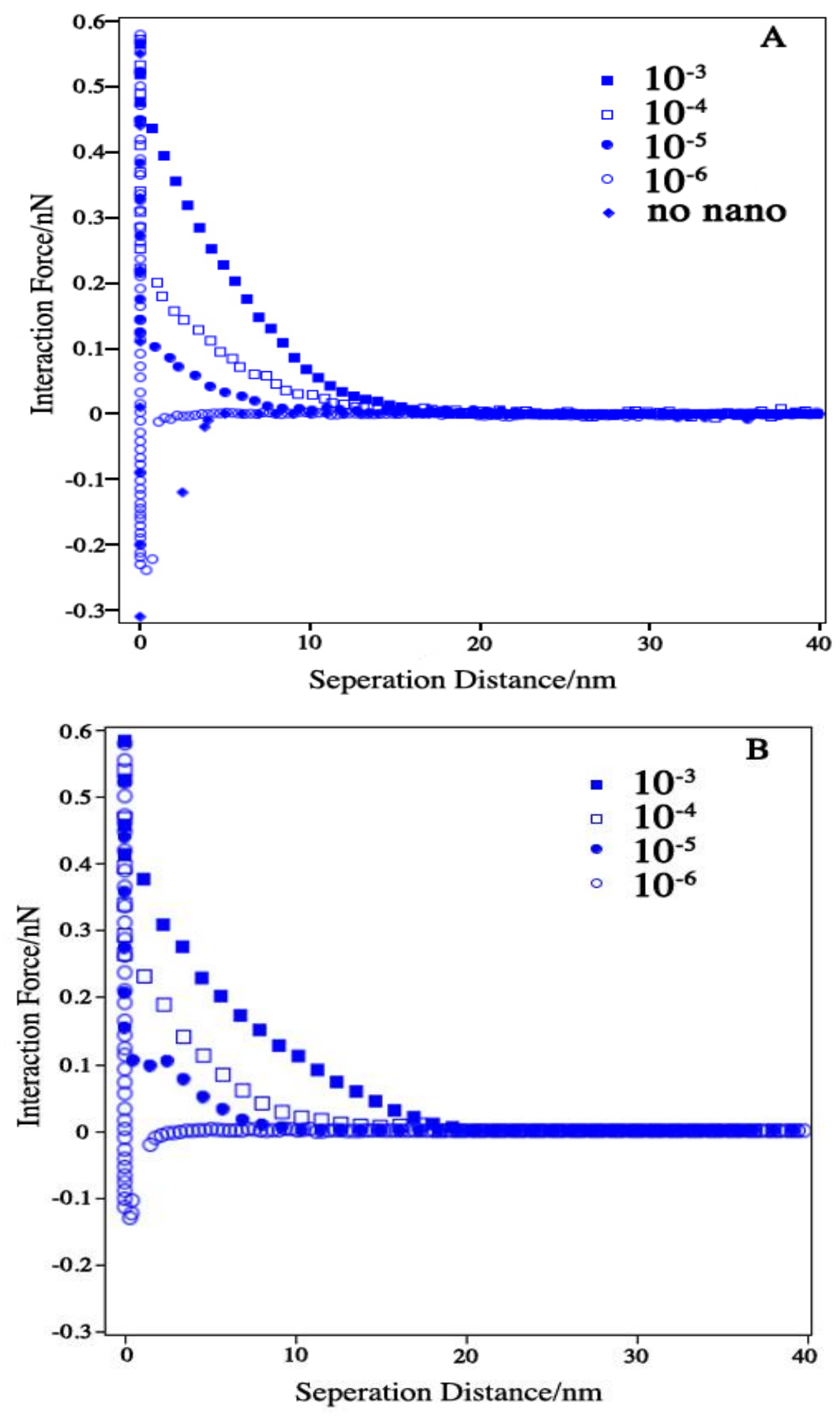

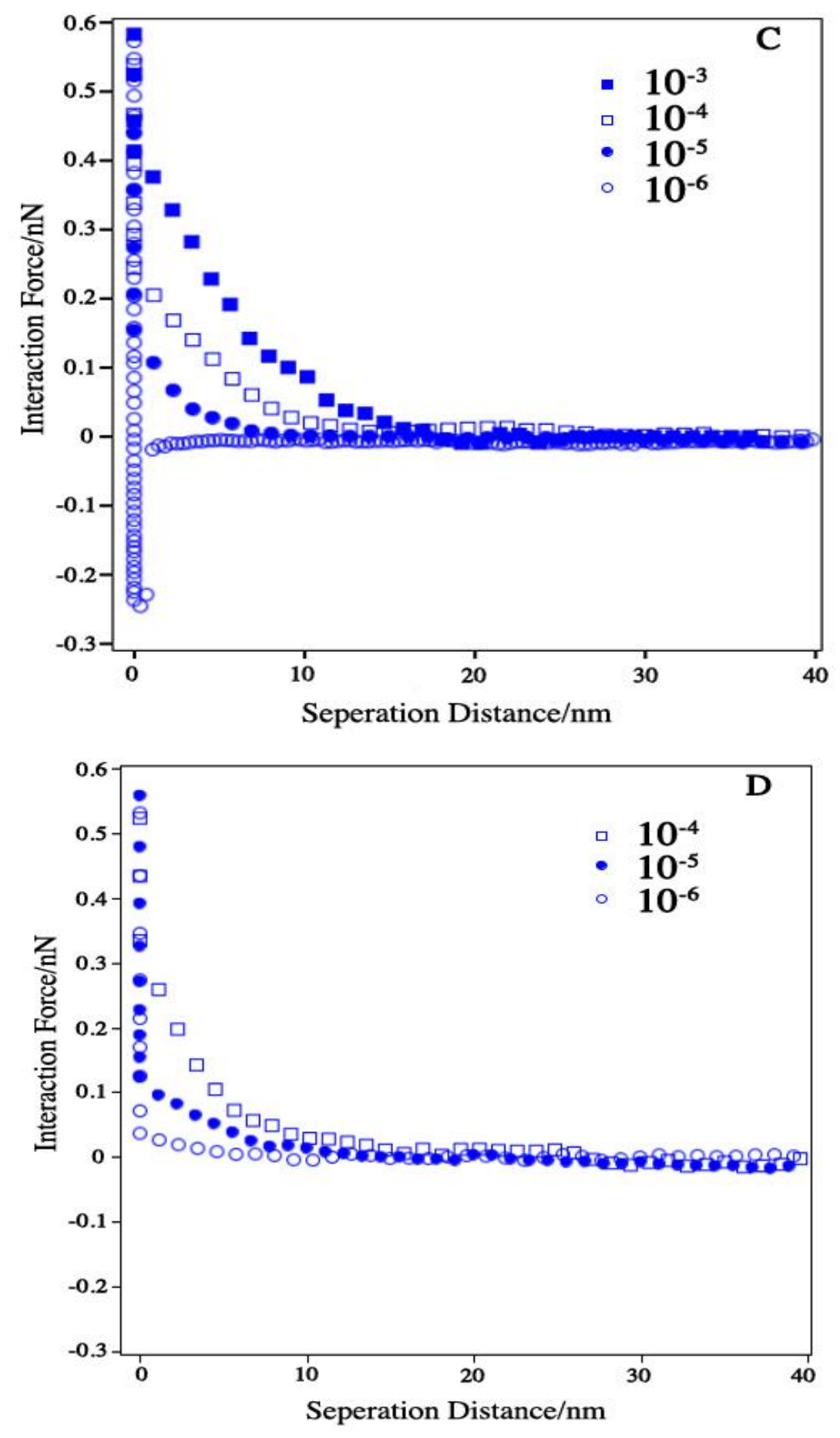

Figure 3.3 Experimental interaction forces between a silica microsphere and plate in different zirconia nanoparticle suspensions at size ratios of A) 100 B) 60 C) 20, and D) 10. 


\subsection{Conclusions}

This chapter has clearly demonstrated the transition of interaction force from a purely attraction to purely repulsion through different nanoparticle concentrations for a silica sphere-flat system at $\mathrm{pH} 1.5$ by using CP-AFM force measurements. Firstly, the experimental zeta potential results show that highly charged zirconia nanoparticles are able to enhance the stability of silica colloidal suspensions when the nanoparticle volume fraction is above $10^{-5}$. Subsequently the force measurements confirm that the interaction between silica surfaces is dominated by attraction at low nanoparticle concentration, and shifts to repulsion as zirconia volume fraction increased to $10^{-5}$. These results demonstrate that highly charged nanoparticles would gather around silica surfaces, leading to an effective charging layer surrounding them. Once the effective electrostatic repulsion induced by this charge layer is sufficient to overcome the van der Waals attraction between colloidal surfaces upon a critical nanoparticle concentration, the colloidal system will be stabilized. The states of colloidal-nanoparticle binary system indicated by the force measurement are consistent with Tohver's phase graph which shows silica suspension (with silica volume fraction lower than $10^{-2}$ ) achieves stable when nanoparticle volume fraction increases to $10^{-5} \cdot{ }^{98-100}$

These direct force measurements provide new insight to understand stabilization mechanism in nanoparticle / microsphere binary colloidal mixture. In the next chapter, a theoretical model is developed based on these experimental force curves to estimate interaction in general nanoparticle-regulated colloidal suspensions. 


\section{CHAPTER 4}

\section{MODELLING THE STABILIZATION MECHANISM OF COLLOIDAL SUSPENSION USING HIGHLY CHARGED NANOPARTICLES}

\subsection{Introduction}

As shown in the previous chapter, surface forces in nanoparticle-regulated colloidal system have been measured directly by using CP-AFM technique. In this chapter a theoretical model has been developed to estimate interaction between colloidal surfaces in such system.

Since the new stabilization of colloidal suspension named nanoparticle halo was proposed in 2001, the use of nanoparticles to alter the interaction force between larger colloidal particles have been extensively investigated. In their original work, Tohver et al. suggested that these halos could arise from strong electrostatic repulsive forces between the nanoparticles (i.e., the nanoparticles were crowded close to the microspheres by multi-particle interactions). Subsequently, Monte Carlo simulations made by Luijten and coworkers indicated that an alternative scenario was that the nanoparticles were attracted to the microspheres by either attractive electrostatic forces or weak van der Waals forces (such an attraction would likely be the dominant 
mechanism for the formation of a halo in the dilute nanoparticle limit). The result of these halos was a long-range repulsion between the weakly-charged microspheres that, theoretically, was sufficient to stabilize them against aggregation.

Exploring the interaction forces between colloidal particles has significant importance in understanding the interparticle interaction for controlling the stability of a colloidal system. By simply a sphere-sphere system to a sphere-plate system, CP-AFM measurement is able to qualify the surface interaction forces in the nanoparticle-regulated colloidal system. We are the first group that have directly measured the interaction force between two silica surfaces in the presence of highly charged nanoparticles using the CPAFM. ${ }^{112}$ The experimental force curves show that the interaction at low nanoparticle concentration is attractive, and became repulsive as the concentration increased, indicating an electrostatic repulsion between weakly charged colloidal surfaces is induced by the addition of highly charged nanoparticles. Once the effective repulsion is sufficient to overcome van der Waals attraction above a critical nanoparticle concentration, the colloidal microsphere-plate system would be prevented from aggregation. These results suggest that highly charged nanoparticles are enriched in the region around colloid surface so that build up a nanoparticle layer around it. McKee et al. obtained similar results by means of AFM as well. ${ }^{97}$ However, the contributions of the various fundamental forces such as van der Waals force, electrostatic repulsion or depletion force to the repulsion-dominated interaction still remain ambiguous. Besides, despite the studies referenced above, no one has reported how the halos vary with different nanoparticle concentrations and sizes. 
The objective of this chapter is to elucidate how charged nanoparticles impact interactions between colloidal surfaces and provide a model of broadly applicability to estimate the interactions in microsphere/nanoparticle binary systems. The development of nanoparticle halo upon varying nanoparticle concentrations has been studied as well.

\subsection{Theory and Models}

According to DLVO theory, the forces between two surfaces in a liquid can be regarded as the sum of two contributions. These are the van der Waals forces and the electrical double layer forces due to the overlapping of the electrical double layers of two neighboring particles. In our experimental system, despite the electrostatic interaction between silica surfaces is negligible because of the zero charge at $\mathrm{pH} 1.5$, an effective repulsion would be generated upon addition of highly charged nanoparticle as indicated in previous study. ${ }^{112}$ Thus, an effective electrostatic repulsion was the first consideration for this model.

\section{(1) Electrostatic Repulsion}

In this work, Chan's algorithm ${ }^{184}$ which is able to compute the electrostatic interaction across symmetric electrolytes with high precision is firstly utilized to calculate the repulsive interaction energy between a microsphere and plate. 


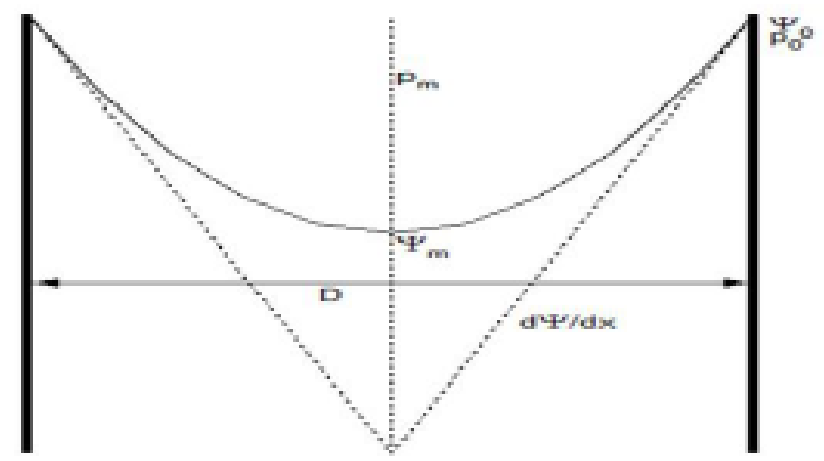

Figure 4.1 The potential profile between planar double layers.

For identical, charged plane-plane interfaces immersed in 1:1 electrolyte, the schematic of potential profile between planar double layers is shown in Figure 4.1. The identical potential and osmotic pressure at the surface of the plates is defined to be $\Psi_{0}$ and $p_{0}$. The osmotic pressure between the plates varies from point to point because of the variation in the potential $\Psi . \Psi_{m}$ and $p_{m}$ presents the potential and osmotic pressure at the midplane, respectively.

The planar Poisson-Boltzmann equation in a 1:1 electrolyte solution of number concentration $n$ is

$$
\frac{d^{2} Y}{d X^{2}}=\sinh Y
$$

where $Y=e \Psi / k T$ and $X=\kappa x$ is the scaled potential and scaled distance respectively, and $\kappa^{-1}$ is the Debye length.

At first integration yields

$$
\frac{d Y}{d X}=Q \operatorname{sgn}\left(Y_{m}\right)
$$

Where $Q$ is defined as 


$$
Q=\sqrt{2\left(\cosh Y-\cosh Y_{m}\right)}
$$

Based on the following relationship

$$
\frac{d X}{d Q}=\frac{\operatorname{sgn}(Y)}{Q}=\frac{\operatorname{sgn}\left(Y_{m}\right)}{Q}\left[\left(\frac{Q^{2}}{2}+\cosh Y_{m}\right)^{2}-1\right]^{0.5}
$$

and equation (4.2), one can derive the differential equation

$$
\frac{d X}{d Q}=\left[\left(\frac{Q^{2}}{2}+\cosh Y_{m}\right)^{2}-1\right]^{0.5}
$$

In equilibrium, where two surfaces approach one another slowly, several possible cases might occur: constant surface potential, constant surface charge and neither surface potential nor surface charge is constant. The constant charge refers to overall charge of the system and the number of electrons are held constant; the constant potential refers to the surface potential over the colloids is constant.

Equation (4.5) can be integrated by a suitable numerical technique to calculate the relationship between $\mathrm{X}$ and $\mathrm{Y}_{\mathrm{m}}$ if the value of $Q$ on the surface $\left(Q_{s}\right)$ is known.

At constant surface charge $\left(\sigma_{0}\right)$,

$$
Q_{s}=\operatorname{sgn}\left(Y_{m}\right)\left(\frac{Y}{X}\right)\left(Y=Y_{0}\right)=\operatorname{sgn}\left(Y_{m}\right) \frac{e}{k T \kappa}\left(\frac{d \Psi}{d x}\right)\left(\Psi=\Psi_{0}\right)=\left|\frac{e \sigma 0}{\epsilon k T \kappa}\right|
$$

By picking an arbitrary value in the range of $\left|Y_{m}\right|<\infty$, equation (4.5) can be numerically integrated from the midplane $Q=0$ to $Q=Q_{s}$. If there is a non-zero $\mathrm{X}$ for every $Y_{m}$, then the value of $Y_{m}$ is recorded. The corresponding non-zero $X$ value stands for half of the separation between the two plates, that is, $X=\kappa D / 2$. Therefore, a relation between the separation $D$ and $Y_{m}$ can be obtained by choosing a number of $Y_{m}$ values with $Y_{m}$ increasing to infinity.

At constant surface potential,

$$
Q_{s}=\sqrt{2\left(\cosh Y_{0}-\cosh Y_{m}\right)}
$$


Again by arbitrarily selecting a number from the range of $\left|Y_{m}\right|=\left|Y_{0}\right|$, equation (4.5) can be numerically integrated from the midplane $Q=0$ to $Q_{s}$ to obtain a relation between separation $D$ and $Y_{m}$.

The electrostatic pressure at each value of $D$ is simply calculated from the corresponding $Y_{m}$ value by

$$
p(D)=2 n k T\left(\cosh Y_{m}-1\right)
$$

The interaction free energy per unit surface area $V$ can be computed from

$$
V=\int_{D}^{\infty} p d D
$$

Besides this theoretical integration, there are several simplified approximations that can be used to roughly estimate the electrical double-layer interaction depending on suspension properties. In practice, if the separation distance between surfaces is small compared to the size of spheres but large compared to Debye length, interaction energy between two spheres with identical radius of $R$ can be calculated by using Hogg-HealyFuerstenau(HHF) approximation formula ${ }^{39}$.

$$
V^{\psi}=2 \pi R \varepsilon_{0} \varepsilon_{r} \psi_{0}^{2} \ln [1+\exp (-\kappa D)]
$$

where $\varepsilon_{0}$ is the vacuum permittivity, $\varepsilon_{\mathrm{r}}$ is the dielectric constant.

To calculate the potential between a colloidal microsphere and plate, the HHF equation is expressed as:

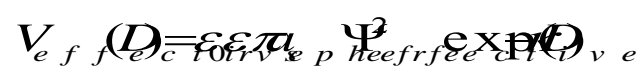

where $\mathrm{a}_{\text {sphere }}$ is the radius of the silica particle, and $\psi_{\text {effective }}$ is zeta potential of the binary mixture. 
In this work, force measurements were conducted between a silica microsphere and silica plate substrate in nanoparticle suspensions at $\mathrm{pH}$ of 1.5 . Surface charge potentials change from $20 \mathrm{mV}$ to $60 \mathrm{mV}$ at varying nanoparticle concentrations. Under these conditions, both theoretical integration and HHF approximation have been employed to estimate the electrical double-layer interactions between the microsphere and plate in the presence of charged nanoparticles. Results are shown in Figure 4.2.
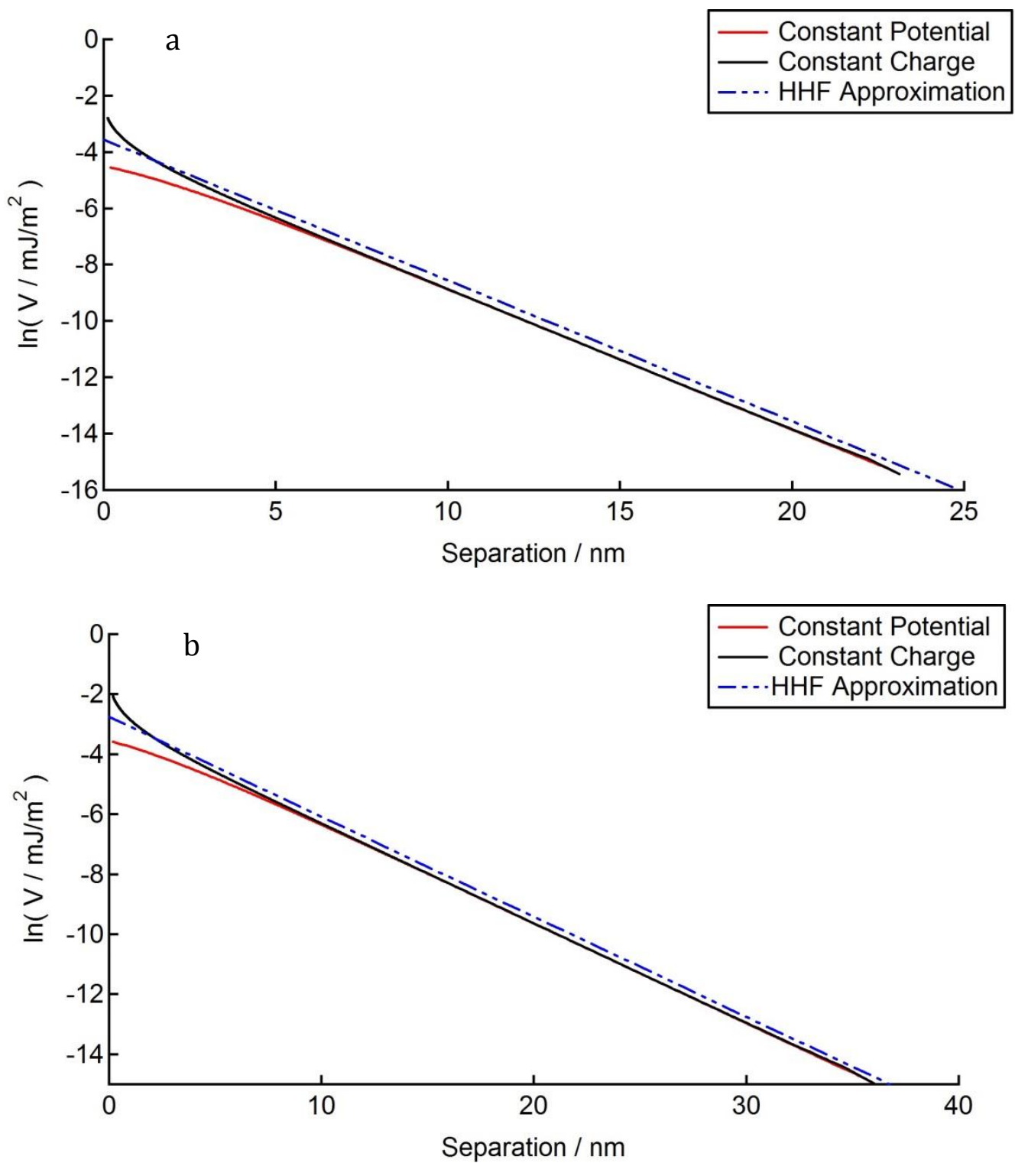

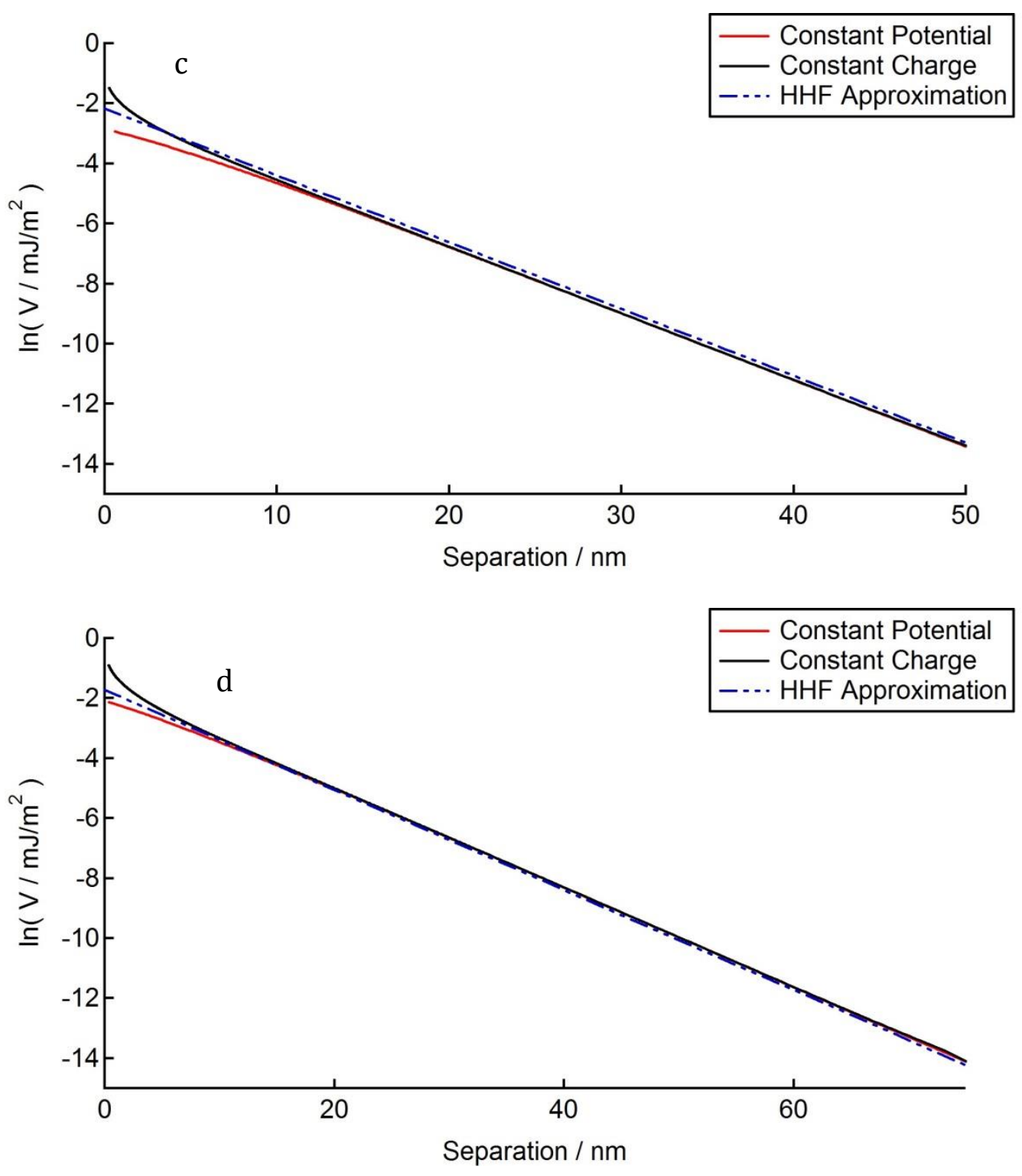

Figure 4.2 Comparison of electrostatic interaction between a sphere and flat based on theoretical integration and HHF approximation at $\mathrm{pH} 1.5$ and zeta potential of a) $20 \mathrm{mv}$, b) $30 \mathrm{mv}$, c) $40 \mathrm{mv}$ and d) $50 \mathrm{mv}$.

As shown in Figure 4.2.a, there is only a slight difference between the HHF approximated curve and theoretical integration curves at $20 \mathrm{mV}$, and this difference diminished when surface charge reached 50mv (Figure 4.2d). Besides, the forces for both cases are still in the same order of magnitude even at low potential, meaning the HHF 
approximation equation is of sufficient accuracy to evaluate the electrostatic repulsion in our experimental system. Thus, HHF formula is used to estimate the electrostatic force in our study.

The HHF approximation is well known to calculate the double layer interactions at constant surface potential between dissimilar surfaces, and has been utilized to investigate mechanisms of nanoparticle halo in several other works. ${ }^{106,107}$ We assumed the effective zeta potential was the same for both silica microsphere and plate, and a continuum assumption was made for the overlapping of the effective double layers as the charged layers associated with silica surfaces were approaching.

Considering the nature of the differential relationship between potential and interaction force, the electrostatic force equation is derived as

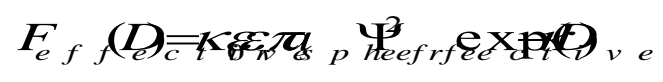

(2) van der Waals Force

Under the condition of nanoparticle halo, with a weak interaction between the halo and the colloidal particle, the van der Waals attraction between silica microsphere and plate is barely affected by zirconia nanoparticles, because these particles would be easily pushed out of the space between the approaching surfaces. Therefore, the simplified expression of Hamaker's model is adequate to estimate the van der Waals force in our model, given as: ${ }^{26}$

$$
F_{v d}(D)=-A_{D} R / 6 D^{2}
$$

where $R$ is the radius of the microsphere ( $\mathrm{a}_{\text {sphere }}$ ) and $A_{D}$ is the Hamaker constant of $0.8 \times 10^{-20} \mathrm{~J}^{21}$

(3) Depletion Force 
When a sphere approaches a plate in a nanoparticle suspension at low volume fraction, an attractive depletion force may begin to evolve. The nanoparticles cannot penetrate into the gap between the two surfaces, creating a negative osmotic pressure leading to an outflow of solvent which leads to a further attraction between the surfaces. The depletion force between a microsphere and plate in nanoparticle solution could be approximated using Piech's equation ${ }^{76}$, written as:

$$
F_{\text {depletion }}(D)=\left\{\begin{array}{c}
-2 \rho_{\infty} k T \pi\left[a^{2}+2 a R-R D-\frac{D^{2}}{4}\right] \text { for } 0 \leq D \leq 2 a \\
0 \quad \text { for } \quad D>2 a
\end{array}\right.
$$

where $a$ is the nanoparticle size, $\rho_{\infty}$ is the bulk number density, and $k T$ is equivalent to $4.11 \times 10^{-21} \mathrm{~J}$ at room temperature.

However, it was found that the effect of the depletion force could be ignored due to the fact that $F_{d e p}$ has a value of $5.8 \times 10^{-5} \mathrm{nN}$ at nanoparticle volume fraction of $10^{-3}$ which is much smaller than the magnitude of the measured total interaction force $\left(\sim 10^{-}\right.$ $\left.{ }^{1} \mathrm{nN}\right)$. Therefore, depletion force is ignored in this model.

(4) The Debye Length Fitting Model

Because the depletion force from the nanoparticles is negligible even at the highest experimental volume fraction $\left(10^{-3}\right)$, the effect of the depletion force can be ignored in this study. As a result, only the van der Walls attraction and electrostatic repulsion are taken into account for the total interaction calculation.

$$
F_{\text {total }}=F_{v d W}+F_{\text {effective }}
$$

After substituting Eq. (4.12) and (4.13) into Eq. (4.15), we derived the total interaction equation thusly:

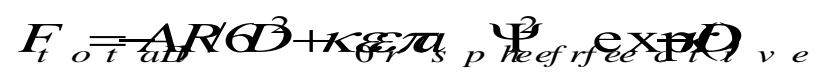


It should be noted that the term $\kappa$ in Eq. (4.16) stands for the effective Debye length of the colloid-nanoparticle mixture. Under the condition of nanoparticle halo, highly charged nanoparticles would segregate around each negligibly charged colloidal surface, forming a loose nanoparticle layer located a small distance away from the

colloidal surface. ${ }^{98-100}$ This means the gap between the microsphere surface and its effective charge plane is affected by the separation between the microsphere and nanoparticle halo as well as the nanoparticle diameter. As a result, it is impossible to calculate the thickness of the charge layer using the regular Debye length equation. Therefore, with $\kappa$ regarded as a fitting parameter, we named the total interaction force in eq.(4.16) the Debye Length Fitting Model (DLFM).

\subsection{Results and discussion}

\section{(1) Force Measurements}

The interaction force curves between a weakly charged silica microsphere and plate in highly charged zirconia nanoparticle suspensions at different size ratios are shown in Figure 4.3. Each force curve is the average of 20 repeated curves obtained under the same conditions. It can be clearly seen that at a size ratio of 100 (Figure 4.3A), interaction force between the colloidal silica surfaces in nanoparticle suspension of $\mathrm{pH}=$ 1.5 is highly dependent on the nanoparticle volume fraction, which is consistent with previous results obtained at a size ratio of 60 (Figure 4.3.B). Only the van der Waals attraction is observed when zirconia volume fraction is as low as $10^{6}$, suggesting the state of colloidal system remains unstable. When the volume fraction increases to $10^{-5}$ the interaction force becomes repulsive, and the repulsion increases with the growing concentration. This result clearly suggests that in the presence of highly charged 
nanoparticles, a nanoparticle layer forms around the weakly charged silica surfaces, leading an electrostatic repulsion between them. Similar results are observed at other size ratios (Figure 4.3.C, D).
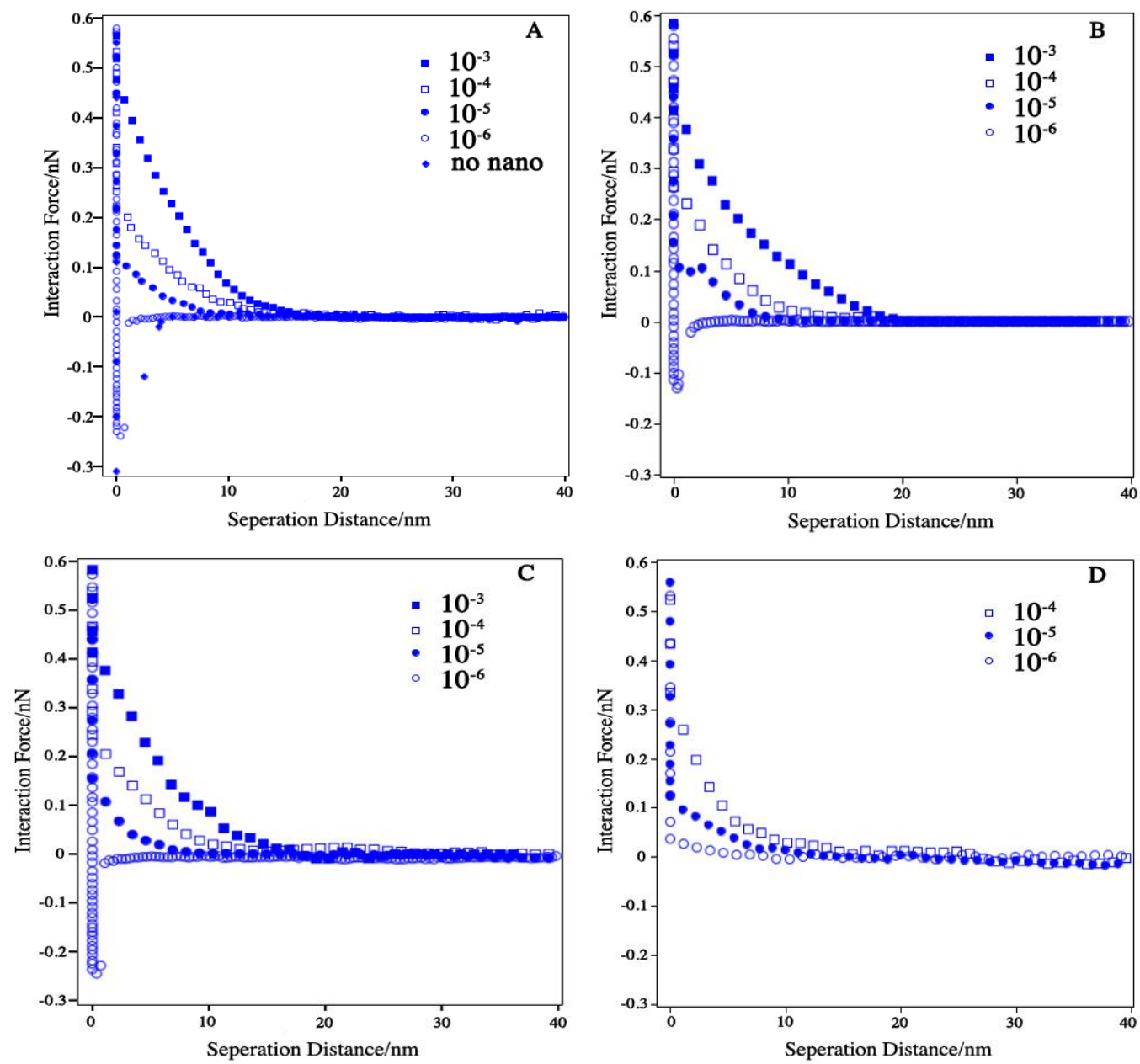

Figure 4.3 Experimental interaction forces between a silica microsphere and plate in different zirconia nanoparticle suspensions at size ratios of A) 100 B) 60 C) 20, and D) 10.

\section{(2) Fitting Results}

We substituted the measured zeta potential values into Eq. (4.16) and then adjusted the value of effective Debye length until the theoretical and experimental force 
curves were matched. The final fitting results at different size ratios are graphed in Figure 4.4, with the solid lines representing the fitting curve.
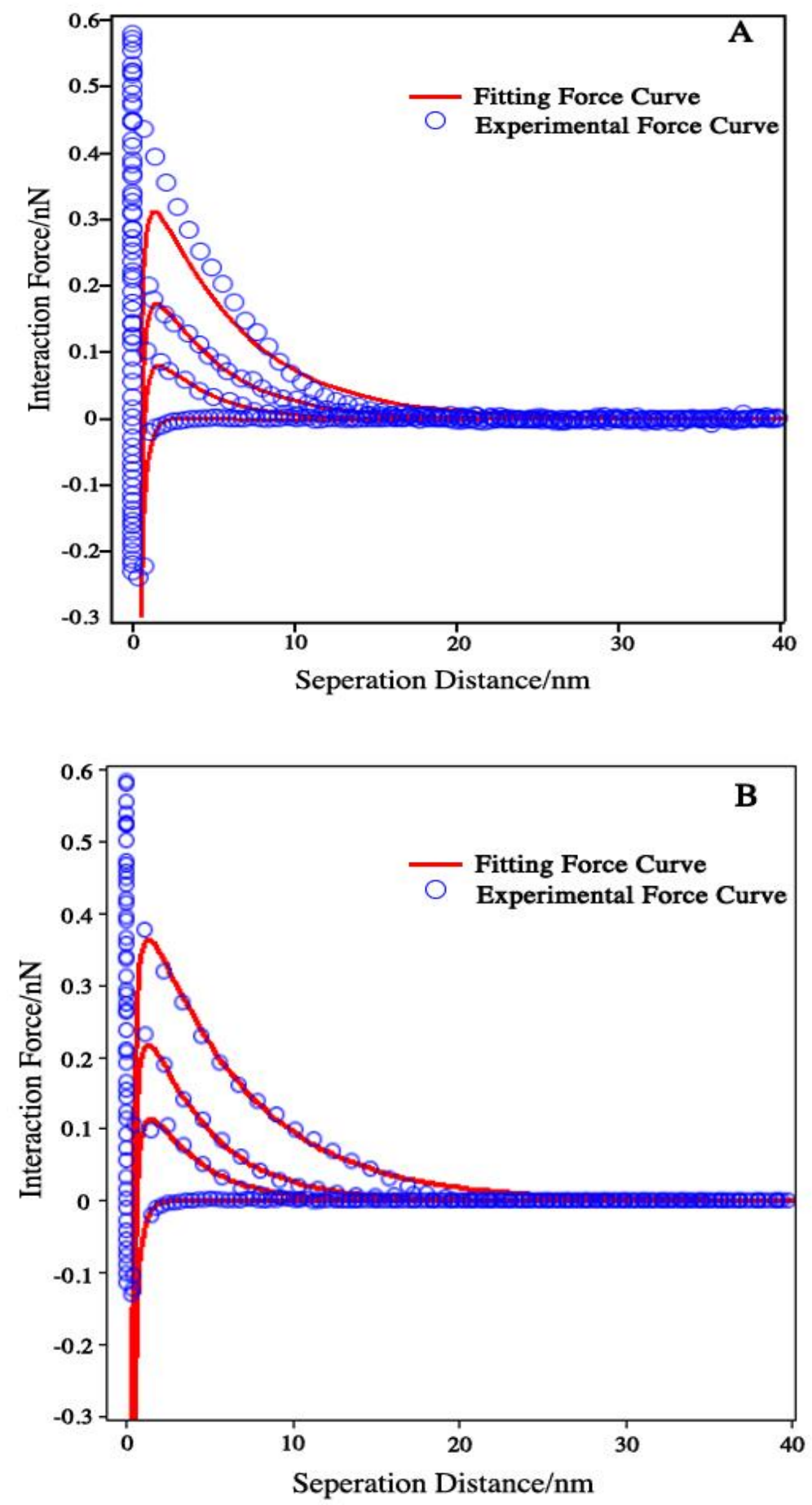


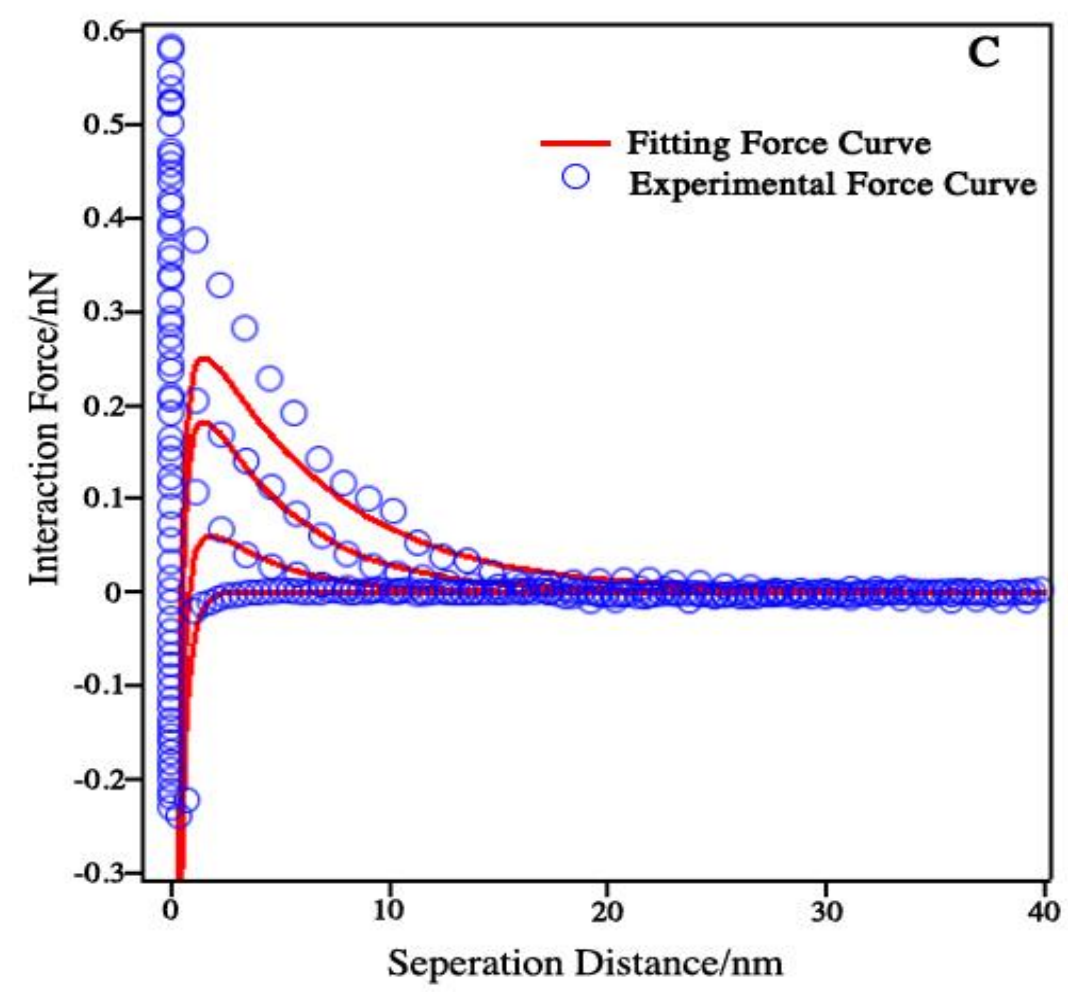

Figure 4.4 Plots of force vs. separation measured by AFM in nanoparticle suspensions with varying volume fraction (blue circles) at $\mathrm{pH}=1.5$, and the theoretical force curves calculated by the fitting model using an appropriate effective Debye length (red solid lines). Size ratios: A) 100, B) 60, C) 20.

By choosing appropriate values for effective Debye length, the DLFM fit the experimental forces very well at size ratios of 100, 60 and 20, except for small deviations appeared at volume fraction of $10^{-3}$ at size ratios of 100 and 20 . These good fitting results indicate DLFM is generally applicable to estimate the interactions in nanoparticle-halo system. According to DLFM, the total interaction force between two silica surfaces in presence of highly charged zirconia nanoparticles is mainly composed of a van der Waals attraction and an electrostatic repulsion. Once the electrostatic repulsion produced by a highly charged nanoparticle layer is sufficiently strong to overcome the van der Waals 
attraction, silica colloids will be prevented from aggregating. One possible reason for the deviation observed at volume fraction of $10^{-3}$ is the invalidation of van der Waals formula used in DLFM at such a concentration. As mentioned previously, DLFM assumed that the additional nanoparticles haloed around silica surfaces, so that the effect of charged nanoparticles on van der Waals attraction between silica surfaces was negligible due to a weak interaction between the silica sphere and the zirconia halo. This assumption works well at low volume fractions $\left(10^{-6}\right.$ to $\left.10^{-4}\right)$, suggesting nanoparticle haloing exists under these conditions. However, at higher concentrations, it's quite possible that the nanoparticle deposition becomes significant enough to alter the attraction between the absorbent surfaces. The Walz's group has recently reported that strong adsorption was observed in their nanoparticle-regulated binary system. ${ }^{181}$ In that case, multi-particle interactions (i.e., attractions between nanoparticle-nanoparticle, nanoparticlemicrosphere) should be taken into consideration when calculating van der Waals attraction. The effect of adsorption on nanoparticle haloing is elucidated in the next chapter. .

Table 4.1. Effective Debye Lengths at Varying

Nanoparticle Suspensions Determined by Fitting Model

\begin{tabular}{cllll}
\hline & \multicolumn{4}{c}{ Volume Fraction } \\
\cline { 2 - 5 } Size Ratio & $10^{-6}$ & $10^{-5}$ & $10^{-4}$ & $10^{-3}$ \\
\hline 100 & $2 \mathrm{~nm}$ & $3.3 \mathrm{~nm}$ & $4.2 \mathrm{~nm}$ & \\
$60^{1}$ & $2.5 \mathrm{n}$ & $3 \mathrm{~nm}$ & $4 \mathrm{~nm}$ & $6.5 \mathrm{~nm}$ \\
20 & $2 \mathrm{~nm}$ & $2.5 \mathrm{~nm}$ & $4 \mathrm{~nm}$ & \\
\hline
\end{tabular}

Final fitting values of the effective Debye length $\left(D_{\text {effective }}\right)$ are summarized in Table 4.1. At a size ratio of $100, D_{\text {effective }}$ were found to be $2 \mathrm{~nm}\left(10^{-6}\right), 3.3 \mathrm{~nm}\left(10^{-5}\right)$, and 
$4.2 \mathrm{~nm}\left(10^{-4}\right)$, which show a successive increase with increasing nanoparticle concentration. At $\mathrm{pH}$ 1.5, the effective Debye lengths found in our silica-zirconia systems are larger than those in a pure nitric acid solution $(\sim 1.7 \mathrm{~nm})$. This result indicates that nanoparticles segregate to regions near colloidal surface, leading to a non-zero distance between nanoparticle layer and silica microsphere which broadens the effective Debye length of the complex fluid. Based on the fact that $D_{\text {effective }}$ increases with the nanoparticle concentration, two scenarios can be used to explain how the nanoparticle halo changes with the nanoparticle concentration. One explanation is, as the number of nanoparticles increases, the distance between nanoparticle layer and silica sphere $(d)$ becomes larger in order to minimize the interaction between nanoparticles coexisting in one halo, leading to a slight shift in the plane of charge, and the effective Debye length is increased as a result. This process is schematically described in Figure 4.5a. One alternative is that $d$ is relatively constant as the nanoparticle concentration increases; it is the increasing nanoparticle density in a halo that attribute to the expanded charge layer (Figure 4.5b). In other words, when the number of nanoparticles involving in one halo increases, the average thickness of the nanoparticle layer will increase, and so does the effective charge layer. However, as suggested by other studies that nanoparticles are strongly adsorbed to colloidal surfaces at high nanoparticle concentrations, the nanoparticle layer should not grow further from the colloid with the increasing nanoparticle concentration. Therefore, realistically the radius of nanoparticle halo will not change much with nanoparticle concentrations, but the charge layer built up by the halo will. This process is illustrated in Figure 4.5b. 



$$
\Phi=10^{-5}
$$

$$
\Phi=10^{-4}
$$

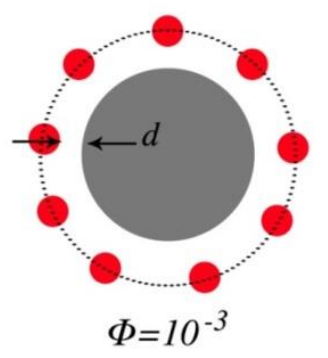

Zirconia Nanoparticles

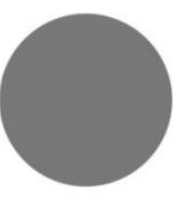

Silica Microsphere

d: distance between nanoparticle halo and silica sphere.

b

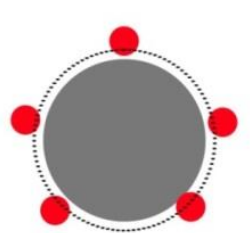

$\Phi=10^{-5}$

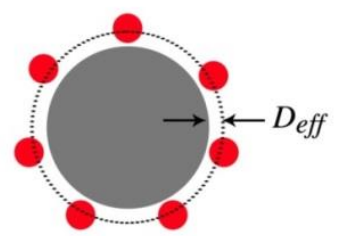

$\Phi=10^{-4}$

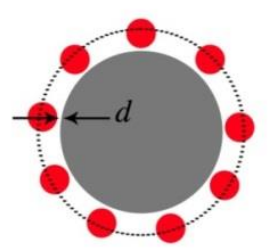

- Zirconia Nanoparticles

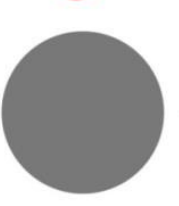

Silica Microsphere

$$
\Phi=10^{-3}
$$

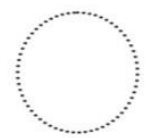

Charge Layer

$d$ : distance between nanoparticle halo and silica sphere.

Figure 4.5 Schematic description of the effect of the nanoparticle volume fraction on the gap between silica sphere and zirconia nanoparticle. 
DLFM fits the experimental data very well at the size ratios of 100,60 , and 20 , indicating its good applicability in estimating interactions in nanoparticle-haloing systems. However, when the size ratio decreased to $10(1 \mu \mathrm{m} / 100 \mathrm{~nm})$, DLFM failed to

fit the experimental curves over the entire range of nanoparticle concentrations from $10^{-6}$ to $10^{-3}$ no matter how the effective Debye length was changed, suggesting that there is no nanoparticle haloing formation at such a low size asymmetry. This is not surprising, because when the nanoparticle and microsphere size are comparable, the continuum assumption made in DLFM becomes invalid, and the nanoparticle-colloid mixture could be taken as a system of differently charged microspheres with similar size. An analytic integral equation theory has been developed by other researchers to calculate the interaction under these conditions. ${ }^{182,183 .}$

\subsection{Conclusions}

In summary, we have measured the interaction forces between a weakly charged silica sphere and plate in highly charged zirconia nanoparticle solutions at varying concentrations and size asymmetries. Upon reaching a critical low volume fraction $\left(10^{-5}\right)$, highly charged nanoparticles are able to prevent aggregation of colloidal particles by inducing an effective electrostatic repulsion. Based on the experimental data, the Debye length fitting model has been developed to generally estimate the interaction force between neutral colloidal microspheres surrounded by highly charged nanoparticles. The DLFM suggests: 1) the interaction between microspheres in the presence of nanoparticles is mainly composed of a van der Waals attraction and an electrostatic repulsion; 2) there is a non-zero distance between nanoparticle layer and colloidal surface, and the effect of nanoparticle adsorption on the interaction force between colloidal surfaces can be ignored 
at low volume fractions $\left(10^{-6}\right.$ to $\left.10^{-4}\right)$. Additionally, the fitting result of effective Debye length suggests the gap between colloidal surface and surrounding charge layer increases with increasing nanoparticle concentration.

These results illustrate that the stabilization regulated by charged nanoparticle can be well explained by the formation of nanoparticle halo which is loosely associated with colloidal surfaces to mitigate van der Waals forces that drives aggregation. This halo stabilization mechanism may be of particularly high value in applications where steric stabilization using adsorbed species may unfavorably change the particle size or may interfere with availability or reactivity of the surface.

In the next chapter, study is extended to colloidal-nanoparticle system in higher nanoparticle concentrations. If nanoparticle concentration is significantly high, either depletion force or nanoparticle adsorption may become obvious to alter interaction force between colloidal surfaces, leading to the original DLMF developed on the assumption of nanoparticle halo invalid. Therefore, more interactions are taken into consideration in the following chapter. 


\section{CHAPTER 5}

\section{TUNING THE STABILZATION MECHANISMES OF NANOPARTICLE-REGULATED COMPLEX FLUIDS}

\subsection{Introduction}

The use of nanoparticles to alter the interaction force between larger colloidal particles has been investigated for decades and continues to be a topic of interest. The appealing feature of the nanoparticle-regulated colloidal suspensions is the ability to finetune the range and magnitude of the interaction by controlling either concentration or size of the nanoparticles. In the early 1950s, Asakura and Oosawa showed theoretically that the addition of small, non-adsorbing spheres to a solution of larger spheres could produce an attractive force between the larger particles - the well-known depletion force, and derived a simple algebraic expression for the interaction between two hard spherical particles in a solution of non-adsorbing hard spherical nanoparticles that was based on the increase in free volume available to the nanoparticles upon close approach of the two

particles. ${ }^{70,71}$ Since their initial work, the effect of depletion force has been studied in great detail, both computationally and experimentally. However, in the majority of the studies the assumption has been that the nanoparticles and microspheres are mutually 
repulsive, such as hard sphere or electrostatic repulsions arising from surface charges. Such repulsion leads to negative adsorption of the nanoparticles onto the surfaces.

In 2001, Tohver et al. proposed the idea of nanoparticle halos that can arise in systems of mutually but weakly repulsive microparticle/nanoparticle dispersions. ${ }^{98,99}$ The specific system was larger particles with a very low surface charge in solution with nanoparticles that have a surface charge of the same sign but of much greater magnitude. The colloidal suspension was found to be stabilized by using zirconia nanoparticles within a critical nanoparticle concentration range. They attributed the stabilization mechanism to nanoparticle haloing which is a non-adsorbing nanoparticle layer surrounding colloidal particles that leads to an effective electrostatic repulsion between colloids to mitigate the inherent van der Waals attraction. Their follow-up study proved that zirconia nanoparticles were enriched near the surface of colloidal silica and the distance between colloid and the loosely associated nanoparticle layer was approximately equal to the suspension's Debye length. Subsequently, by using Monte Carlo simulations, Liu and coworkers showed that the formation of halo was caused by a weak attraction between a colloidal microsphere and nanoparticle at low concentrations $\left(\leq 10^{-4}\right) \cdot{ }^{106,107}$ Besides, the previous chapters have shown our CP-AFM measurement of interactions between weakly charged colloidal surfaces in the presence of highly charged nanoparticles. It is found that charged nanoparticle are able to induce a charged layer over the negligible charged colloidal surface, thus improving the stability of colloidal suspensions. And the modelling study indicates the nanoparticles segregated to regions near colloidal particles without direct adsorption onto them from volume fraction of $10^{-6}$ to $10^{-4}$. 
Since this stabilization method does not rely on adsorption, it is specifically suitable in applications where using adsorbed species may hinder reactivity or availability of the surface such as in colloidal surface functionalization and ceramics processing.

Ji et al. also investigated the use of charged nanoparticle to manipulate interaction between neutral colloids. ${ }^{181}$ However, instead of nanoparticle halo, they proposed that the stabilization of binary suspension is caused by sufficient deposition of nanoparticles onto the colloids, leading to an increase in the effective charge density on the colloidal surfaces, and thereby enhancing the electrostatic repulsion between them. This increased repulsion did not vanish upon flushing the nanoparticles out of the system, indicating a strong nanoparticle adsorption. This result challenges the potential use of highly charged nanoparticle as a tool to reversibly tailoring colloidal stability. However, their experiment focused on relatively high nanoparticle volume fractions $\left(\geq 10^{-3}\right)$.

These previous works assumed the mechanisms of nanoparticle haloing and adsorption were mutually exclusive and focused on specific, but largely distinct, nanoparticle concentrations. In this study, we find that these two mechanisms work across a continuum to regulate the stability of colloidal suspensions over increasing nanoparticle concentrations. Firstly, AFM force measurements show that highly charged zirconia nanoparticles built up an electrostatic repulsion between negligible charged silica surfaces, thereby preventing them from aggregation at zirconia nanoparticle volume fractions from $10^{-5}$ to $10^{-2}$. The follow-up adsorption tests and force modeling indicate that minor adsorption of nanoparticles is expected at volume fractions of $10^{-5}-10^{-3}$, whereas nanoparticles are found to be strongly adsorbed onto colloidal surfaces from $10^{-3}$ to $10^{-2}$. Based on these results, we propose that 1) the fundamental mechanism of 
nanoparticle-regulated stabilization is "nanoparticle haloing" at low nanoparticle concentrations, and transits to "adsorption" at high concentrations; 2) there is a transition region within which the stabilization can be influenced by both nanoparticle haloing and adsorption. This transition was observed around a nanoparticle volume fraction of $10^{-3}$ in our experiments. Our study suggests that when using highly charged nanoparticles to stabilize a weakly charged colloidal suspension, the reversibility of stabilization and accessibility of colloidal surfaces can be controlled by tuning the nanoparticle concentration.

\subsection{Materials and Methods}

\subsection{Materials}

Deionized water was obtained from a Mili-Q system (EMD Millipore, Billerica, MA), with a resistivity of $18 \mathrm{M} \Omega \cdot \mathrm{cm}$. The zirconia nanoparticles (Nyacol Nano Technologies Inc., Ashland, MA) were supplied in colloidal suspensions $(\mathrm{pH} \approx 3.5)$, with manufacturer reported diameter of $10 \mathrm{~nm}$. The average diameter of the hydrous zirconia nanoparticle was $9 \mathrm{~nm}: 12 \%$ are $<5 \mathrm{~nm} ; 72 \%$ are between $5 \mathrm{~nm}$ and $10 \mathrm{~nm} ; 16 \%$ are $>10$ $\mathrm{nm}$. By adding an appropriate amount of DI water, the volume fractions of zirconia suspensions were prepared to be $10^{-2}, 10^{-3}, 10^{-4}, 10^{-5}$ and $10^{-6}$. The $\mathrm{pH}$ of suspension was then adjusted to 1.5 by adding nitric acid (Fisher Scientific, Pittsburgh, PA). An AB15 PLUS pH meter (Fisher Scientific, Pittsburgh, PA) was used to measure the $\mathrm{pH}$ values. Suspensions were dispersed using ultrasonic (Fisher Scientific, Pittsburgh, PA) for about $1 \mathrm{hr}$ before use.

Force measurements were conducted by an XE-100 AFM (Park Systems, Santa Clara, CA). The silica microspheres were pre-attached on V-shaped silicon nitride 
cantilevers (NOVASCAN, Ames, IA, spring constant $\approx 0.15 \mathrm{~N} / \mathrm{m}$ ), with diameters of 600 $\mathrm{nm}, 1 \mu \mathrm{m}$. A silica circular plate with root mean square surface roughness of $<2 \mathrm{~nm}$ (height of 1/16 in., diameter of 1/2 in., Quartz Scientific, Fairport Harbor, OH) served as the flat substrate.

\subsection{Methods}

The binary suspensions for zeta potential measurements were prepared by adding an appropriate amount of silica microspheres (Bangs Laboratories, Inc. Fisher, IN) to the zirconia suspensions that have been prepared above. The silica microspheres had average diameters of $600 \mathrm{~nm}$ and $1 \mu \mathrm{m}$. $\mathrm{pH}$ value of the mixture was adjusted to 1.5 by adding nitric acid and then was sonicated for an hour. Both zeta potentials of the silica-zirconia mixtures and particle sizes were analyzed by a 90 Plus-Zeta particle size analyzer (Brookhaven Instruments, Holtsville, NY).

Force measurements were made between a silica microsphere and plate by an XE100 AFM (Park Systems, Santa Clara, CA) at a scan rate of $100 \mathrm{~nm} / \mathrm{s}$ in zirconia nanoparticle suspensions that were contained in a Petri dish. The silica plate was initially sonic cleaned for ten minutes, and then cleaned alternatively by DI water and anhydrous ethanol three times. After that, the surface was dried in a laminar flow hood before each experiment. Two microsphere-nanoparticle size ratios were investigated in this study: $100(1000 \mathrm{~nm}$ vs. $9 \mathrm{~nm})$ and $60(600 \mathrm{~nm}$ vs. $9 \mathrm{~nm})$.

A scanning electron microscope (SEM) (FEI Nova 600) was used to measure the nanoparticle adsorption on silica plate by scanning the surface. Silica plates were initially sonic cleaned for ten minutes, and then cleaned alternatively by DI water and anhydrous ethanol three times. Different volume fractions of nanoparticle suspensions at $\mathrm{pH}$ of 1.5 
were prepared as above, and then sonicated for about $1 \mathrm{hr}$. The cleaned silica plates were fully placed in nanoparticle suspensions for $30 \mathrm{~min}$. After taken out of the solution, the silica plate was gently rinsed in DI water that was pre-adjusted to the same $\mathrm{pH}$ value to remove the non-adsorbed nanoparticles from the plate surfaces. Then the plate was dried in air and taken to the SEM measurement.

\subsection{Results and discussion}

The interaction force curves between a weakly charged silica microsphere and plate in highly charged zirconia nanoparticle suspensions at different size ratios are shown in Figure 5.1. Each force curve is the average of 20 repeated force curves obtained from different locations under the same conditions. As seen in Figure 1a, at size ratio of 100 , the interaction between a silica microsphere and plate in nanoparticle suspension of $\mathrm{pH}=1.5$ is sensitively dependent on the nanoparticle volume fraction. Only the attractive force is observed when the zirconia volume fraction is as low as $10^{-6}$. But the interaction force becomes completely repulsive as nanoparticle volume fraction increased to $10^{-5}$, and the repulsion becomes stronger as the nanoparticle volume fraction increases. These force profiles indicate that colloidal suspensions are stabilized within volume fraction range of $10^{-5}$ and $10^{-2}$ due to the domination of repulsion between the colloidal surfaces. A similar result is observed at a size ratio of 60 (Figure 5.1b). According to DLVO theory, which defines the interaction between charged surfaces in an aqueous solution as a combination of the van der Waals attraction and an electrostatic repulsion, the interaction between silica particle and plate is dominated by van der Waals attraction due to a negligible charge on their surfaces in the absence of nanoparticles. The tendencies of increasing repulsion demonstrate that highly charged nanoparticles will induce an 
electrostatic repulsion between silica surfaces, and it becomes stronger as the number of nanoparticles that are surrounding the colloidal surfaces increases.
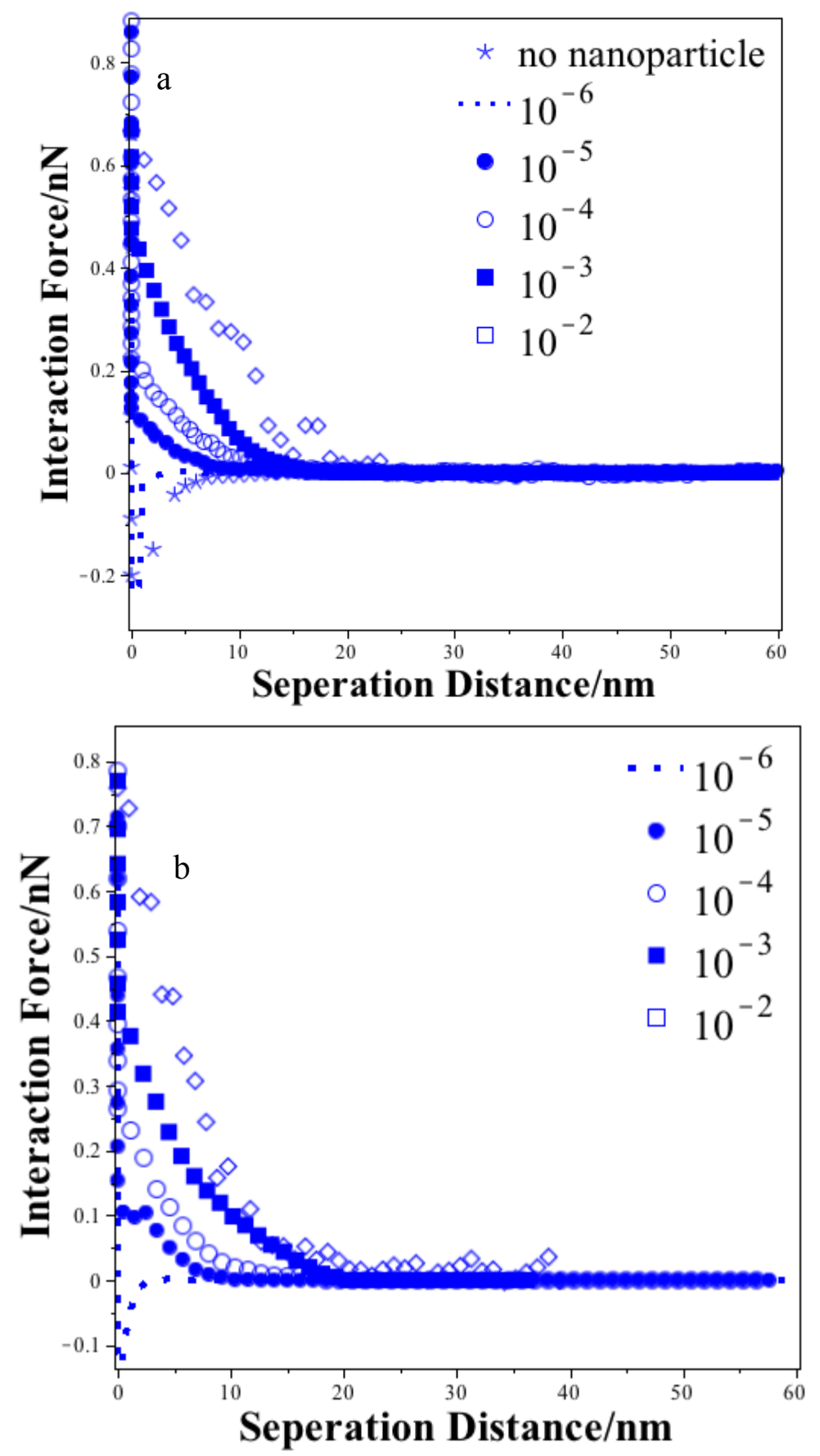

Figure 5.1 Force profiles between a silica microsphere and plate in varying volume fractions of zirconia nanoparticles at size ratio of (a) 100; (b) 60. 
In our previous work, a Debye length fitting model (DLFM) was developed to simply estimate the interaction between colloidal surfaces in the presence of nanoparticles. In this model the total interaction is expressed as the combination of van der Waals force, electric repulsive force and depletion force: $F_{\text {total }}(D)=F_{v d W}+$ $F_{\text {electro }}+F_{\text {depletion }}(5.1)$. The approximated equation for each of the forces is expressed as follows:

$$
\begin{gathered}
F_{v d W}(D)=-\frac{A R}{6 D^{2}} \\
F_{\text {electro }}(D)=-\kappa R \varepsilon_{0} \varepsilon_{r} \pi \Psi_{\text {effective }}^{2} \exp (-\kappa D) \\
F_{\text {depletion }}(D)=\left\{\begin{array}{rr}
-2 \rho_{\infty} k T \pi\left[a^{2}+2 a R-R D-\frac{D^{2}}{4}\right] \\
\text { for } 0 \leq D \leq 2 a \\
0 \quad \text { for } \quad D>2 a
\end{array}\right.
\end{gathered}
$$

where $D$ is the separation distance of the closest approach between the sphere and the plate, $R$ is the radius of the microsphere and $A$ is the Hamaker constant of $0.8 \times 10^{-20} \mathrm{~J}{ }^{127}$ $\varepsilon_{0}$ is the vacuum permittivity, $\varepsilon_{\mathrm{r}}$ is the dielectric constant, $\kappa$ is reciprocal of the Debye length, $\psi_{\text {effective }}$ is zeta potential of the binary mixture, $a$ is the nanoparticle size, $\rho_{\infty}$ is the bulk number density, and $k T$ is equivalent to $4.11 \times 10^{-21} \mathrm{~J}$ at room temperature. The van der Waals attraction between a silica sphere and plate is calculated using the simplified expression of Hamaker when the colloidal sizes are sufficiently large compared to the distance between them. ${ }^{26}$ The electrostatic repulsion is calculated using the Hogg-HealyFuerstenau (HHF) formula ${ }^{39}$, which is well known to calculate the double layer interactions at constant surface potential between dissimilar surfaces and has been utilized to study mechanisms of nanoparticle halo in several other works. ${ }^{106,}{ }^{107} \mathrm{We}$ assume that the effective zeta potential is the same for both silica sphere and plate, and a continuum assumption is made for the overlapping of effective double layers as silica 
sphere approaching the plate. The depletion force is estimated using Piech and Walz's approximation. ${ }^{76}$ Despite the effect of depletion force was neglected in our original work of DLFM, the depletion force is taken into account in this study due to the high nanoparticle concentration dealt with herein.

It should be noted that the term $\kappa$ in Eq. (5.3) stands for the effective Debye length of the colloid-nanoparticle mixture. Under the conditions of the nanoparticle halo, highly charged nanoparticles would segregate to each negligibly charged colloidal surface, forming a loose nanoparticle layer located a small distance away from the colloidal surface. This means the gap between the microsphere surface and its effective charge plane is affected by the separation between the microsphere and nanoparticle halo as well as the nanoparticle diameter. As a result, it is impossible to calculate the thickness of the charge layer using the regular Debye length equation. Therefore, $\kappa$ is taken as a variable and is to be determined by fitting the above equation to the experimental interactions. The total interaction equation with Debye length as the fitting parameter is named Debye fitting length model (DFLM).

We substituted the measured zeta potential values into Eq. (5.1) and then adjusted the value of the effective Debye length until the theoretical and experimental force curves matched. The final fitting results at different size ratios are shown in Figure 5.2, with the solid lines representing the total interaction force as calculated by the DLFM. 

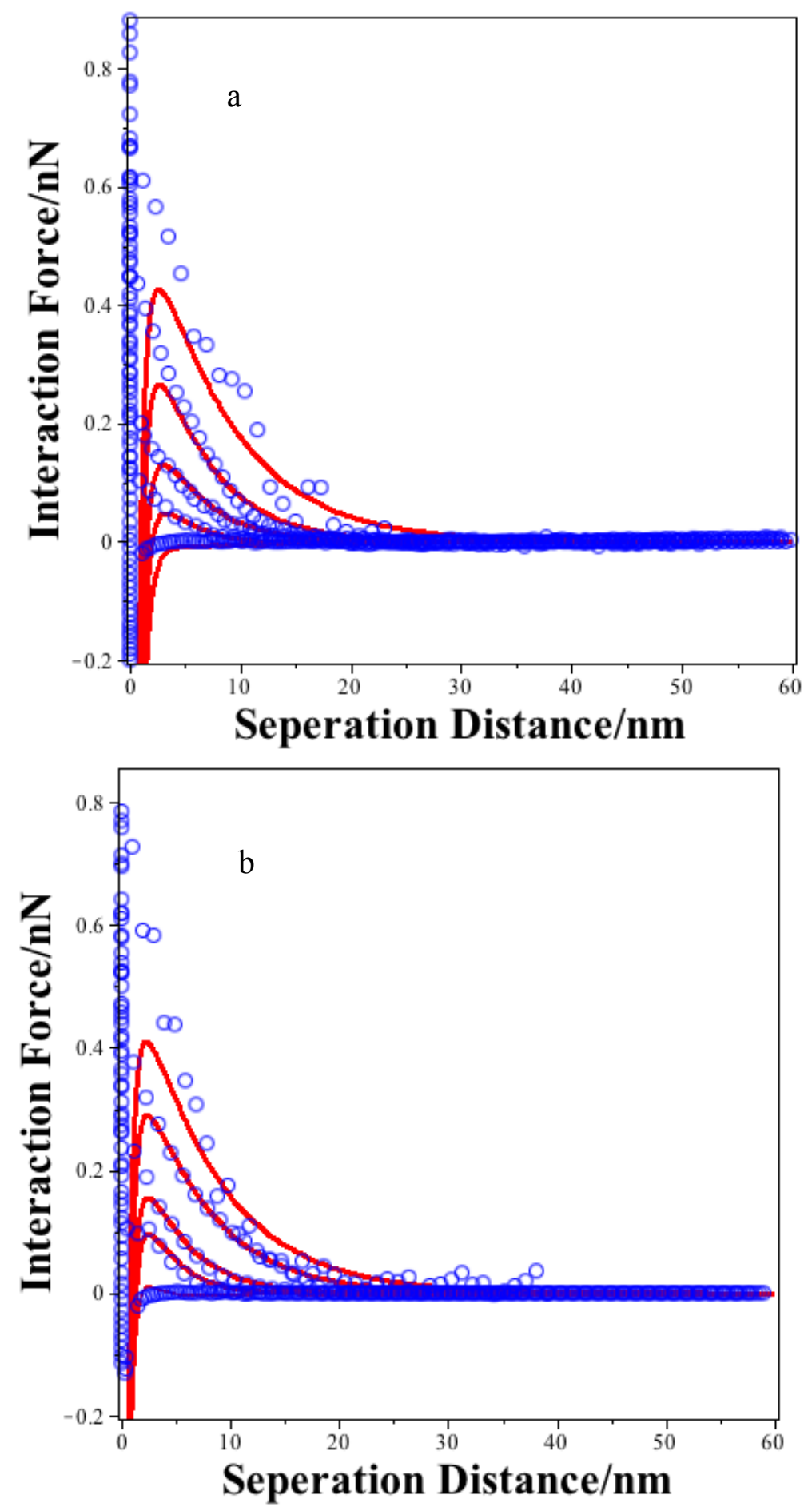

Figure 5.2 Experimental force profiles and fitting results by using DLFM at size ratio of (a) 100; (b) 60. The solid lines represent the total interaction force calculated by the DLFM. 
As seen in Figure 5.2, the interaction calculated by the DLFM shows a good agreement with the experiment data as the nanoparticle volume fraction increases from $10^{-6}$ to $10^{-3}$, indicating the interaction between weakly charged colloidal surfaces surrounded by highly charged nanoparticles is mainly composed of electrostatic repulsion induced by nanoparticle charge layer, van der Waals attraction and depletion force at these concentrations. However, the DLFM tended to underestimate the interaction at separation distance $<10 \mathrm{~nm}$ at volume fraction of $10^{-2}$. It should be noticed that DLFM was developed based on the existence of a "nanoparticle halo" which means nanoparticles are loosely haloing around the colloidal surfaces and would barely impact their surface properties. In other words, the effect of nanoparticle adsorption on the interaction force between colloidal surfaces is ignored in the DLFM. Therefore the failure of DLFM at high nanoparticle concentration is possibly due to the alteration of the van der Waals attraction between colloidal surfaces caused by a strong nanoparticle deposition.

In order to determine if the adsorption of nanoparticles should be expected in the nanoparticle-stabilized binary system at high concentration, the pair-wise potential between a nanoparticle and microsphere is estimated. The electrostatic and van der Waals interactions were calculated using HHF formula and Hamaker's model as mentioned above. The electrostatic potential equation is expressed as:

$$
\begin{gathered}
\mathrm{V}_{\text {macro-nano(D) }}=\frac{1}{4} \varepsilon_{0} \varepsilon_{r} \pi \frac{a_{\text {micro }} a_{\text {nano }}}{a_{\text {micro }}+a_{\text {nano }}}\left(\Psi_{\text {micro }}^{2}+\Psi_{\text {nano }}^{2}\right)\left[\frac{2 \Psi_{\text {micro }} \Psi_{\text {nano }}}{\Psi_{\text {micro }}^{2}+\Psi_{\text {nano }}^{2}} \ln \frac{1+\exp (-\kappa D)}{1-\exp (-\kappa D)}+\right. \\
\ln (1+\exp (-\kappa D)]
\end{gathered}
$$

where $\mathrm{a}_{\text {nano }}$ and $\mathrm{a}_{\text {micro }}$ are the radii of particles, and $\psi_{\text {nano }}$ and $\psi_{\text {micro }}$ are their zeta potentials. The van der Waals potential is given as: 


$$
V_{v d W}(D)=-A \frac{\mathrm{a}_{\text {micro }} a_{\text {nano }}}{\left(\mathrm{a}_{\text {micro }}+a_{\text {nano }}\right) D}
$$

The zeta potentials and radii of nanoparticle and microsphere are listed in Table 5.1.

Table 5.1. Surface potentials for silica microsphere and zirconia nanoparticle at $\mathrm{pH} 1.5$.

\begin{tabular}{ccccc}
\hline $\mathrm{pH}$ & $\mathrm{a}_{\text {nano }}(\mathrm{nm})$ & $\mathrm{a}_{\text {micro }}(\mathrm{nm})$ & $\psi_{\text {nano }}(\mathrm{mV})$ & $\Psi_{\text {micro }}(\mathrm{mV})$ \\
\hline 1.5 & 5 & 500 & 70 & 1 \\
\hline
\end{tabular}

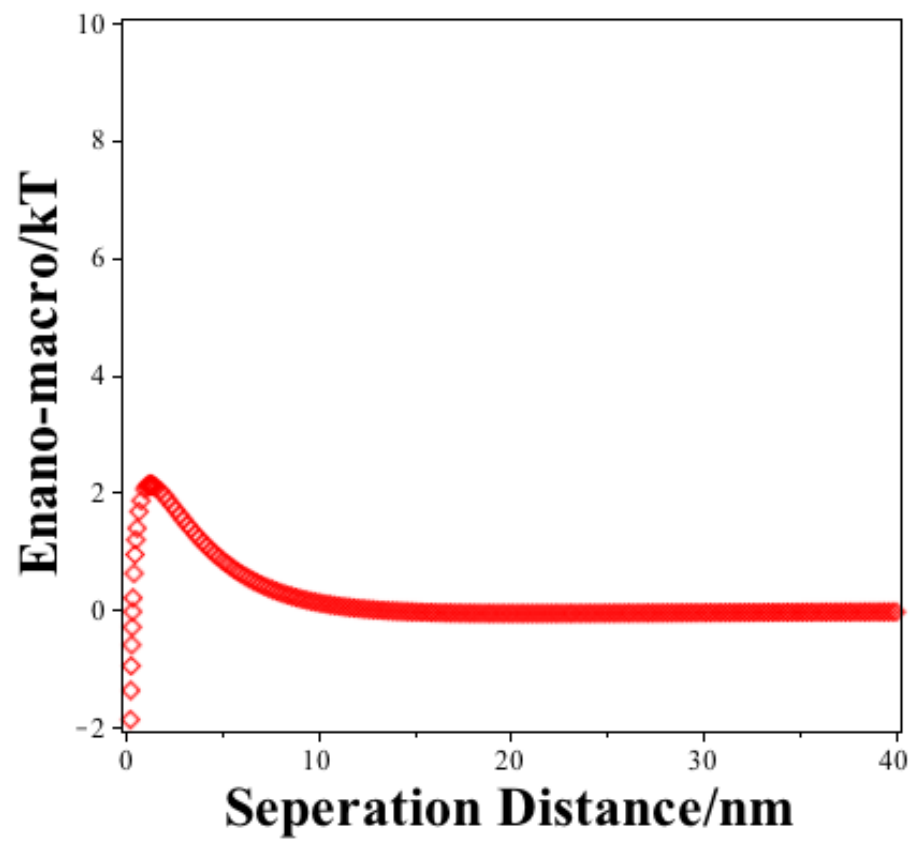

Figure 5.3 The predicted pair potential between a nanoparticle and microsphere.

As shown in Figure 5.3, at $\mathrm{pH}$ of 1.5, the energy barrier between a zirconia nanoparticle and silica microsphere is approximately $2 k T$ at a separation distance of about $2 \mathrm{~nm}$. Under such conditions, it should relatively easy for the nanoparticle to overcome this barrier and deposit directly onto the silica surface, leading to a high degree of nanoparticle adsorption. 

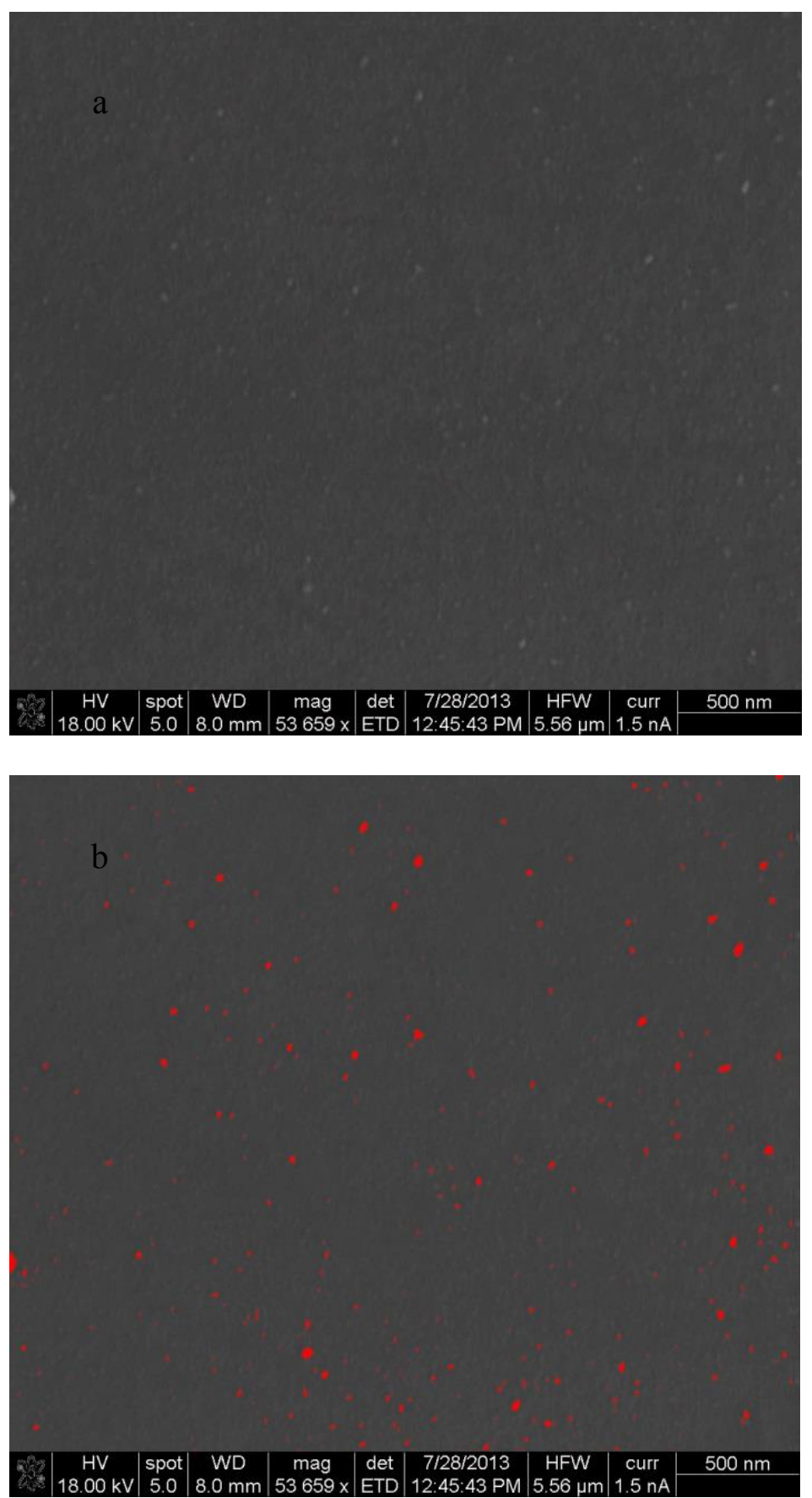

Figure 5.4 SEM image obtained on silica plate that had been immersed in a volume fraction $10^{-4}$ nanoparticle solution for $30 \mathrm{~min}$ at $\mathrm{pH} 1.5$. Zirconia nanoparticles are highlighted in red by ImageJ as seen in b; nanoparticle number and adsorption fraction is estimated by this program as well. 


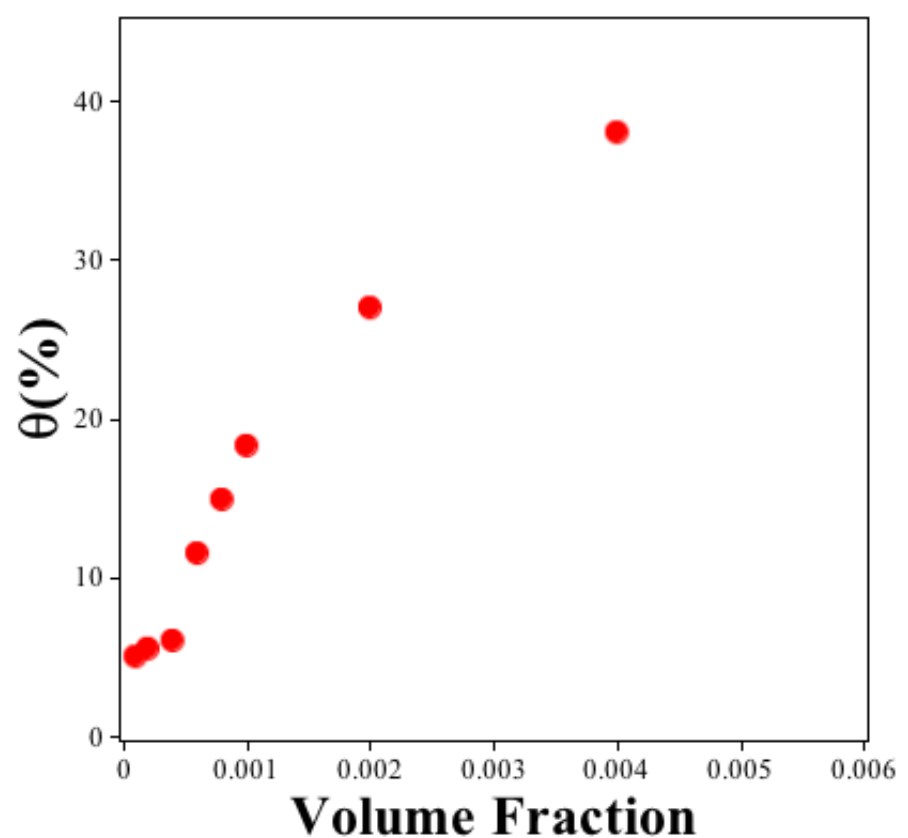

Figure 5.5 Approximate surface coverage fraction $(\theta)$ as the function of nanoparticle concentration at $\mathrm{pH} 1.5$.

To study the degree of nanoparticle adsorption as a function of nanoparticle concentration, the surfaces of silica plates that had been immersed in different nanoparticle suspensions at $\mathrm{pH} 1.5$ were scanned by SEM after 30 min of deposition. Several locations were scanned for each silica plate. The average surface coverage $(\theta \%)$ at different nanoparticle volume fractions was roughly estimated by imageJ (a java-based image processing program) based on these SEM images. For example, Figure 5.4a presents the SEM picture obtained on a silica plate that had been immersed in zirconia nanoparticle solution at volume fraction of $10^{-4}$. Nanoparticles were highlighted by using imageJ (Figure 5.4b), and the calculated coverage fraction was 2.57\%. Surface coverage fraction $(\theta)$ as the function of nanoparticle concentration is plotted in Figure 5.5. The adsorption data was then tested graphically by fitting with various isotherms. It is found 
that the Langmuir adsorption isotherm, which assumes the adsorption process is monolayer over the homogeneous adsorbent surface, is the best description for the nanoparticle adsorption behavior. ${ }^{185}$ The Langmuir isotherm equation is mathematically expressed as follows:

$$
q_{e}=\frac{K q_{m} C_{e}}{1+K C_{e}}
$$

where $q_{m}$ is the maximum adsorption capacity $\left(\mathrm{mg} / \mathrm{g}^{-1}\right) ; q_{e}$ is the amount of nanoparticle adsorbed at equilibrium $\left(\mathrm{mg} / \mathrm{g}^{-1}\right) ; C_{e}$ is the equilibrium concentration of nanoparticle suspension $(\mathrm{mg} / \mathrm{L})$; and $\mathrm{K}$ is the Langmuir constant. We converted $q_{e}$ and $C_{e}$ to surface coverage ratio $\theta_{e}$ and volume fraction ( $\left.C_{v e}\right)$ respectively. Eq.(5.7) is rewritten as Eq.(5.8).

$$
\theta_{e}=\frac{K \theta_{m} C_{v e}}{1+K C_{v e}}
$$

The linear form of Eq.(8) is rearranged as Eq.(5.9).

$$
\frac{C_{v e}}{\theta_{e}}=\frac{1}{K \theta_{m}}+\frac{C_{v e}}{\theta_{m}}
$$

The isotherm parameters obtained from the linear plot of $C_{v e} / \theta_{e}$ verses $C_{v e}$ (Figure 5.6a) are listed in Table 5.2.

Table 5.2. Parameters of the best fit of the data in Figure 5.6.

\begin{tabular}{cccc}
\hline Parameter & $\theta_{\mathrm{m}}(\%)$ & $K$ & $R^{2}$ \\
\hline Value & 59 & 418 & 0.97 \\
\hline
\end{tabular}



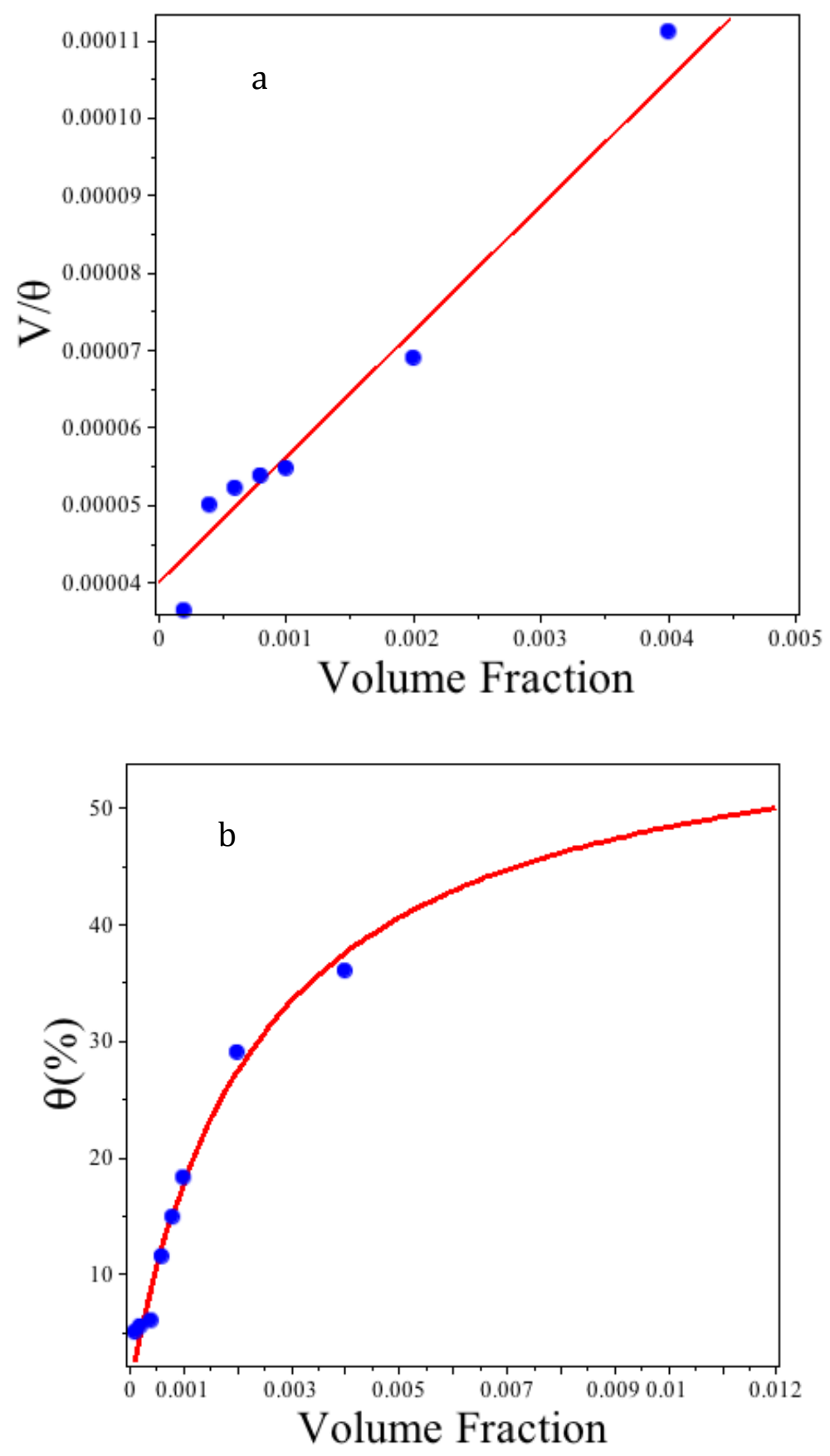

Figure 5.6 (a) Curve fitting of Volume fraction/ surface courage Vs. Volume fraction to Langmuir isotherm; (b) Original form of Langmuir isotherm.

Having obtained the isotherm parameters, the Langmuir isotherm for the zirconiasilica colloidal system in the original form of isotherm equation along with the 
experimental adsorption data is graphed in Figure 5.6b. As seen in Figure 5.6b, nanoparticle deposition was negligible $(\theta<4 \%)$ under volume fraction of $0.5 \times 10^{-3}$. This observation is in accordance with Tohver's finding which showed a significant fraction of nanoparticles remained in bulk solution even at low volume fractions $\left(10^{-6}\right.$ to $\left.10^{-3}\right)$, suggesting zirconia nanoparticles were loosely haloing around colloidal microspheres and would be easily flushed out of system. ${ }^{1}$ However, the adsorption became evident at $10^{-3}$ where there is a surface coverage $\theta \approx 18 \%$. This result is consistent with Ji's adsorption data obtained under similar experimental conditions which showing approximately $20 \%$ surface coverage on silica plate at the same $\mathrm{pH} .{ }^{12}$ The surface coverage gradually grows to about $50 \%$ at $10^{-2}$ as predicated by the Langmuir isotherm, indicating nanoparticles are strongly adsorbed on to the colloidal surfaces rather than haloing around it at higher nanoparticle concentrations. This adsorption study suggests that weakly charged microspheres are stabilized upon the addition of charged zirconia nanoparticles from volume fractions of $10^{-5}$ to $10^{-2}$, but that the stabilization mechanisms are different at varying nanoparticle concentrations: e.g. 1) at low volume fractions (below $10^{-3}$ ), as indicated in previous studies, a non-adsorbing layer of highly charged nanoparticles would form around silica surface, leading to an effective electrostatic repulsive force to overcome van der Waals attraction that drives aggregation; 2) at high volume fraction $\left(\sim 10^{-2}\right)$, nanoparticles are strongly adsorbed onto silica surfaces, inducing an increased repulsion due to an effective increase in the charge on the silica surface; 3) at volume fractions around $10^{-3}$, we hypothesize there is a transition region within which the stabilization can be influenced by both nanoparticle haloing and adsorption. Further study is still needed to understand if this transition region is sensitively dependent on 
nanoparticle size or surface charge, and the starting point of transition is also to be determined.

a

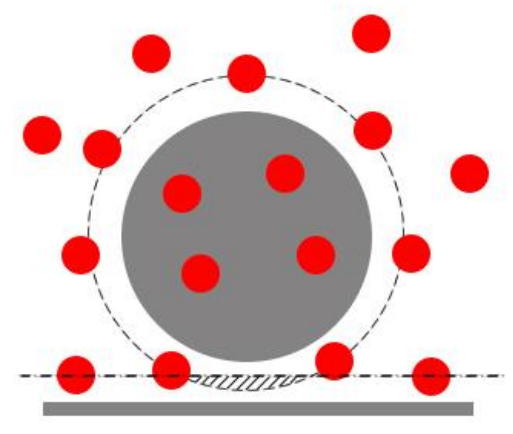

$\mathrm{b}$

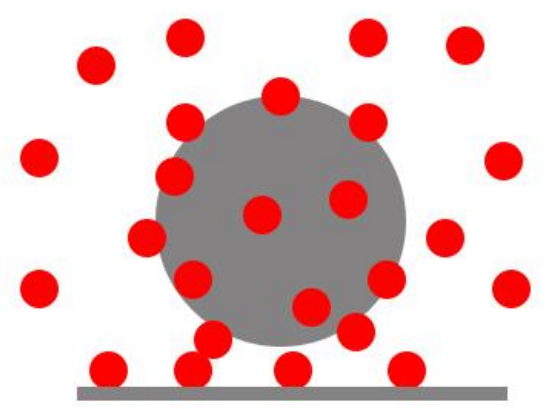

Figure 5.7 Schematic diagram of the interactions between a probe sphere and a flat surface induced by a) nanoparticle halo; b) adsorption.

Under the conditions of nanoparticle haloing, with a weak interaction between the halo and the colloid, the van der Waals attraction between the silica microsphere and plate is barely affected by zirconia nanoparticles as these particles would easily be pushed out of the space between the approaching colloidal surfaces (Figure 5.7a). However, the adsorption study suggests that nanoparticles are strongly absorbed onto the silica surfaces at high nanoparticle concentration. Under these conditions, the adsorbed layer of nanoparticles would alter the physicochemical properties of the silica surfaces (as schematically shown in Figure 5.7b), thus perturbing the van der Waals attraction between them, leading to the van der Waals equation used in DLFM no longer valid. Vold had developed an approach to estimate the van der Waals attraction between spherical particles with an adsorbed layer of thickness $\delta .{ }^{186}$ Based on his formula, at 
$D / \mathrm{a}_{\text {micro }} \ll 1$ and $\delta / \mathrm{a}_{\text {micro }} \ll 1$, the interaction between a microsphere and a plate is derived as: ${ }^{187,188}$

$$
\begin{aligned}
& F_{\text {adsorbed_vdW }}(D)=-\frac{1}{12}\left(\left(A_{w^{\frac{1}{2}}}-A_{z i r^{\frac{1}{2}}}\right)^{2} \frac{a_{\text {micro }}+\delta}{D}+\left(A_{z i r^{\frac{1}{2}}}-A_{s i}\right)^{\frac{1}{2}}\right)^{2} \frac{a_{\text {micro }}}{D+2 \delta}+ \\
& \left.\left(A_{w^{\frac{1}{2}}}-A_{z i r^{\frac{1}{2}}}\right)\left(A_{z i r^{\frac{1}{2}}}-A_{s i} i^{\frac{1}{2}}\right)^{*} \frac{4 a_{m i c r o}\left(a_{m i c r o}+\delta\right)}{\left(2 a_{m i c r o}+\delta\right)(D+\delta)}\right)
\end{aligned}
$$

Where $A_{w}, A_{z i r}$, and $A_{s i}$ are the Hamaker constants of water, the zirconia nanoparticles and silica, respectively. $A_{w}=3.7 \times 10^{-20} \mathrm{~J}, A_{z i \mathrm{r}}=6 \times 10^{-20} \mathrm{~J},{ }^{189} A_{s i}=0.8 \times 10^{-20} \mathrm{~J}$. The thickness of adsorbed layer is taken as $9 \mathrm{~nm}$, which is the average diameter of the zirconia nanoparticles.

The total interaction equation under high-adsorption conditions is then rewritten as Eq.(5.11):

$$
F_{\text {adsorbed_total }}(D)=F_{\text {adsorbed_vdW }}+F_{\text {electro }}+F_{\text {depletion }}
$$

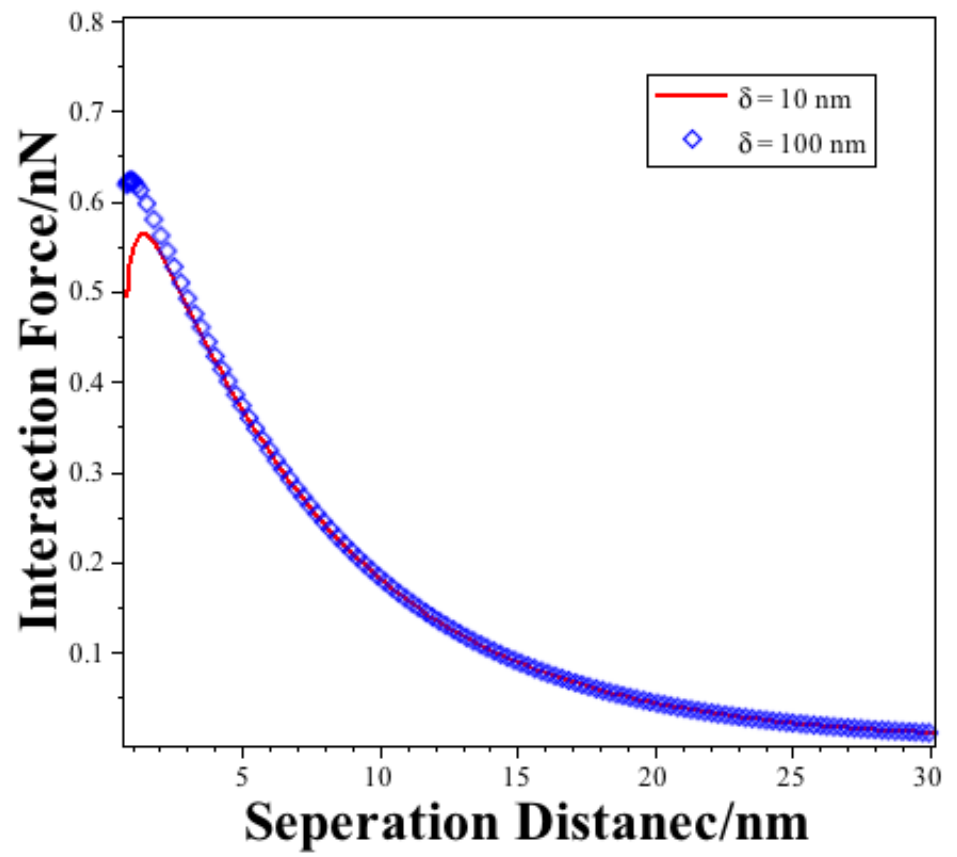

Figure 5.8 Force curves calculated at different thickness of adsorb layer. 
As suggested by the Langmuir isotherm, the adsorption pattern of zirconia nanoparticle onto silica surface is single layered, meaning the maximum thickness of adsorbed layer is about the diameter of zirconia nanoparticle $(10 \mathrm{~nm})$. Thus the actual adsorbed thickness $\delta$ at varying nanoparticle concentrations ranges from 0 to $10 \mathrm{~nm}$. Since $\delta$ is required for van der Waals force calculation, the effect of $\delta$ on total interaction force is firstly discussed in the modified DLFM. At volume fraction of $10^{-3}$, the total interaction forces have been estimated based on different $\delta$ (Shown in Figure 5.8). It can be seen clearly in Figure 5.8 that the effect of $\delta$ on total interaction force is negligible; merely difference was observed between different thicknesses ( $10 \mathrm{~nm}$ and $100 \mathrm{~nm}$ ) even when $\delta$ is taken as ten times of the nanoparticle diameter. This result is very reasonable. At high degree of surface coverage of nanoparticles, the van der Waals forces is mainly affected by the change of surface properties (such as Hamaker constants for different species), which are rarely related to the thickness of adsorbent layer. Therefore, thickness is taken as $10 \mathrm{~nm}$ in our calculation for simplicity.

Subsequently, the total interaction force between a silica microsphere and plate in suspension with zirconia volume fractions of $10^{-2}$ and $10^{-4}$ is recalculated by using the revised total interaction equation (5.11); the fitting curves calculated using the original DLFM are also graphed for comparison (dash lines in Figure 5.9). 

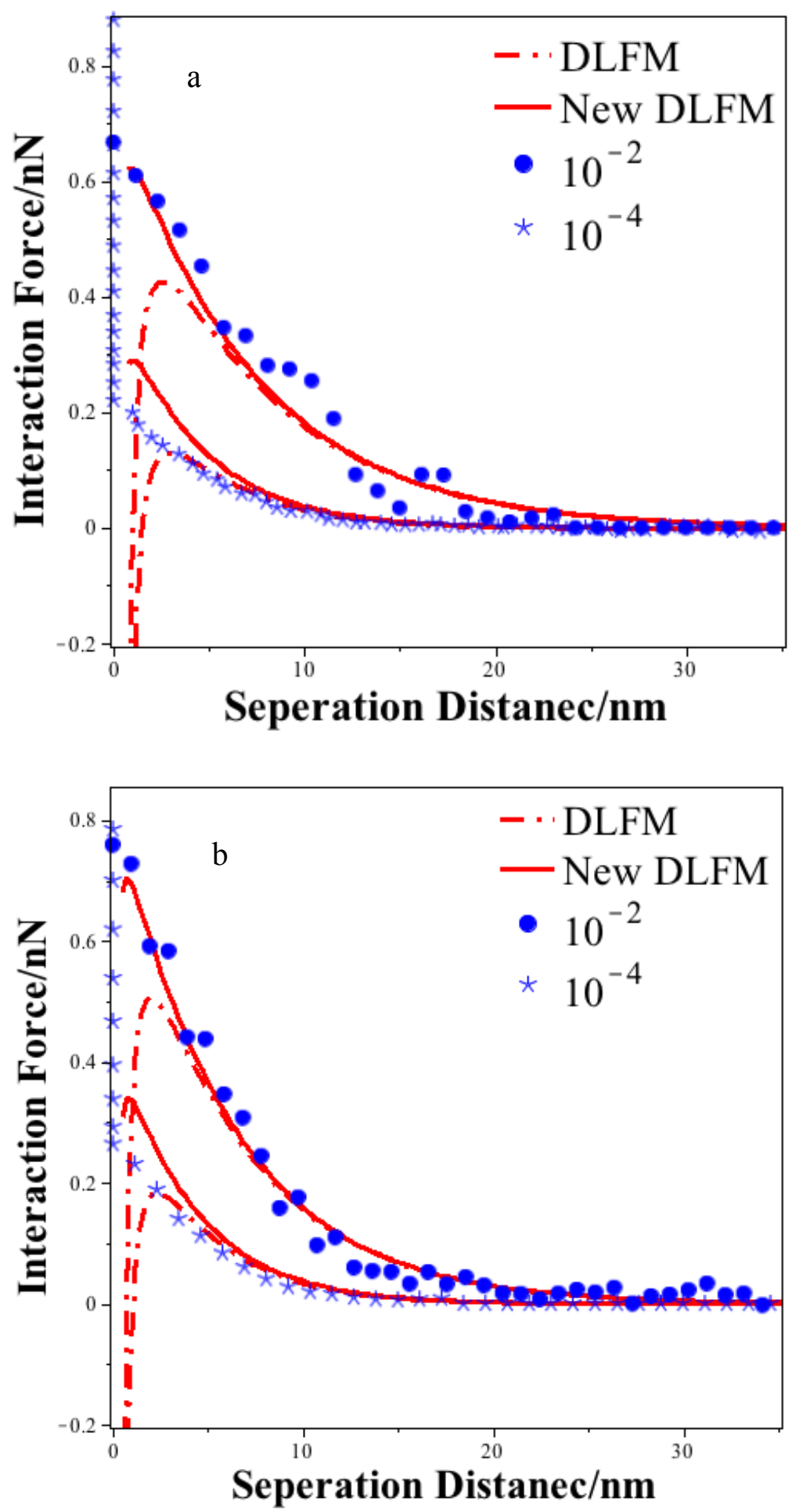

Figure 5.9 Force curves measured at volume fraction $10^{-2}$ and $10^{-4}$, and fitting results using the modified DLFM at size ratio of a) 100 ; b) 60 . Sold line presents modified DLFM fitting curve; dash line stands for the original DLFM fitting curve. 
As seen in Figure 5.9, the interaction estimated by the modified DLFM has a much better fit to experimental data at a nanoparticle volume fraction of $10^{-2}$. This result proves that at high nanoparticle concentrations nanoparticles are strongly adsorbed onto colloidal surfaces. This adsorbed layer weakens the van der Waals force between colloids, so that the total interaction is strengthened as a result, leading to the theoretical interaction being able to match the experimentally obtained force curve. On the other hand, the overestimation of the modified DLFM curve at a nanoparticle volume fraction of $10^{-4}$ indicates the interaction calculated by Eq.(5.11) is inadequate to estimate the interaction between colloidal surfaces at low nanoparticle concentrations, suggesting strong adsorption is unexpected at these concentrations and that nanoparticles are most likely haloing around the silica spheres with a non-zero separation distance between them. The success in matching experimental force curves by choosing the appropriate van der Waals formulas in the DLFM is consistent with our conclusion mentioned above that: "nanoparticle haloing" is the primary stabilization mechanism at low nanoparticle concentrations while colloidal suspensions are stabilized primarily by "nanoparticle adsorption" at high concentrations. Through these measurements, the DLFM has been improved to estimate the interactions in a nanoparticle-stabilized system over a comprehensive range of nanoparticle concentrations. 


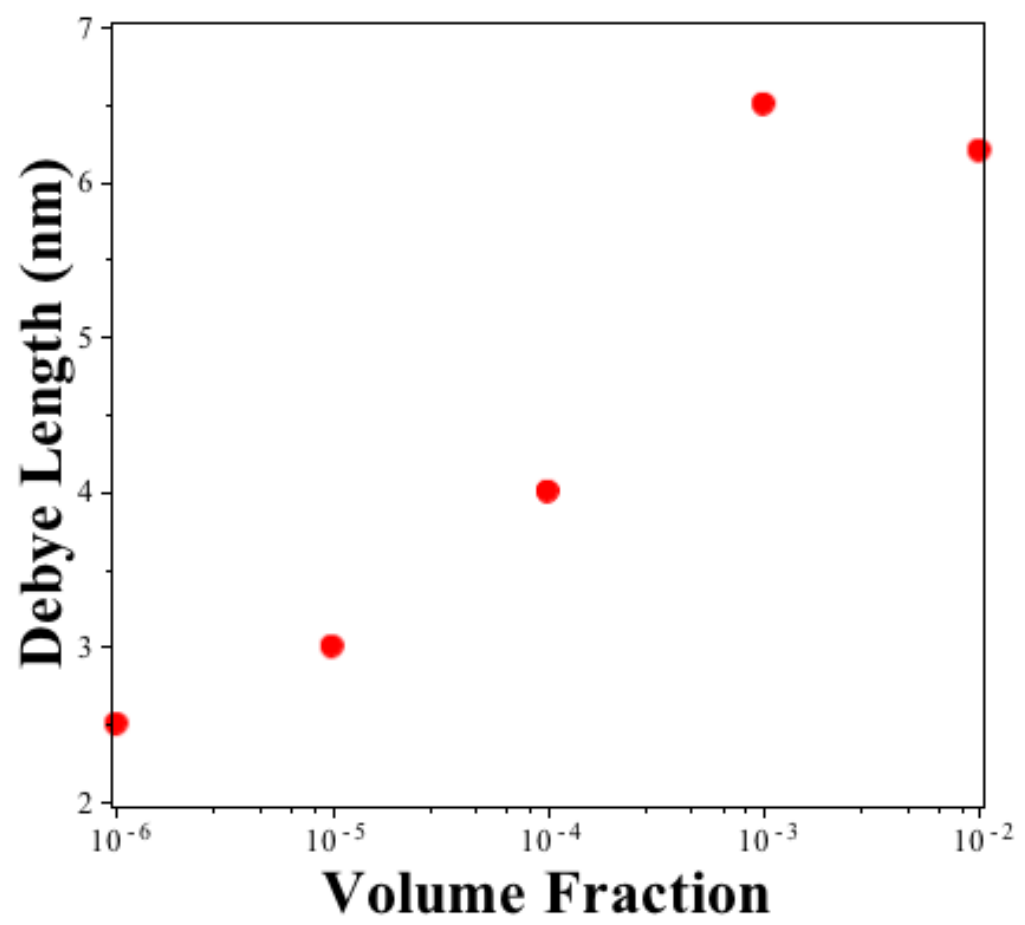

Figure 5.10 Fitted Debye length as the function of nanoparticle volume fraction at size ratio of 100 .

As mentioned in the previous chapter, from volume fraction of $10^{-6}$ to $10^{-3}$, the Debye length obtained from the fitting process increases with the increasing nanoparticle concentrations, meaning the distance between colloidal surface and charge layer is broadened as the number of surrounded nanoparticle increasing. The fitted Debye length as a function of nanoparticle volume fraction is summarized in Figure 5.10. The Debye lengths at volume fraction of $10^{-6}$ to $10^{-3}$ were calculated by using the original DLFM, and the fitted Debye length at volume fraction $10^{-2}$ is estimated by using the new DLFM. As seen in Figure 5.10 at volume fraction of $10^{-2}$, the Debye length obtained from modified DLFM is $6.2 \mathrm{~nm}$, which is slightly smaller than that at $10^{-3}(6.5 \mathrm{~nm})$. This result is expected. At high nanoparticle adsorption the Debye length should be smaller than that under nanoparticle halo condition, because there is no separation between colloidal 
microsphere and adsorbed nanoparticle layer. But it still lager than $4 \mathrm{~nm}$-Debye length estimated at volume fraction of $10^{-4}$, suggesting despite approximately $50 \%$ surface adsorption at volume fraction of $10^{-2}$, there are still a portion of nanoparticles haloing around silica surface which enlarges the value of Debye length.

Despite nanoparticles being capable of stabilizing colloidal suspensions based on either nanoparticle halo or adsorption mechanism, it's of great importance to distinguish their working conditions. For example, if nanoparticle concentration is controlled within nanoparticle-halo zone, the stabilization will not rely on adsorption, making it suitable in applications where using an adsorbed species may hinder reactivity or availability of the colloidal surface such as in colloidal surface functionalization and ceramics processing. Further study is needed to elucidate if this transition between "halo" and "adsorption" is sensitive to the suspension properties, including nanoparticle size and surface charge. Additionally, the starting point of transition needs to be determined.

\subsection{Conclusion}

In summary, we have performed a comprehensive experimental investigation on the interaction between neutral colloidal surfaces in highly charged nanoparticle aqueous solutions. It is found that the silica-zirconia binary suspension system could be stabilized by highly charged nanoparticles at volume fractions ranging from $10^{-5}$ to $10^{-2}$. A subsequent adsorption isotherm study showed that nanoparticle deposition was negligible below a volume fraction of $0.5 \times 10^{-3}$, but became significant from $10^{-3}$ to $10^{-2}$. This result suggests that "nanoparticle haloing" is responsible for nanoparticle-regulated stabilization at low nanoparticle volume fractions (below $10^{-3}$ ), while "nanoparticle adsorption" is in

charge of the stabilization at high volume fractions $\left(\sim 10^{-2}\right)$. We hypothesize that there is a 
transition region around a nanoparticle volume fraction of $10^{-3}$, within which the stabilization mechanism can be influenced by both nanoparticle haloing and adsorption.

The DLFM that was developed initially to estimate the interaction between colloidal particles in the presence of charged nanoparticles has been further extended to relatively high nanoparticle concentration by revising the term of van der Waals to adopt a strong adsorptive condition.

Our study suggests when using highly charged nanoparticles to stabilize colloidal suspensions, the two fundamental mechanisms of nanoparticle haloing and adsorption are not mutually exclusive. They work continuously to regulate the stability of colloidal suspensions over increasing nanoparticle concentrations. Depending on the ultimate application of the colloids, the primary mechanism can be controlled by simply tuning the nanoparticle concentrations. Further studies are needed to more fully elucidate the role of nanoparticle size and charge on the transition from stabilization by nanoparticle haloing to nanoparticle adsorption. 


\section{CHAPTER 6}

\section{CONCLUSIONS AND FUTURE DIRECTIONS}

\subsection{Conclusion}

In this study, in order to elucidate the stabilization mechanism of nanoparticleregulated complex fluid, interparticle interactions between silica colloidal surfaces surrounded by charged zirconia nanoparticles have been thoroughly investigated in both experimental and theoretical methods. The specific deliverables from this work are:

1. Interaction forces between a weakly charged silica sphere and plate in highly charged zirconia nanoparticle solutions at varying concentrations and size asymmetries has been measured by using CP-AFM technique. Zeta potential results show that highly charged zirconia nanoparticles are able to enhance the stability of silica colloidal suspensions when the nanoparticle volume fraction is above $10^{-5}$. The follow-up force measurements confirm that the interaction between silica surfaces is dominated by attraction at low nanoparticle concentration, and shifts to repulsion as zirconia volume fraction increased to $10^{-5}$. These results demonstrate that highly charged nanoparticles would gather around silica surfaces, leading to an effective charging layer surrounding negligible charged silica. Once the effective electrostatic repulsion induced by nanoparticle charge layer is sufficient to overcome the van der Waals attraction between colloidal surfaces at higher nanoparticle concentration, the colloidal system will be stabilized. 
2. A Debye length fitting model (DLFM) has been developed to estimate the interaction force between neutral colloidal microspheres that are surrounded by highly charged nanoparticle halos. The DLFM suggests: 1) the interaction between microspheres in the presence of nanoparticles is mainly composed of a van der Waals attraction and an electrostatic repulsion, depletion force is negligible when nanoparticle volume fraction is lower than $\left.10^{-3} ; 2\right)$ the effect of nanoparticle adsorption on the van der Waals attraction between colloidal surfaces is negligible within volume fractions of $10^{-4}$ and $10^{-6}$. Additionally, the fitting result of effective Debye length indicates that the gap between colloidal surface and surrounding charge layer increases with the increasing nanoparticle concentrations. Since this stabilization method of nanoparticle halo does not rely on adsorption, it is specifically suitable in applications where using adsorbed species may hinder reactivity or availability of the surface such as in colloidal surface functionalization and ceramics processing.

3. Nanoparticle haloing and adsorption two stabilization mechanisms are not mutually exclusive when using charged nanoparticles to regulate the stability of colloidal suspensions; they work continuously over the increasing nanoparticle concentrations. The experimental force measurements showed that the silica-zirconia binary suspension system could be stabilized by highly charged nanoparticles at volume fractions ranging from $10^{-5}$ to $10^{-2}$. A subsequent adsorption isotherm study showed that nanoparticle deposition was negligible below a volume fraction of $10^{-3}$, but became significant from $10^{-3}$ to $10^{-2}$. This result suggests that "nanoparticle haloing" is responsible for nanoparticle-regulated stabilization at low nanoparticle volume fractions (below $10^{-3}$ ), while "nanoparticle adsorption" is in charge of the stabilization at high volume fractions 
$\left(\sim 10^{-2}\right)$. We hypothesize that there is a transition region around $10^{-3}$ where the stabilization mechanism can be influenced by both nanoparticle haloing and adsorption. Depending on the ultimately application of the colloids, the primary mechanism can be controlled by simply tuning the nanoparticle properties such as concentrations, sizes or charges. Additionally, The DLFM that was developed initially to estimate the interaction between colloidal particles in low concentrations of charged nanoparticles has been further extended to relatively high nanoparticle concentrations by revising the term of van der Waals to adopt a strong adsorptive condition.

\subsection{Future Directions}

Several important directions of future research can be identified.

\subsubsection{Determine the Critical Conditions of Nanoparticle Halo and Adsorption}

The study in Chapter 5 shows that the additional nanoparticles tend to halo around colloidal microsphere at low nanoparticle concentrations, and are more likely to deposit onto colloidal surfaces at high concentrations. However, the critical condition where the fundamental mechanism transit from nanoparticle haloing to adsorption is still to be determined. Our further interaction study between colloidal surfaces in the presence of charged nanoparticles will be specific to the nanoparticle volume fraction within $10^{-3}$ and $10^{-2}$, to observe when the transition occurs and how the nanoparticle behaves during the transition process. Besides, the effects of other solution conditions like $\mathrm{pH}$, ionic strength and nanoparticle size on the transition region are to be studied as well.

\subsubsection{Introduce nanoparticle halo mechanism to industry process}

After the investigation of critical conditions of nanoparticle haloing and adsorption, one should be able to control the primary mechanism to be either nanoparticle 
haloing or adsorption by tuning nanoparticle properties. If under the condition of haloing, since the stabilization method through nanoparticles does not rely on adsorption, it is specific suitable in applications where using adsorbed species may hinder reactivity or availability of the surface such as in colloidal surface functionalization and ceramics processing. Take the manufacture of Dye-sensitized solar cell (DSSC) for example: a porous layer of titanium dioxide particles made of titanium dioxide paste serves as an anode in DSSC. If a certain species of conductive nanoparticles is utilized to enhance the stability of this gel paste, the $\mathrm{TiO} 2$ surfaces would not only be free to adsorb light and provide travel paths for electron, but also be improved in conductivity due to the additional nanoparticles. Future study could be focused on finding out appropriate species of nanoparticles which is able to well stabilize the $\mathrm{TiO} 2$ paste while improving the efficiency of DSSC. 


\section{REFERENCES}

1. Lewis, J. A. Colloidal Processing of Ceramics. J. Am. Ceram. Soc. 2000, 83, 2341-2359.

2. Smay, J. E.; Gratson, G. M.; Shepherd, R. F.; Cesarano, J., III; Lewis, J. A. Directed Colloidal Assembly of 3D Periodic Structures. Adv. Mater. 2002, 14, $1279-1283$.

3. Martinez, C. J.; Lewis, J. A. Shape Evolution and Stress Development During Latex-Silica Film Formation. Langmuir 2002, 18, 4689-4698.

4. Joannopoulos, J. D.; Villeneuve, P. R.; Fans, S. Photonic Crystals: Putting a New Twist on Light. Nature 1997, 386, 143-149.

5. Yablonovitch, E. Inhibited Spontaneous Emission in Solid-State Physics and Electronics. Phys. Rev. Lett. 1987, 58, 2059-2062.

6. Braun, P. V.; Wiltzius, P. Electrochemically grown photonic crystals. Nature 1999, 402, 603-604.

7. Pan, G.; Kesavamoorthy, R.; Asher, S. A. Optically Nonlinear Bragg Diffracting Nanosecond Optical Switches. Phys. Rev. Lett. 1997, 78, 3860-3863.

8. Johnson, S. A.; Oliver, P. J.; Mallouk, T. E. Ordered Mesoporous Polymers of Tunable Pore Size from Colloidal Silica Templates. Science 1999, 283, 963-965.

9. Burmeister, F.; Schafle, C.; Matthes, T. Colloid Monolayers as Versatile Lithographic Masks. Langmuir 1997, 13, 2983-2987. 
10. Muller, R. H. Colloidal Carriers for Controlled Drug Delivery and Targeting, Modification, Characterization, and In Vivo Distribution; CRC Press: Boca Raton, FL, 1991.

11. Lange, F. F.; Powder Processing Science and Technology for Increased Reliability. J. Am. Ceram. Soc. 1989, 72, 3-15.

12. Aksay, I.A.; Microstructure Control through Colloidal Consolidation. pp.94-104 in Advances in Ceramics, Vol. 9, Forming of Ceramics Edited by J. A. Mangels and G. L. Messing. American Ceramic Society, Columbus, OH, 1984.

13. Guo, J.; Lewis, J. A. Aggregation Effects on Compressive Flow Properties and Drying Behavior of Colloidal Silica Suspensions. J. Am. Ceram. Soc. 1999, 82, $2345-2358$.

14. Israelachvili, J. N., Intermolecular and surface forces. London: Academic Press; 1992.

15. Hunter, R. J. Foundation of Colloid Science, $2^{\text {nd }}$ ed., Oxford University Press, Oxford, 2001.

16. Derjaguin, B. V.; Landau, L. D. Theory of the stability of strong charged lyophobic sols and the adhesion od strongly charged particles in solutions of electrolytes. Acta. Physicochim URSS 1941, 14, 633.

17. Verwey, E. J.; Overbeek, J. T. Theory of the stability of lyophobic colloids 1948, Elsevier Publishing Company, Inc., Amsterdam.

18. Bowen, W.R., Jenner, F. Theoretical descriptions of membrane filtration of colloids and fine particles - an assessment and review. Adv. Colloid. Interface Sci. 1995, 56, 141-200. 
19. Dzyaloshinskii, I.E.; Lifshitz, E.M.; Pitaevski, L.P. The general theory of van der Waals forces. Adv. Phys. 1961, 10, 165-209.

20. Hough, D.B.; White, L.R. The calculation of Hamaker constants from Lifshitz theory with applications to wetting phenomena. Adv. Colloid Interface Sci. 1980, $14,3-41$.

21. Ninham, B.W.; Parsegian, V.A. van der Waals forces - special characteristics in lipid-water systems and a general method of calculation based on Lifshitz theory. Biophys J. 1970, 10, 646.

22. London, F. Zur Theorie und Systematik der Molekularkraefte. Z Phys. 1930, 63, $245-279$.

23. Wang, S.C. The mutual influence between hydrogen atoms. Phys $Z$ 1927, 28, $663-666$.

24. Margenau, H. The role of quadrupole forces in van der Waals attractions. Phys. Rev. 1931, 38, 747-756.

25. Casimir, H.B.G.; Polder, D. The influence of retardation on the London-van der Waals forces. Phys Rev. 1948, 73, 360-372.

26. Hamaker, H.C. The London-van der Waals attraction between spherical particles. Physica 1937, 4, 1058-1072.

27. de Boer, J.H. The influence of van der Waals' forces and primary bonds on binding energy, strength and orientation, with special reference to some artificial resins. Trans. Faraday Soc. 1936, 32, 10-37.

28. Lifshitz, E.M. The theory of molecular attractive forces between solids. Sov Phys JETP USSR 1956, 2, 73-83. 
29. Gregory, J. The calculation of Hamaker constants. Adv. Colloid Interface. Sci. 1970, 2, 396-417.

30. Horn, R.G.; Israelachvili, J.N. Direct measurement of structural forces between 2 surfaces in a non-polar liquid. J. Chem. Phys. 1981, 75, 1400-1411.

31. Israelachvili, J.N. Calculation of van der Waals dispersion forces between macroscopic bodies. Proc. R. Soc. Lond A 1972, 331, 39.

32. Mahanty, J.; Ninham, B.W. Dispersion forces 1976. Academic Press, New York.

33. Prieve, D.C.; Russel, W.B. Simplified predictions of Hamaker constants from Lifshitz theory. J. Colloid Interface Sci. 1988, 125, 1-13.

34. Russel, W.B.; Saville, D.A.; Schowalter, W.R. Colloidal dispersions 1989. Cambridge University Press, Cambridge.

35. Stern, O. The theory of the electrolytic double shift. Z Elektrochem 1924, 30, $508-516$.

36. Derjaguin, B.V. Friction and adhesion IV. The theory of adhesion of small particles. $\quad$ Kolloid Z 1934, 69, 155-164.

37. Bell, G.M.; Levine, S.; McCartney, L.N. Approximate methods of determining the double-layer free energy of interaction between two charged colloidal spheres. J Colloid Interface Sci. 1970, 33, 335-359.

38. Gregory, J. Interaction of unequal double layers at constant charge. J. Colloid Interface Sci. 1975, 51, 44-51.

39. Hogg, R.I.; Healey, T.W.; Fuerstenau, D.W. Mutual coagulation of colloidal dispersions. Trans. Faraday Soc. 1966, 62, 1638-1651. 
40. Wiese, G.R.; Healy, T.W. Effect of particle size on colloid stability. Trans. Faraday Soc. 1970, 66, 490-500.

41. Ohshima, H.; Kondo, T. Comparison of three models on double layer interaction. J. Colloid Interface Sci. 1988, 126, 382-383.

42. Kar, G.; Chander, S.; Mika, T.S. The potential energy of interaction between dissimilar electrical double layers. J. Colloid Interface Sci. 1973, 44, 347-355.

43. McCartney, L.N.; Levine, S. An improvement on Derjaguin's expression at small potentials for the double-layer interaction energy of two spherical colloidal particlesrticles. J. Colloid Interface Sci. 1969, 30, 345-354.

44. Kuwabara, S. The forces experienced by randomly distributed parallel circular cylinders or spheres in a viscous flow at small Reynolds number. J. Phys Soc. Jpn. 1959, 14, 527-532.

45. Bowen, W.R.; Jenner, F.; Dynamic ultrafiltration model for charged colloidal dispersions: a Wigner-Seitz cell approach. Chem. Eng. Sci. 1995, 50, 1707.

46. Bowen, W.R.; Williams, P.M. J. Colloid Interface Sci. 1996, 184, 241.

47. Reiner, E.S.; Radke, C.J. Electrostatic interactions in colloidal suspensions tests of pairwise additivity. AIChE J. 1991, 37, 805-824.

48. Liang, Y.; Hilal, N.; Langston, P.; Starov, V. Interaction forces between colloidal particles in liquid: Theory and experiment. Adv. Colloid Interface Sci. 2007, 134, 151-166.

49. Evans, D. F.; Wennerström, H. The colloidal domain: Where Physics, Chemistry, Biology and Technology meet. VCH publishers, 1994. 
50. Berg, C. J. An Introduction to Interfaces and Colloids: The Bridge to Nanoscience. World Scientific Publishing Co. Ptc. Ltd, 2010.

51. Israelachvili, J. Intermolecular and Surfaces Forces. 2nd. Edition. Academic Press, 1991.

52. Xie, H.; Yu W. A review on nanofluids: Preparation, stability mechanisms and applications. J. Nanomaterials 2012, doi: 10.1155/2012/435873.

53. Sader, J. E.; Carnie, S. L.; Chan, D. Y. J. Colloid Interface Sci. 1995. 171, 46.

54. Cesarano, J. III; Aksay, I. A.; Bleier, A. Stability of Aqueous a $-\mathrm{Al}_{2} \mathrm{O}_{3}$ Suspensions with Poly(methacrylic acid) Polyelectrolyte. J. Am. Ceram. Soc. 1988, $71,250-255$.

55. Biggs, S.; Healy, T. W. Electrosteric Stabilization of Colloidal Zirconia with Low Molecular Weight Polyacrylic Acid. J. Chem. Soc. Faraday Trans. 1994, 90, $3415-3421$.

56. Rojas, D. J.; Claesson, P. M.; Muller, D.; Neuman, R. The Effect of Salt Concentration on Adsorption of Low-Charge-Density Polyelectrolytes and Interactions between Polyelectrolyte-Coated Surfaces. J. Colloid Interface Sci. 1998, 205, 77-78.

57. Marra. J.; Hair, M. Forces between Two Poly(2-vinyl pyridine)-Covered Surfaces as a Function of Ionic Strength and Polymer Charge. J. Phys. Chem. 1988, 92, $6044-6051$.

58. Hackley, V. Colloidal Processing of $\mathrm{Si}_{3} \mathrm{~N}_{4}$ with PAA: I, Adsorption and Electrostatic Interactions. J. Am. Ceram. Soc. 1997, 80, 2315-2325. 
59. Lewis. J. A. Colloidal Processing of Ceramics. J. Am. Ceram. Sci. 2000, 83, 2341-2359.

60. Dumont, F.; Ameryckx, G.; Watillon, A. Colloids Surf. 1990, 51, 171.

61. Luckham, P. F.; Tadros, T. F.; Hart, C. A.; Vincent, B. Colloids Surf. 1980, 1, 281.

62. Hansen F. K.; Matijevic, E. J. Chem. Soc., Faraday Trans. 1980, 76, 1240.

63. Furusawa, K.; Anzai, C. Colloids Surf. 1992, 63, 103.

64. James, R. O.; Homola, A.; Healy, T. W. J. Chem. Soc., Faraday Trans. 1977, 73, 1436.

65. Kitano, H.; Iwai, S.; Ise, N.; Okubo, T. J. Am. Chem. Soc. 1987, 109, 6641.

66. Harley, S.; Thompson, D. W.; Vincent, B. Colloids Surf. 1992, 62, 163.

67. Islam, A. M.; Chowdhry, B. Z.; Snowden, M. J. Adv. Colloid Interface Sci. 1995, $62,109$.

68. Jenkins, P.; Snowden, M. Adv. Colloid Interface Sci. 1996, 68, 57.

69. Sharma, A.; Tan, S. N.; Walz, J. Y. J. Colloid Interface Sci. 1997, 190, 392.

70. Asakura, S., Oosawa, F. On Interaction between two bodies immersed in a solution of macromolecules. J. Chem. Phys., 1954, 22, 1255-1256.

71. Asakura, S., Oosawa, F. Interaction between particles suspended in solutions of macromolecules. J. Polym. Sci., 1958, 33, 183-192.

72. Walz, J. Y., Sharma, A. Effectof long range interactions on the depletion force between colloidal particles, J.Colloid Interface Sci.1994, 168,485-496.

73. Walz, J. Y., Effect of poly dispersity on the depletion interaction between colloidal particles, J.Colloid Interface Sci. 1996, 178, 505-513. 
74. Sharma, A., Walz, J. Y., Direct measurement of the depletion interaction in a charged colloidal dispersion, J.Chem.Soc.Faraday Trans.1996, 92, 4997-5004.

75. Mao, y., Cates, M. E., Theory of the depletion force due to rod like polymers, J.Chem.Phys. 1997, 160, 3721-3729.

76. Piech, M., Walz, J. Y., Depletion interactions produced by nonadsorbing charged and uncharged spheroids, J.Colloid Interface Sci. 2000, 232, 86-101.

77. Helden, L., Koenderink, G. H., Leiderer, P., Bechinger, C., Depletion potentials induced by charged colloidal rods, Langmuir 2004, 20, 5662-5665.

78. Verma, R., Crocker, J. C., Lubensky, T.C., Yodh, A.G. Entropic colloidal interactions in concentrated DNA solutions, Phys.Rev.Lett.1998, 81, 4004-4007.

79. Odiachi, P. C., Prieve, D. C., Effect of added salt on the depletion attraction caused by non-adsorbing clay particles, Colloids Surf.,A. 1999, 146, 315-328.

80. Piech, M., Walz, J.Y., Analytical expressions for calculating the depletion interaction produced by charged spheres and spheroids, Langmuir 2000, 16, 7895-7899.

81. Piech, M., Walz, J.Y., Effect of poly dispersity and charge heterogeneity on the depletion interaction in colloidal systems, J.Colloid Interface Sci.2000, 225, 134146.

82. Piech, M., Walz, J.Y., Direct measurement of depletion and structural forces in poly disperse, charged systems, J.Colloid Interface Sci. 2002, 253, 117-129.

83. Piech, M., Weronski, P., Xu, X., Walz, J.Y. Prediction and measurement of the interparticle depletion interaction next to a flat wall, J.Colloid Interface Sci. 2002, $247,327-341$. 
84. Weronski, P., Walz, J.Y., An approximate method for calculating depletion and structural interactions between colloidal particles, J.Colloid Interface Sci. 2003, 263, 327-332.

85. González-Mozuelos, P., Medina-Noyola, M., Electro static trapping of a colloidal monolayer near a charged wall, J.Chem.Phys. 1991, 94, 1480-1486.

86. González-Mozuelos, P., Alejandre, J., Rogers-Young approximation for the concentration profile of a colloidal suspension in front of a highly repulsive wall, J.Chem.Phys. 1996, 105, 5949-5955.

87. Trokhymchuk, A., Henderson, D., Nikolov, A., Wasan, D.T., Computer modeling of ionic micelle structuring in the films, J.Phys.Chem.B 2003, 107, 3927-3937.

88. VanWinkle, V., Murray, C.A., Layering in colloidal fluids near a smooth repulsive wall, J.Chem.Phys. 1988, 89, 3885-3891.

89. Murray, C.A., Sprenger, W.O., Wenk, R.A., Microscopic structure and dynamic sofa dense fluid near a smooth wall video microscopy of colloidal spheres, J.Phys.:Condens.Matter 1990, 2, 385-SA388.

90. Murray, C.A., Grier, D.G., Video microscopy of mono disperse colloidal systems, Annu.Rev.Phys.Chem. 1996, 47, 421-462.

91. VonKlitzing, R., Thormann, E., Nylander, T., Langevin, D., Stubenrauch, C., Confinement of linear polymers, surfactants, and particles between interfaces, Adv. Colloid Interface 2010, 155, 19-31.

92. Zeng, Y., vonKlitzing, R., Structuring of colloidal suspensions confined between a silica microsphere and an air bubble, Soft Matter 2011, 7, 5329-5338. 
93. Tulpar, A., Walz, J.Y., Simultaneous measurement of structural and hydrodynamic forces between colloidal surfaces in complex fluids, Colloids Surf., A 2007, 268-280.

94. Tulpar, A., VanTassel, P.R., Walz, J.Y., Structuring of macroions confined between like-charged surfaces, Langmuir 2006, 22, 2876-2883.

95. Fazelabdolabadi, B., Walz, J.Y., VanTassel, P.R. Influence of charged nanoparticles on colloidal forces : a molecular simulation study, J.Phys.Chem.B 2009, 113, 13860-13865.

96. Drelich, J., Long, J., Xu, Z., Masliyah, J., Nalaskowski, J., Beauchamp, R., Liu, Y., AFM colloidal forces measured between microscopic probes and flat substrates in nanoparticle suspensions, J.Colloid Interface Sci. 2006, 301, 511522.

97. Mckee, C.T., Walz, J.Y., Interaction forces between colloidal particles in a solution of like-charged, adsorbing nanoparticles. J. Colloid Interface Sci., 2012, $356,72-80$.

98. Tohver, V.; Smay, J. E.; Braem, A.; Braun, P. V.; Lewis, J. A. Nanoparticle Halos: A New Colloid Stabilization Mechanism. Proc. Natl. Acad. Sci. U.S.A. 2001, 98(16), 8950-8954.

99. Tohver, V.; Chan, A.; Sakurada, O.; Lewis, J. A. Nanoparticle Engineering of Complex Fluid Behavior. Langmuir 2001, 17, 8414-8421.

100. Chan, A. T.; Lewis, J. A. Size Ratio Effects on Interparticle Interactions and Phase Behavior of Microsphere-Nanoparticle Mixtures. Langmuir 2008, 24, 11399-11405. 
101. Barr, S. A.; Luijten, E. Effective Interactions in Mixtures of Silica Microspheres and Polystyrene Nanoparticles. Langmuir 2006, 22, 7152-7155.

102. Chan, A. T.; Lewis, J. A. Electrostatically Tuned Interactions in Silica Microsphere-Polystyrene Nanoparticle Mixtures. Langmuir 2005, 21, 8576-8679.

103. Kong, D. Y.; Yang, Y.; Wei, S.; Wang, H. B.; Cheng, B. J. Dispersion Behavior and Stabilization Mechanism of Alumina Powders in Silica Sol. Mater. Lett. 2004, 58, 3503-3508.

104. Xing, X.; Sun, G.; Li, Z.; Ngai, T. Stabilization of Colloidal Suspensions: Competing Effects of Nanoparticle Halos and Depletion Mechanism. Langmuir 2012, 28(46), 16022-16028.

105. Zhang, F.; Long, G. G.; Jemian, P. R.; Ilavsky, J. Quantitative Measurement of Nanoparticle Halo Formation around Colloidal Microspheres in Binary Mixtures. Langmuir 2008, 24(13), 6504.

106. Liu, J.; Luijten, E. Colloidal Stabilization via Nanoparticle Halo Formation. Phys. Rev. Lett. 2005, 72, 061401.

107. Liu, J.; Luijten, E. Stabilization of Colloidal Suspensions by Means of Highly Charged Nanoparticles. Phys. Rev. Lett. 2004, 93, 247802.

108. Viveros-Méndez, P. X.; Méndez-Alcaraz, J. M.; González-Mozuelos, P. Formation and Structure of Colloidal Halos in Two-dimensional Suspensions of Paramagnetic Particles. J. Chem. Phys. 2012, 136(16), 164902.

109. Trulsson, M.; Jonssona, B.; Labbezc, C. On the Origin of the Halo Sabilization. Phys. Chem. Chem. Phys., 2013, 15(2), 541. 
110. Huang, H.; Ruckenstein, E. Decoration of Microparticles by Highly Charged Nanoparticles. J. Phys. Chem. B,. 2013, 117 (20), 6318-6322.

111. Huang, H.; Ruckenstein, E. Repulsive Force Between Two Microparticles Decorated with Highly Charged Nanoparticles. Colloids Surf., A 2013, 436(5), $862-867$.

112. Hong, X. T.; Willing, G. A. Transition Force Measurement between Two Negligibly Charged Surfaces: A New Perspective on Nanoparticle Halos. Langmuir 2009, 9, 4929.

113. Dylla, R.; Wong, L.; Miller, P.; Feit, M. Charged micelle halo mechanism for agglomeration reduction in metal oxide particle based polishing slurries. Colloid Surfaces A. 2014, 447, 32-43.

114. Derjaguin, B. V., Abrikkssova II, Lifshitz, E. M. Direct measurement of molecular attraction between solids separated by a narrow gap. Quart Rev 1956, $10,295$.

115. Derjaguin, B. V., Titijevskaia, A. S., Abricossova II, Malkina, A. D., Investigations of the forces of interaction of surfaces in different media and their application to the problem of colloid stability. Discuss Faraday Soc 1954, 18, 2441.

116. Israelachvili, J. N., Adams, G. E., Measurement of forces between 2 mica surfaces in aqueous-electrolyte solutions in the range 0-100 nm. J. Chem. Soc. Faraday Trans. I 1978, 74, 975.

117. Pashley, R. M. Hydration forces between mica surfaces in aqueous electrolyte solutions. J. Colloid Interface Sci. 1981, 80, 153-62. 
118. Horn, R. G., Smith, D. T., Haller, W. Surface forces and viscosity of water measured between silica sheets. Chem. Phys. Lett. 1989, 162, 404-8.

119. Hirz, S. J., Homola, A. M., Hadziioannou, G., Frank, C. W. Effect of substrate on shearing properties of ultrathin polymer-films. Langmuir 1992, 8, 328-33.

120. LeNeveu, D.M., Rand, R.P., Parsegian, V.A. Nature 1976, 259, 601.

121. Parsegian,V.A., Rand, R.P., Fuller, N.L., Rau, D.C. Meth. Enzymol. 1986, 127, 400.

122. Parsegian, V.A., Fuller, N., Rand, R.P. Proc. Natl. Acad. Sci. U.S.A. 1979, 76, 2750.

123. Prieve, D.C. Adv. Colloid Interf. Sci. 1999, 82, 93.

124. Binnig Q, Quate CF, Gerber C. Phys Rev Lett 1986, 56, 930.

125. Bowen WR, Hilal N, Lovitt RW, Williams PM. J Membr. Sci. 1996, 110, 229.

126. Ducker WA, Senden TJ, Pashley RM. Nature 1991, 353, 2239.

127. Ducker, W. A.; Senden, T. J. Measurement of Forces in Liquids Using a Force Microscope. Langmuir 1992, 8, 1831.

128. Vigil, G., Xu, Z., Steinberg, S., Interactions of silica surfaces. J Colloid Interface Sci 1994, 165, 367-85.

129. Bowen, W.R., Hilal, N., Jain, M., Lovitt, R.W., Sharif, A.O., Wright, C.J., The effects of electrostatic interactions on the rejection of colloids by membrane pores visualization and quantification. Chem Eng Sci, 1999, 54, 369 - 375.

130. Bowen, W.R., Hilal, N., Lovitt, R.W. and Williams, P.M., Visualization of an ultrafiltration membrane by non-contact atomic force microscopy at single pore resolution. Journal of Membrane Science 1996, 110, 229 - 232. 
131. Bowen, W.R., Hilal, N., Lovitt, R.W. and Williams, P.M., Atomic force microscope studies of membranes: Surface pore structures of Cyclopore and Anopore membranes, Journal of Membrane Science 1996, 110, 233 - 238.

132. Bowen, W.R., Hilal, N., Lovitt, R.W. and Williams, P.M., Atomic force microscope studies of membranes: Surface pore structures of Diaflo ultrafiltration membranes, Colloid and Interface Science 1996, 180, 350 - 359.

133. Bowen, W.R., Hilal, N., Lovitt, R.W. and Wright, C.J., Atomic force microscope studies of membrane surfaces. Surface Chemistry and Electrochemistry of Membrane Surfaces 1999, 79, 1-37.

134. Butt, H.J. Biophys. J. 1991, 60, 1438.

135. Hilal, N. and Kochkodan, V., Surface modified micro filtration membranes with molecularly recognizing properties, Journal of Membrane Science 2003, 213, 97 $-113$.

136. Hilal, N., Kochkodan, V., Nigmatullin, R., Goncharuk, V. and Al-Khatib, L. Lipase-immobilized biocatalytic membranes for enzymatic esterification: comparison of various approaches to membrane preparation, Journal of Membrane Science 2005, 268, 198 - 207.

137. Bowen, W.R., Hilal, N., Lovitt, R.W. and Wright. Direct measurement of interactions between adsorbed protein layers using an atomic force microscope, Coll Int. Sci. 1998, 197, $348-352$.

138. Karaman, M.E., Meagher, L., Pashley, R.M. Langmuir 1993, 9, 1220.

139. Huntington, S.T., Hartley, P.G., Katsifolis, J. Ultramicroscopy 2003, 94, 283.

140. Clark, S.C., Walz, J.Y., Ducker, W.A. Langmuir 2004, 20, 7616. 
141. Park Atomic Force Microscopy. https://www.parkafm.com/

142. Khatiwada, D.; Lamichhane, S. K. A Brief overview of AFM force distance spectroscopy. The Himalayan Physics 2011, 2, 80.

143. Cappella, B.; Dietler, G. Force-distance curves by atomic force microscopy. Dietler/Surface Science Reports 1999, 34.

144. Rosoff(ed). M. Nano surface Chemistry, Marcel Dekker Inc NY, USA 2002.

145. Maugis, D. J. Colloid Interf. Sci 1992. 150, 243-269.

146. Carpick, R. W.; Ogletree, D. F.; Salmeron, M. J. Colloid Interf. Sci. 1999, 211, $395-400$.

147. John Ralston, Pure and applied chemistry 2005, 77, 2149-2170.

148. Grierson, D. S.; Flater, E. E.; Carpick, R. W. Accounting for the JKR-DMT transition in adhesion and friction measurements with atomic force microscopy. $J$. Adhesion Sci. Technol. 2005, 19, 291-311.

149. Greenwood, R; Kendall, K (1999). J. Eur. Ceram. Soc. 1999, 19, 479-488.

150. Hanaor, D.A.H.; Michelazzi, M.; Leonelli, C.; Sorrell, C.C. The effects of carboxylic acids on the aqueous dispersion and electrophoretic deposition of ZrO2. J. Eur. Ceram. Soc. 2005, 32, 235-244.

151. Reimer, L. Scanning Electron Microscopy; Physics of Image Formation and Microanalysis. 2nd. edition. Springer Verlag, 1998.

152. Vernon-Parry. K. D. In: III-Vs Review 2003, 13, 40-44.

153. Putnis, A. Introduction to Mineral Sciences. Cambridge University Press, 1992.

154. Kingery, W. D. Firing-The Proof Test for Ceramic Processing, Ceramic Processing Before Firing. Wiley, New York, 1978, 291-305. 
155. Bowen, H. K. Basic Research Needs on High-Temperature Ceramics for Energy Applications. J. Mater. Sci. Eng. 1980, 44, 1.

156. Rhodes, W. H. Agglomeration and Particle Size Effects on Sintering of YttriaStabilized Zirconia. J. Am. Ceram. Soc. 1981, 64, $19-22$.

157. Lange, F. F. Processing Related Fracture Origins: I, II, and III. J. Am. Ceram. Soc. 1983, $65,396-408$.

158. Stober, W.; Fink, A.; Bohn, E. Controlled Growth of Monodisperse Silica Spheres in the Micron Size Range. J. Colloid Interface Sci. 1968, 26, 62- 69.

159. Matijevic, E. Monodispersed Metal (Hydrous) Oxides-A Fascinating Field of Colloid Science. Acc. Chem. Res. 1981, 14, 22-29.

160. Barringer, E. A.; Bowen, H. K. Formation, Packing, and Sintering of Monodisperse TiO 2 Powders. J. Am. Ceram. Soc. 1982, 65, 199-201.

161. Brinker, C. J.; Clark, D. E.; Ulrich, D. R. Materials Research Society Symposium Proceedings, Better Ceramics Through Chemistry. Elsevier, New York, 1984.

162. Liufu, S.; Xiao, H.; Li, Y. Adsorption of Poly(acrylic acid) onto the Surface of Titanium Dioxide and the Colloidal Stability of Aqueous Suspension. J. Colloid Interface Sci. 2005, 281, 155-163.

163. Shen, G.; Chen, F.; Zhou, K.; Yun, J. Dispersion of Nanosized Aqueous Suspensions of Barium Titanate with Ammonium Polyacrylate. J. Colloid Interface Sci. 2004, 275, 158-164.

164. Singh, B.; Bhattacharjee, S.; Besra, L.; Sengupta, D. K. Evaluation of Dispersibility of Aqueous Alumina Suspension in Presence of Darvan C. Ceram. Int. 2004, 30, 939-946. 
165. Scheer, E. N., Schweizer, K. S. , J. Chem. Phys., 2008, 128, 164905.

166. Mitlin, V., J. Colloid Interface Sci. 2004, 285, 879-880.

167. Gao, H., Xiao, C., Ke, H., Physics Letters A, 2007, 362, 234-238.

168. Neu, B., Meiselman, H. J., Biochimica et Biophysica Acta (BBA), 2006, 1760, $1772-1779$.

169. Xiao, C., Wylie, J., Physics Letters A, 2006, 357, 245-248.

170. Suematsu, N. J., Nishimura, S., Yamaguchi, T., Langmuir, 2008, 24, 2960-2962.

171. Perera, L., Essmann, U., Berkowitz, M. L., Langmuir, 1996, 12, 2625-2629.

172. Craig, V. S. J., Ninham, B. W., Pashley, R. M., Langmuir, 1999, 15, 1562-1569.

173. Gu, C., Kirkpatrick, A., Ray, C., Guo, S., Akhremitchev, B. B. J. Phys. Chem. C., 2008, 112, 5085-5092.

174. Ghzaoui, A. E., J. Appl. Phys., 1999, 86, 5894-5897.

175. Larson, I., Drummond, C. J., Chan, D. Y. C., Grieser, F. J. Phys. Chem., 1995, 99, 2114-2118.

176. Li, Y. Q., Tao, N. J., Pan, J., Garcia, A. A., Lindsay, S. M. , Langmuir, 1993, 9, $637-641$.

177. Renger, C., Kuschel, P., Kristoffersson, A., Clauss, B., Oppermann, W., Sigmund, W., Phys. Chem. Chem. Phys., 2004, 6, 14671-474.

178. McNamee, C. E., Tsujii, Y., Ohshima, H., Matsumoto, M. Langmuir, 2004, 20, 1953-1962.

179. Drelich, J., Long, J., Xu, Z., Masliyah, J., White, C. L. J. Colloid Interface Sci. 2006, 303, 627-638. 
180. Drelich, J., Long, J., Xu, Z., Masliyah, J., Nalaskowski, J., Beauchamp, R., Liu, Y. J. Colloid Interface Sci. 2006, 301, 511-522.

181. Ji, S.; Herman, D.; Walz, J. Manipulating microparticle interactions using highly charged nanoparticles. Colloids Surf., A, 2012, 396(20), 51-62.

182. Chatterjee, A. P.; Schweizer, K. S. Microscopic Theory of Polymer-mediated Interactions between Spherical Particles. J. Chem. Phys. 1998, 109, 10464-10476.

183. Chatterjee, A. P.; Schweizer, K. S. Correlation Effects in Dilute Particle-polymer Mixtures. J. Chem. Phys. 1998, 109, 10477-10488.

184. Chan, D.; Pashley, R.; White, L. A simple algorithm for the calculation of the electrostatic repulsion between identical charged surfaces in electrolyte. J. Colloid Interface Sci. 1980, 77, 283-285.

185. Hoa, Y.; Chiub, W.; Wanga, C. Regression Analysis for the Sorption Isotherms of Basic Dyes on Sugarcane Dust. Bioresour. Technol. 2005, 96, 1285-1291.

186. Vold, M. J. The Effect of Adsorption on the van der Waals Interaction of Spherical Colloidal Particles. J. Colloid Sci. 1961, 16,1-12.

187. Wang, Q.; Luo, G.; Niu, J.; Jin, J.; Wang, H. The Surface Potential of a Coal-oil Mixture and Control of Their Stability. Energy Sources, Part A 2013, Vol. 35, Iss. 21.

188. Borgnino, L. Experimental determination of the colloidal stability of Fe (III)montmorillonite: Effects of organic matter, ionic strength and $\mathrm{pH}$ conditions. Colloids and Surfaces A 2013, 423, 178-187. 
189. Prica, M.; Biggs, S.; Grieser, F.; Healy, T. W. Effect of Calcination Temperature on the Electrokinetic Properties of Colloidal Zirconia. Colloids Surf., A. 1996, 119, 205-213. 


\title{
CURRICULUM VITAE
}

\section{Qingwen He}

\author{
Department of Chemical Engineering \\ University of Louisville \\ Ernst Hall, Room 206, Louisville, KY 40292, USA
}

Tel: $1-502-424-3217$

Email: qingwen.he@louisville.edu

\section{Education}

Ph.D. in Chemical Engineering University of Louisville, Louisville, KY, US, GPA

3.78/4 (Aug.2009-Aug.2014)

B.S. in Chemical Engineering Beijing University of Chemical Tech., Beijing, China, GPA 3.49/4 (Aug.2005-May.2009)

\section{Experience}

Graduate Research (Aug.2009-May.2014)

University of Louisville

\section{Bio-materials Characterization}

- Tracked the morphological impact of respiratory syncytial virus (RSV) infection on

Hep-2 cells by using Atomic force microscopy (AFM).

- Studied the effect of size diversity of gold nanoparticles on targeting cellular components for biomedical applications by microscopic studies (AFM+SEM). 


\section{Stabilization of Colloidal Suspension by Nanoparticles}

- Directly measured the interaction force between silica colloidal surfaces in the presence of charged nanoparticles using Colloidal Probe technique.

- Developed a debye length fitting model (DLFM) to estimate the interaction between colloidal microspheres in the presence of charged nanoparticles.

- Proposed the reversibility of stabilization and accessibility of colloidal surfaces required for chemical reaction can be controlled by tuning nanoparticle concentration when using charged nanoparticles to regulate stability of colloidal suspension.

\section{UV Polymerization of Oligothiophenes in Dye-sensitized Solar Cells (DSSC)}

- Infiltrated polythiophene into $\mathrm{TiO} 2$ nano-porous film through the process of UV polymerization, providing a cheap and easy method to improve infiltration of polymers into nano-porous film during the manufacture of DSSC.

- Extensive experience in characterization of materials using AFM, SEM/EDX, Raman spectroscopy, FTIR, UV-Vis spectroscopy, Fluorescence spectroscopy.

- Trained new users in the proper use of AFM.

- Mentored a high school student in his science project on DSSC manufacture and testing.

Teaching Assistant (Aug.2009-May.2014) University of Louisville

- Undergraduate courses of Materials Science, Introduction to Chemistry Lab

- Graduate courses of Heat Transfer and Advanced Reactor Design

Professional Affiliation American Institute of Chemical Engineers

\section{Publications}


Q. He, X. Hong, G. Willing, Tuning The Stabilization Mechanism of Nanoparticleregulated Complex Fluids, Langmuir 2014, submitted.

C. Boyoglu, Q. He, G. Willing, et al., Microscopic Studies of Various Sizes of Gold Nanoparticles and Their Cellular Localizations, ISRN Nanotechnology 2013. (DOI: $10.1155 / 2013 / 123838)$

P.M. Tiwari, Q. He, G. Willing, et al., Atomic Force Microscopic Investigation of Respiratory Syncytial Virus Infection in HEp-2 Cells, Journal of Microscopy 2014, 253, $31-41$.

\section{Presentations}

"Interaction Forces between Colloidal Particles within a Nanoparticle Suspension."

(Oral) American Institute of Chemical Engineers Annual Meeting, Pittsburgh, PA,

October 2012 